SAND-89-7018

DE92 0.18938

SAND89-7018

Unlimited Release

Printed July 1992

Conceptual, Experimental and Computational

Approaches to Support Performance Assessment of

Hydrology and Chemical Transport at

Yucca Mountain

T. N. Narasimhan and J. S. Y. Wang

Earth Sciences Division

Lawrence Berkeley Laboratory

University of California

Berkeley, CA 94720

for

Saridia National Laboratories

P.O. Box 5800

Albuquerque, NM 87185

Under Sandia Contract: $75-4347$

Sandia Contract Monitor

P. G. Kaplan

System Performance-Assessment Division 


\begin{abstract}
The authors of this repon have been participating in the Sandia National Laboratory's hy'drologic performance assessment of the Yucca Mountain, Nevada, since 1983. This participation has resulted in the conceptual analysis of several important hydrological issues relating to the movement of water through the partially saturated fractured tuff rock system constituting the Yucca Mountain hydrologic system. Meanwhile, the Congress of the United States chose Yucca Mourtain for characterization as part of the program to provide a repository for high-level nuclear wastes. As a consequence, Yucca Mountain is now the focus of attention aimed at demonstrating to the satisfaction of the Nuclear Regulatory Commission whether the site will be safe to operate. At this time of transition in the evolution of the Yucca Mountain Site Characterization Project (YMP), this repon is an effort to identify the important scientific and engineering issues that need resolution to suppont the licensing application. The scope of this work is restricted to the unsaturated zone at Yucca Mountain and to technical questions about hydrology and chemical transport. The issues defined here are not to be confused with the elaborate hierarchy of issues that forms the framework of the U. S. Depanment of Energy plans for characterizing the site (DOE, 1989 !.

The overall task of hydrologic performance assessment involves issues related to hydrology, geochemistry, and energy transport in a highly heterogeneous natural geologic system which will be perturbed in a major way by the disposal activity. Therefore, a rational evaluation of the performance assessment issues must be based on an integrated appreciation of the aforesaid interacting processes. Accordingly, a hierarchical approach is taken in this report, proceeding from the statement of the broad features of the site that make it the site for intensive studies and the rationale for disposal strategy, through the statement of the fundamental questions that need to be answered, to the identification of the issues that need resolution. Having identified the questions and issues, the repon then outlines the tasks to be undertaken to resolve the issues

Available hydrogeological and geochemical evidence suggests that the unsaturated zone at Yucea Mountain has many desirable features conducive to retarding the migration of contaminants from the repository to the accessible environment. However, the strong perturbations (excavations and heat input) that will be introduced by the proposed repository as well as the time
\end{abstract}


scale of prediction $(10,000 \mathrm{yr})$ pose special challenges to the venture of hydrological performance assessment. The potential impact of this challenge is mitigated by another favorable feature of the Yucca Mountain, namely, amenability to long term monitoring and retrievability of the wastes. Indeed, retrievability provides an open endedness, an aspect that is indispensable in designing complex engineering systerns.

The report consists essentially of two parts. The first part deals with the definition of issues summarized above. The second part summarizes the findings of the authors between 1983 and 1989 under the activities of the former Nevada Nuclear Waste Storage Investigation (NNWSI) and the current YMP. In brief, the important infererices drawn are as follows:

- Under ambient conditions, moisture movement in the vadose zone at Yucca Mountain is dominated by the tuff matrix.

- Hydraulic parameters, such as conductivity, saturation and effective surface area, are dictated by the nature of the roughness of the fractures.

- Because of the dominance of capillary forces in the extremely fine pores, strong infiltration pulses tend to be damped out by near-surface welded and nonwelded units.

- If the Ghost Dance Fault is assumed to be made up of highly permeable open fractures and hence is treatable as a seepage face, then the small eastward tilt of the stratigraphic units (approximately $6^{\circ}$ ) is not sufficient to greatly enhance lateral flow over verical flow.

- Hydraulic parameters, such as permeability, saturation, air entry pressure, are mutually correlated at least in a crude way and hence judicious constraints will have to be placed in choosing hydraulic parameters for modeling exercises.

- Rough-walled fractures can be quantified using fractal theory and parameters of fractal theory can be advantageously related to parameters of geostatistics. 
The work of preparing this report was conducted under WBS Element 1.2.1.4.4.1. 


\section{Acknowledgments}

We would like to thank Scott Sinnock of Sandia National Laboratories for initiating the idea of taking an overall integrated look at the issues of performance assessment, and Paul Kaplan, also of SNL, for continued discussions throughout the course of this study. Isaac Winograd of the U.S. Geological Survey provided many valuable insights, especially on the evolution of ideas pertaining to the choice of Yucca Mountain as a potential site for the disposal of high level wastes. Discussions with our colleagues Karsten Pruess, Chin Fu Tsang, Yvonne Tsang and John Apps helped greatly in formalizing our thoughts on the critical issues. The critical technical comments by Ralston Bamard and Paul Kaplan helped considerably in improving the quality of the final version of this report. The work was supported by the Sandia National Laboratories for the Yucca Mountain Site Characterization Project of the U.S. Deparment of Energy. 


\section{Table of Contents}

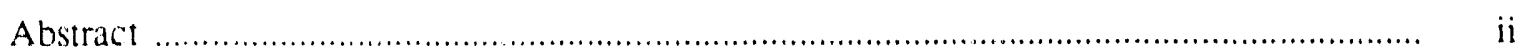

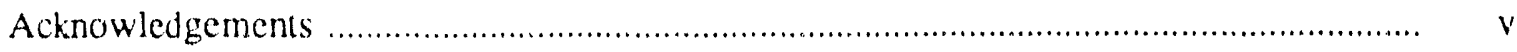

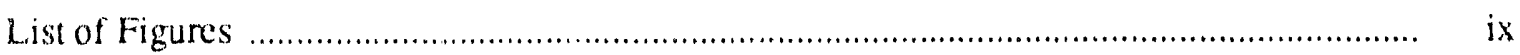

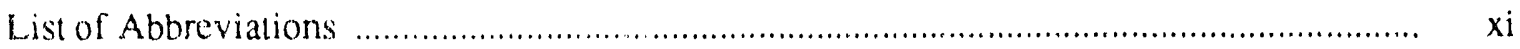

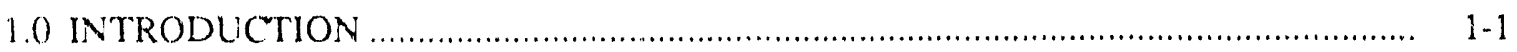

1.1 LBL Participation .....................................................................................

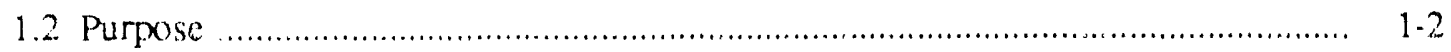

2.0 KEY ISSUES: UNSATURATED ZONE HYDROLOGY ….................................. 2-1

2.1 Repository Requirements ...................................................................... $2-1$

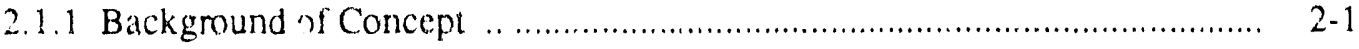

2.1.2 Factors Favoring Yucca Mountain .................................................... $2-2$

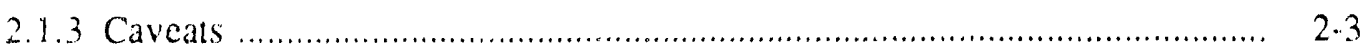

2.1.4 Nature of Technical Challenge .......................................................... 2-3

2.1 .5 A Rationale for Solution ................................................................. $2-5$

2.1.6 Accessible Environment ..................................................................... 2-6

2.1.7 A Measure of Performance ............................................................... 2-6

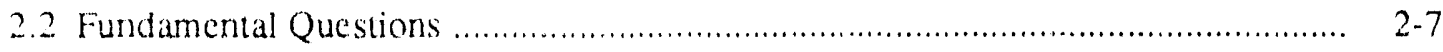

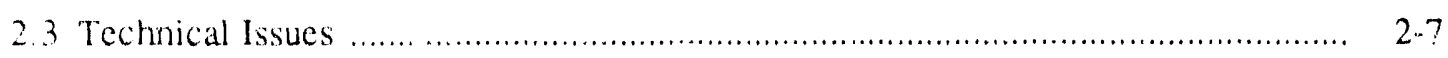

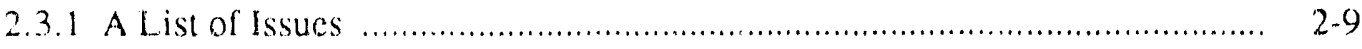

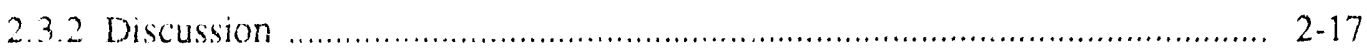

3.) IMPORTANT TASKS FOR THE RESOLUTION OF HYDROLOGIC

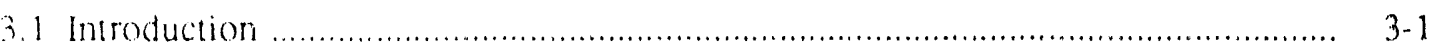

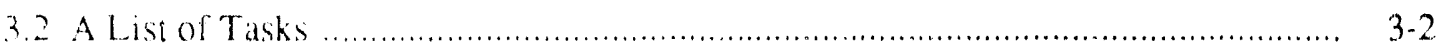

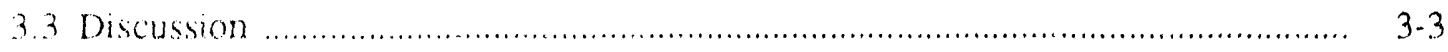

3.3.1 Long-Tem Evolution of the Yucca Mountain System ............................ $3-3$

3.3.2 Flow in Heterogeneous Partially Saturated Systerns ................................ 3-4

3.3.3 Nature of Evaporative Regine ....................................................... $3-5$

3.3.4 Influence of Thermal Regime on Evaporation and Infiltration ................... 3-6

3.3.5 Interaction of Waste Package with Migrating Fluids ................................ 3.7

3.3.6 Identification of Processes of Mobilization and Retardation ..................... 3-7

3.3.7 Evaluation of Relative Importance of Transport Processes ....................... $3-8$

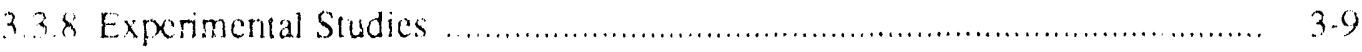


3.3.9 Verification of Numerical Codes

3.3.10 Validation of Mathematical Models

3.3.11 Model Development and Extension 3-13

3.3.12 Predictive Calculations 3-14

3.3.13 On-Line Validation of Models and Monitoring 3-15

3.4 Sequencing of Tasks 3-16

4.0 A SUMMARY OF FINDINGS: 1983-1989

4.1 1983: Conceptual Model for Moisture Movement in Fractured Porous Tuff

4.1.1 Capillary Mechanism: Preferential Saturation of Fine Pores .................... 4-1

4.1.2 Natural Fracture Model: Importance of Wall Roughness ............................ 4-3

4.1.3 Aperture Distribution: Approach to Quantify a Rough Fracture ................ 4-4

4.1.4 Flow Across Fractures: Importance of Effective Fracture Area .................. 4.. 4.7

4.1.5 Transpon Properties of Fractured Tuff: Derivation of Functional Relations

4.2 1984: Drainage Responses of a Fractured Tuff Column: Dynamic Interaction between Discrete Fractures and Porous Matrix

4.2.1 Fracture Description: Adaptation of Field Data

4.2.2 Discrete Fracture-Porous Matrix Model $4 \cdot 14$

4.2.3 Tool of Analysis: The TRUST Code 4-16

4.2.4 Major Findings: Flow Dominance by Matrix 4-17

4.2.5 A Useful Corollary: The Composite Model 4.21

4.3 1985: Vertical Infiltration through Welded-Nonwelded Units 4-22

4.3.1 Fracture and Matrix Data and Model: Observational Basis

4.3.2 Estimate of Ambient Infiltration Rate: Observational Constraints $4-23$

4.3.3 Welded-Nonwelded Flow Interaction

4.3.4 Approximate Model for Steady-State Profiles: Elementary Path Integration

4.3.5 Response to Extreme Infiltration Pulses

4.3.6 Estimate of Lateral Flows

4.3.7 Ambient Infiltration: An Estimate

4.4 1986: Distribution of Fluid Near a Fault Zone: Possibility of Lateral Flow

4.4.1 Unsaturated Fault

4.4.2 How to Model the Fault: The Seepage Face Idealization

4.4.3 Stratigraphy, Fracture, Matrix Data: An Update

4.4.4 The Ghost Dance Fault: A Column Profile

4.4.5 Multi-Column Models

4.4.6 Fluid Redistribution within the Tuff Formation

4.4.7 Vertical and Lateral Flow 
4.4.9 Ambient Flow Field ........................................................................ 4-41

4.5 1987: Statistical Analyses of Tuff and Soil Data ................................................ 4-41

4.5.1 Parameter Distributions ....................................................................... 4-42

4.5.2 Parameter Correlations ......................................................................... 4-44

4.5.3 Fracture and Fault Parameters ................................................................ $4-48$

4.5.4 Fractal Theory: Spatial Correlations and Scaling

Rough walled Fractures ..................................................................... $4-50$

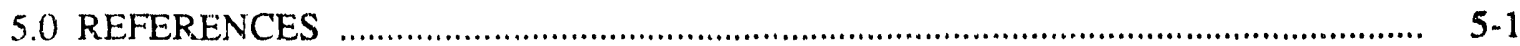

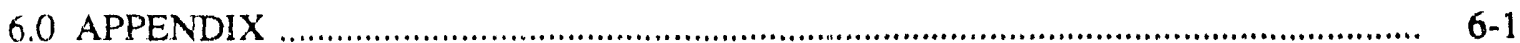




\section{List of Figures}

Figure 2-1 Flowdown of key hydrologic and transport issues.

Page

Figure 2-2 Framework of fundamental hydrologic and transport questions.

Figure 2-3 Hierarchical relations of 1echnical issues.

Figure 4-1 Evolution of the hydrologic performance assessment task.

Figure 4-2 Schematic representation of water flow in a fractured porous medium.

Figure 4-3 Schematic plan view of a fracture plane showing asperities (solid circles), liquid water (gray) and air (blank).

Figure 4-4 Aperture distributions for the Topopah Spring welded unit.

Figure 4-5 Effective flow versus pressure head for flow across the fracture plane.

4-10

Figure 4-6 Saturation versus pressure head for a rough-walled fracture.

Figure 4-7 Effective permeability versus pressure head for a rough-walled fracture.

Figure 4-8 Schematic representation of three fracture sets for the Topopah Spring welded unit.

Figure 4-9 Unsaturated drainage in a tuff column: comparison of results for 3 different conceptualizations.

Figure 4-10 Unsaturated drainage in a tuff column: pattems of darcy velocities (sign convention: horizontal fracture - flow into fracture is positive; vertical fracture - flow upward is positive).

Figure 4-11 Nonuniform grid used for one-dimensional simulations.

Figure 4-12 Three dimensional mesh used for studying column response to extreme infiltration pulses.

Figure 4-13 Horizontal saturation profile within matrix block of Topopah Spring unit. 
Figure 4-14 Response to 5,000-yr infiltration pulse: damping of fluxes within the Paintbrush unit.

Figure 4-15 Idealized cross section of Yucca Mountain.

Figure 4-16 Comparison of parameters of the van Genuchten model and the Brooks-Corey model.

Figure 4-17 Distribution of air-entry scaling factor for natural soils and tuff matrix.

Figure 4-18 Distribution of saturated hydraulic conductivity for natural soils and tuff matrix.

Figure 4-19 Correlation between saturated hydraulic conductivity and airentry scaling factor for natural soils and tuff matrix.

Figure 4-20 Correlation between saturated hydraulic conductivity and airentry scaling factor for tuff fracture and fault models.

Figure 4-21 Stcps inivolved in generating a rough fracture with fractal dimensions.

Figure 4-22 Relation between variograms and fractal dimensions. 


\section{List of Abbreviations}

\begin{tabular}{ll} 
Abbreviation & \multicolumn{1}{c}{ Expansion } \\
DOE & United States Department of Energy \\
GWTT & Ground water Travel Time \\
HLW & High Level Nuclear Wastes \\
LBL & Lawrence Berkeley Laboratory \\
NNWSI & Nevada Nuclear Waste Storage Investigation \\
NRC & United States Nuclear Regulatory Commission \\
NWRT & Nuclear Waste Repository Technology \\
PA & Performance Assessment \\
QA & Quality Assurance \\
SCP & Site Characterization Plan \\
SNL & Sandia National Laboratories \\
TRU & Transuranic Wastes \\
USGS & United States Geological Survey \\
YMP & Yucca Mountain Site Characterization Project \\
&
\end{tabular}




\subsection{INTRODUCTION}

In December 1987, the United States Congress chose Yucca Mountain, Nevada as the site for characterization as part of the program to provide the first U.S. high-level nuclear waste repository. As a consequence, the site characterization, repository design, and performance assessment (PA) activities for this site are expected to be intensified over the next few years to support the licensing application from the Department of Energy (DOE) to the Nuclear Regulatory Commission (NRC). Repository evaluation and environmental assessment of the Yucca Mountain site require comprehensive scientific investigations to quantify the uncertainties inherent in the natural system between the repository and the accessible environment. A good site, with a low fluid flow velocity field, will most likely be a difficult site to characterize and to evaluate. The challenge for the earth scientists is to focus on the key issues, quantify the uncertainties, and provide credible answers which help the public and the Government to decide if geologic disposal at Yucca Mountain is a viable and acceptable solution to this critical national problem.

\subsection{LBL Participation}

The Earth Sciences Division of Lawrence Berkeley Laboratory (LBL) has been involved over the past decade with various national and intemational research and technical assistance activitics in nuclear waste management, ranging from the Stripa fractured granite thermomechanical experiments in the late Seventies to the Yucca Mountain tuff hydrological studies in the mid Eighties. The tuff hydrological studies by the authors were initiated in 1983 by the Sandia National Laboratories (SNL) under the auspices of the Yucca Mountain Site Characterization Project (YMP), formerly the Vevada Nuclear Waste Storage Investigations (NNWSI) Project, administered by the DOE. 'This eifort is now part of the Nuclear Waste Repository Technology (NWRT) effort. SNL is one of the prime contractors for the YMP with responsibilities including PA activities.

In early 1983, N. Hayden, S. Sinnock and L. Ty'er of SNL contacted T. N. Narasimhan at LBL for subcontract support on conceptual modeling for the the PA activity of the partially saturated fractured tuff formation at Yucca Mountain. Accordingly, the LBL participation commenced with the small team comprising T. N. Narasimhan ard J. S. Y. Wang. S. Sinnock has been a driving force behind this project since then and has provided extremely valuable insights on technical concepts and directions in regard to programmatic priority. The project management 
task was assumed by Y. T. Lin in 1985 and later by P. G. Kaplan in 198\%. Both of them have provided invaluable technical and administrative support in the conduct of this project.

In parallel with the efforts of Narasimhan and Wang, an added subcontract cffort was commenced by K. Pruess and J. S. Y. Wang to address conceptual issues related to the nonisothermal fluid flow regime in the vicinity of the waste package, the so-called near-field transport problem. They were later joined by $Y$. W. Tsang. This subcontract was monitored first by K. Johnstone and later by $B$. Langkopf and by $A$. Peterson of SNL.

For over a decade now. many earth scientists at LBL have been actively involved in various national and intemational research relating to the disposal of high-level radioactive wastes (HLW) in geologic formations. G. S. Bodvarsson has carried out regional simulations and developed numerical capabilities to support the U. S. Geological Survey (USGS) site characterization activities. K. Karasaki and J. Long have developed random fracture network gerk. ation schemes for USGS and for international cooperative programs. E. Majer and T. V. McEvilly have used vertical seismic profiling techniques to detect fracture zones in rock masses. N. G. W. Cook and L. Myer have studied single fracture properties with acoustic waves and have made flow measurements through fractures in the laboratory. I. A. Apps, C. Camahan and H. A. Wol. lenberg have carried out field, laboratory, and numerical studies of geochemistry. C. F. Tsang and F. A. Witherspoon have assisted DOE Headquarters in progran review and evaluation. Interactions with L.BL. colleagues. SAL, scientists, and many other researchers have helped the authors of this repon to gain valuable knowledge and experience in unsaturated repository studies.

\subsection{Purpose}

The YMP is now in an important stage of transilion because Y'ucca Mountian has changed from being but one of the three sites to being the chosen site for characterization to determine its suitability for potential disposal of HLW. In this period of transition it is appropriate that we pasue, evaluate our current knowledge about the Y'ueca Mountain system, and use this evaluation as a guide 10 plan our activities for the futun:. This desire 10 draw upon our experiente gathered so far in order to better achieve our goals is the motivation for this repon.

The thrust of the report is 10 identify the key hydrologic and uansport issues that are imporiant to the projec and the tasks that are necessary for resolving these issues. It is in the nature of the ultimate decision-making process that complex issues be digested imto a sel of simple 
measures and answers amenable to the decision-making ventu' .. Recognizing this, we present the key hydrologic and transport issues in a hierarchical fashion; we start with the simple elements of the unsaturated repository concept, go through comprehensive and complex issues, and end with credible and quantifiable measures to enable decision-making en the suitability of the site for geologic disposal of HLW. Broad conceptual, experimental, and computational approaches to support PA of hydrology and chemical tsansport at Yucca Mountain are discussed. In the final part, we return to our project on tuff hyúrology studies. We review the major findings, describe the motivation and rationale that have dictated the evolution of o't contributions to the Yucca Mountain studies, and discuss the key issues which remain to be restived from our perspective. We hope that a proper mix of general discussion of the key issues and specific suggestion of critical investigations can help us to effectively contribute to the YMP within the scope and context of SNL's PA activities.

The key issues presented in this report have been constructed for the purpose of guiding stu. dies of hydrology and chemical transport. They do not replace or modify the "key issues" that are the organizing principle for the Yucca Mountain Site Characterization Plan (DOE, 1989) and for much of the technical work done by the entire Yucca Mountain Site Characterization Project. 


\subsection{KEY ISSUES: HYDROLOGY OF THE UNSATURATED ZONE}

\subsection{Repository Requirements}

To evaluate the unsaturated rapository' concept, we need to characterize the hydrologic regime at Yucca Mountain, examine the interaction of fluid with waste packages, and evaluate the long-term migration of the radionuclides from the repository to the accessible environment. Many simple questions and technical issues will arise in the course of investigations, and demand comprehensive resolutions and simple answers. A balance between practical constraints and scientific considerations is crucial for the success of this project in solving a very challenging earth science problem.

\subsubsection{Background of Concept}

In order to view the key issues in proper perspective, it is useful to know the manner in which the concept of radioactive waste disposal at Yucca Mourtain developed. An excellent account of this development is given by Roseboom (1983). That the thick unsaturated zone in the arid southwestem United States could be potentially suitable for the disposal of high-level radioactive waste was originally suggested by Winograd $(1972,1974,1976,1981)$ of the USGS. Yet, the Yucca Mountain site was initially considered as a potential site for radioactive waste disposal on the basis of host rock conditions below the water table. Later, as knowledge of the rnountain grew, it was recognized that not only will the unsaturated zone itself provide an excellent host for the wastes but that the repository will derive the benefits of both the unsaturated zone and the saturated zone. Accordingly, several scientists of the USGS jointly suggested in February 1982 to the DOE that the unsaturated zone at Yucca Mountain should potentially be a desirable site for HLW disposal. In addition, the USGS also recommended to the NRC that the proposed rules governing repositories, which had hitherto addressed only the saturated zone, be modified io include the unsaturated zone. Among the scientists at the USGS who recognized the potential value of the thick unsaturated zone for radioactive waste disposal, 1. J. Winograd particularly devoted a great deal of technical attention to the problem since the early 1970's. His early ideas focused on the disposal of defense-generated transuranic waste (TRU) in the alluvial fill of the Sedan Crater located several tens of miles northeast of Yucca Mountain. Subsequently, the favorable aspects of this idea were recognized to be also applicable to the burial of HLW within the volcanic tuff at Yucca Mcuntain, well above the water table. 
By 1982, the Yucca Mountain concept gradually passed the first phase of a broad preliminary assessment to the next phase of testing some of the preliminary ideas with more detailed analysis. This was also the time when the authors of this report got actively involved with the erstwhile NNWSI. The thoughts regarding the Yucca Mountain, as they emerged from the first phase of analysis, are summarized in Roseboom (1983), who also had the benefit of discussions with the scientists of the USGS who were devoled to the analysis.

Broadly, it was recognized that groundwater is the most important agent for the transport of radionuclides. Contaritination by gaseous species in the vadose zone was considered to be of lesser concern. Also, based on the suggestions of Winograd (1981), it was felt that solid wastes, suitably emplaced above the water table, could be expected to remain indefinitely dry in arid regions.

As evidenced from Roseboom's publication, the following are the highlights of the early ideas on Yucca Mountain.

\subsubsection{Factors Favoring Yucca Mountain}

- Very low water flux: Because of the very low precipitation and very large evapotranspiration, natural recharge to the deep groundwater table is extremely small.

- Rapid drainage: If the host rock in the unsaturated zone is well fractured, water will rapidly drain downward, resulting in very small residence times within the fractures.

- Strong capillary retention of water: Within the porous matrix with high bulk capillarity, the vertical movement of water becomes so slow that it is questionable if movement occurs at all.

- Minimal contact with waste package: Only a fraction of the water migrating downward through the repository would actually contact the waste. This potential contact could be further reduced by suitable engineering design of the waste-package emplacement.

- Geochemical retardation: An added geocheinical barrier to radionuclide migration would be provided by the Calico Hills unit which intervenes between the repository and the water table. This unit, fine-grained and rich in zeolites, possesses significant sorptive properies.

- Preservation of site integrity: Because highly permeable vertical fractures may help quickly drain infrequent shor pulses of flux, the presence of highly permeable channels per se within the unsaturated tone (abandoned boreholes) may not be undesirable. Therefore site integrity may not be jeopardized by drilling a large number of 
exploratory holes or shafts. So also, faults and fracture zones may not pose as much of a hazard as in the saturated zone.

- Accessibility to monitoring and recovery: The accessibility of the repository above the water table has two principal advantages. First, it facilitates systematic monitoring of site performance during construction, operation and postconstruction phases. Second, should monitoring reveal potential signs of site failure, the wastes could be recovered for altemate disposal or remediation.

\subsubsection{Caveats}

- Perched water bodies: Possible existence of large perched water bodies within the vadose zone may provide undesirable water access to the repository. However, if shafts or boreholes intersect these, they would drain quickly, as experience would suggest. At worst, these could also be artificially drained through carefully designed boreholes.

- Rise of water table: Possible rise of the water table by a few hundred meters during the next 1,000 to 10,000 yr may lead to the flooding of the repository.

- Wet Climate: Drastic change to wet climate (pluvial environment) within the next 1,000 to 10,000 yr may lead to increased infiltration.

- Gas Transport: Relcase of gaseous contaminants would necessitate consideration of gas and vapor as transporting agents of radionuclides in addition to percolating groundwater.

\subsubsection{Nature of Technical Challenge}

Our current knowledge based on aspects of climate, hydrogeology, geochemistry and soil physics suggests that the disposal of high-level radioactive waste in the thick unsaturated zone of Yucca Mountain is potentially a most advantageous proposition in the national context. Nevertheless, the task of quantifying the advantages to enable the licensing of the repository is not an easy one. It is an extraordinary ciailenge for two primary reasons. First, unlike other branches of engineering such as manufacturing or construction, in which materials and components can be fabricated to specification, we are concerned with a natural geological system which is intrinsically heterogeneous, and the heterogeneities are very difficult to characterize. These heterogeneities introduce considerable uncertainty into our ability to quantify it. Second, the time scale of reguired prediction, assumed to be 10,000 yr, (DOE, 1989) is far beyond the gamut of traditional engineering practice. 
The intrinsic complexity and the associated uncertainty render the technical problem of evaluating the Yucca Mountain site a remarkably challenging one. A third dimension of the challenge is the sociopolitical sensitivity of the issue of toxic waste disposal.

These challenges have been candidly addressed by Davis (1982) as well as by Winograd (1986). Davis (1982) points out that uncertainties inherent in a natural system do not necessarily negate our ability to find a viable engineering solution to a critical national problem. Nor should conservatism, which is essential for building a safety factor into engineering design, be taken to such an extreme that rational solutions have little chance of success. Winograd (1986) suggests how the issue of radioactive waste disposal is a transscience problem. It is a problem for whose solution we neither have an adequate data base nor could we hope to have one in the foreseeable future. We therefore have to buttress our conventional engineering methodologies by other semiqualitative knowledge, in particular, archaeology.

The task of Yucca Mountain performance assessment therefore demands a con ination of conventional engineering as well as an ability to temper that with shrewd judgment. This in itself is a challenging technical task. However, there exists a larger, but less tangible challenge. This concerns the credibility of science and its ability to solve a technical problem from a practical point of view. Such credibility will be critical at the time of licensing, when decision-making will involve technical experts from branches of science not related to the earth, as well as the informed laypublic.

Because of the complexity of the problem as detailed above, the performance assessment has to draw upon both the existing technologies and new research to fill gaps in the existing state of knowledge. This has to be achieved with well-defined constraints on resources and time. The schedule for the repository (DOE, 1989) requires that the licensing application be si.brnitted to the NRC within decades. Additionally, licensing is intimately linked to legal rerjuirements of comprehensive demonstration that the site is acceptable. A consequence is that faithful recordkeeping, documentation and procedural compliance are integral part of the performance assessment venture. This inevitable quality assurance (QA) aspect adds additional constraints to the available physical resources and ume.

The importance of $\mathrm{QA}$ in a complex venture such as licensing cannot be overemphasized. However, the nature of the QA procedures appropriate for the YMP needs careful assessment. The currently in-place QA procedures for the YMP are modeled after similar procedures developed from long experience of construction of power plants, submarines and other systems. 
Not only are these systems constructed mostly out of man-made materials, but many such systems are periodically built. Thus, the QA procedures are continually updated. In comparison, YMP involves a unique system with built-in uncertainties, both because of the nature of the materials and because of the long time scale of prediction. The YMP requires QA procedures that are specially tailored to suit its technical uniqueness as well as the constraints of time and resources available.

\subsubsection{A Rationale for Solution}

Within the constraints of these challenges, a prudent solution approach to the overall problem may contain the following elements:

- A serial arrangement of barriers which will, in combination, inhibit the potential migration of contaminants,

- Continuous and systematic monitoring of the site during and after construction and operation to detect any unforeseen failures, and

- Retain an ability to retrieve the wastes for altemate disposal, should unforeseen failures be detected.

The concept of the serial arrangement of features is inherent in the multiple barrier strategy that is outlined in the Site Characterization Plan (SCP) (DOE, 1989). The concept of systematic and continuous monitoring during the construction and operation phase is extremely important and is quite compatible with the philosophy of the SCP, although one may debate the feasibility of monitoring by future generations after repository closure.

In designing any complex system one must ideally provide for open ends to abort the system safely should unforeseen complexities develop. In the case of Yucca Mountain, one such open end is the accessibility of the waste above the water table; retrieval or remedial actions would be feasible in case of failure.

The YMP is concerned with a one-of-a-kind problem of unusual complexity. Many uncertainties exist related to the characterization of a natural heterogeneous system as well as to the boundary conditions and source terms that will dictate the future of the system. Yet, these uncertainties may not necessarily preclude our ability to evaluate the suitability of the site. What is perhaps needed is an extension of decision-making methodologies which will help combine quanlitative engineering knowledge and other semi-quantitative knowledge in a creative fashion. 


\subsubsection{The Accessible Environment}

The ultimate concern of the YMP is the potential contamination of the human environment by radioactive contaminants, should toxic radioactive constituents find their way to the biosphere. For purposes of performance assessment in examining compliance with regulations, the biosphere is defined in terms of the accessible environment for which the regulations provide an official definition.

In this discussion of Yucca Mountain, it is useful to recognize two parts of the accessible environments. The first is the local land surface; this includes not only the sloping upper surface and the slupes all around the mountain, but also the land surface in the general vicinity of Yucca Mountain to an as yet unspecified distance. The second part of the accessible environment includes all those locations at which the the groundwater may potentially come into contact with the human habitat. The groundwater beneath the water table at Yucca Mountain is known to move gencrally towards the south and the southwest, discharging at playas and other depressions away from the mountain. Obviously, the points of discharge of these groundwaters constitute the accessible environment. Also, along the pathway from the Y'ucca Mountain to these points of discharge, the aquifers may in the future be tapped by drilled wells for human use. Therefore, the second part of the accessible environment not only includes the distant points of groundwater discharge, but also the areas between the mountain and the points of discharge where potential for development of groundwater for water supplies exists.

The first part of the accessible environment, namely, the local land surface, would be contaminated, if at all, by gascous elements such as ${ }^{14} \mathrm{C},{ }^{129} \mathrm{I},{ }^{85} \mathrm{Kr}$ (Roseboom, 1983). These elements will be transported within the air-phase of the vadose zone by water vapor or simply as gascous constituents. So far, the evolution of ideas in the YMP has been such that the potential contamination of groundwater has received greater emphasis than the potential contamination of the locial atmosphere.

\subsubsection{A Measure of Performance}

The overall task of demonstrating the suitability of the Yucca Mountain site is an extremely complex one. Yet, from the point of view of regulation and licensing it is imperative to incorporatc the effects of these complexities into a small number of critical guiding functions. Some guiding functions are quantitative measures of hydrologic performance. One such guiding funclion discussed widely among the participants in the YMP is the groundwater travel time (GWTT), 
for which NRC regulations prescribe a numerical performance objective. Other guiding functions, such as dose measure and canister lifetime, are also imponant in the assessment of repository performance; some of these other guiding functions are also used as performance measures in regulations that govem repositories.

\subsection{Fundamental Questions}

Before we proceed to identify the key issues pertaining to unsaturated zone hydrology, it is instructive to state the fundamental questions that dictate the achievement of the ultimate goals of the project. The following four questions serve this purpose.

- What is the nature of the fluid movement within the Yucca Mountain?

- What is the potential for fluid-waste contact and mobilization of toxic constituents?

- What is the potential for the accessible environment to be unacceptably contaminated over a 10,000 yr-period?

- How can the potential hazard be quantitatively represented so as to enable decisionmaking on site suitability?

The fact that Yucca Mountain has been declared a site for characterization is clearly an indication of the existence of strong preliminary evidence of the suitability of the Yucca Mountain site. One of the critical tasks of PA is therefore to comprehensively test the preliminary evidence and quantify the results in a credible manner.

We are concerned with a multiple-barrier system in which the barriers are placed in series. In this context the fundamental questions stated above are related to each other in Figure 2-1.

Against the backdrop of these fundamental questions we may now proceed to consider the multitude of technical issues that need to be addressed in order to answer the aforesaid questions. For purposes of clarity, we first list the various issues in the next section, to be followed, in the succeeding section, by a discus.ion of the listed issues.

\subsection{Technical Issues}

As described in Section 2.1.6, the accessible environment could be contaminated either by the upward migration of gaseous phases or the downward migration of liquid water through the unsalurated rone. Although the term hydrology is usually applicable to liquid water, in the present work we will use that $1 \mathrm{erm}$ in a broader sense, including transport by the gaseous phase. 
Quantity of Fluids Capable of Transporting Contaminants<smiles>[CH][CH]</smiles>

Potential for Contact with Waste Form

$\downarrow$

Potential for Transport beyond the Unsaturated Zone

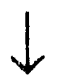

Magnitude of Impairment of the Accessible Environment

Figure 2-1 Flowdown of key hydrologic and transport issues. 
The technical issues of relevance can be broadly grouped into four categories, corresponding to the four fundamental questions already discussed. This grouping is schematically shown in the branch diagram in Figure 2-2. The issues themselves are listed in the next section.

\subsubsection{A List of Issues}

The hierarchical relationships between the technical issues are illustrated in Figure 2-3 and the issues themselves are enumerated below.

\section{Nature of Hydrologic Regime}

\section{I.1 Before Repository Construction}

\subsubsection{Groundwater as the Transporting Agent}

- What are the direction and magnitude of water motion within the unsaturated zone at Yucca Mountain?

Is it directed downward, upward, or is there any movement at all?

Is there a depth from the land surface which separates the evaporative regime from a deeper, infiltration regime?

If a long-term infiltration (or exfiltration) exists, what is the magnitude of the flux?

Is the flux direction dominantly vertical or do significant lateral fluxes exist?

What are the implications of flow patterns to contaminant migration?

- How are the fluxes distributed within the mountain?

Are they uniform!ly distributed?

Do strongly preferred pathways exist for the movement of water?

If so, how are these preferred pathways distributed? In the fractures? In the matrix?

Are these pathways relatively permanent? Or, are they short-lived, temporary features governed by infrequent, extreme precipitation events?

What roles do faults and shear zones play as preferred pathways for fluid flow?

- What is the state of the present flow regime within Yucca Mountain?

Is the current infiltration regime a steady-state condition or is it a transient condition on the time scale that is of interest?

How did the deep unsaturated system evolve over the recent geological past? 


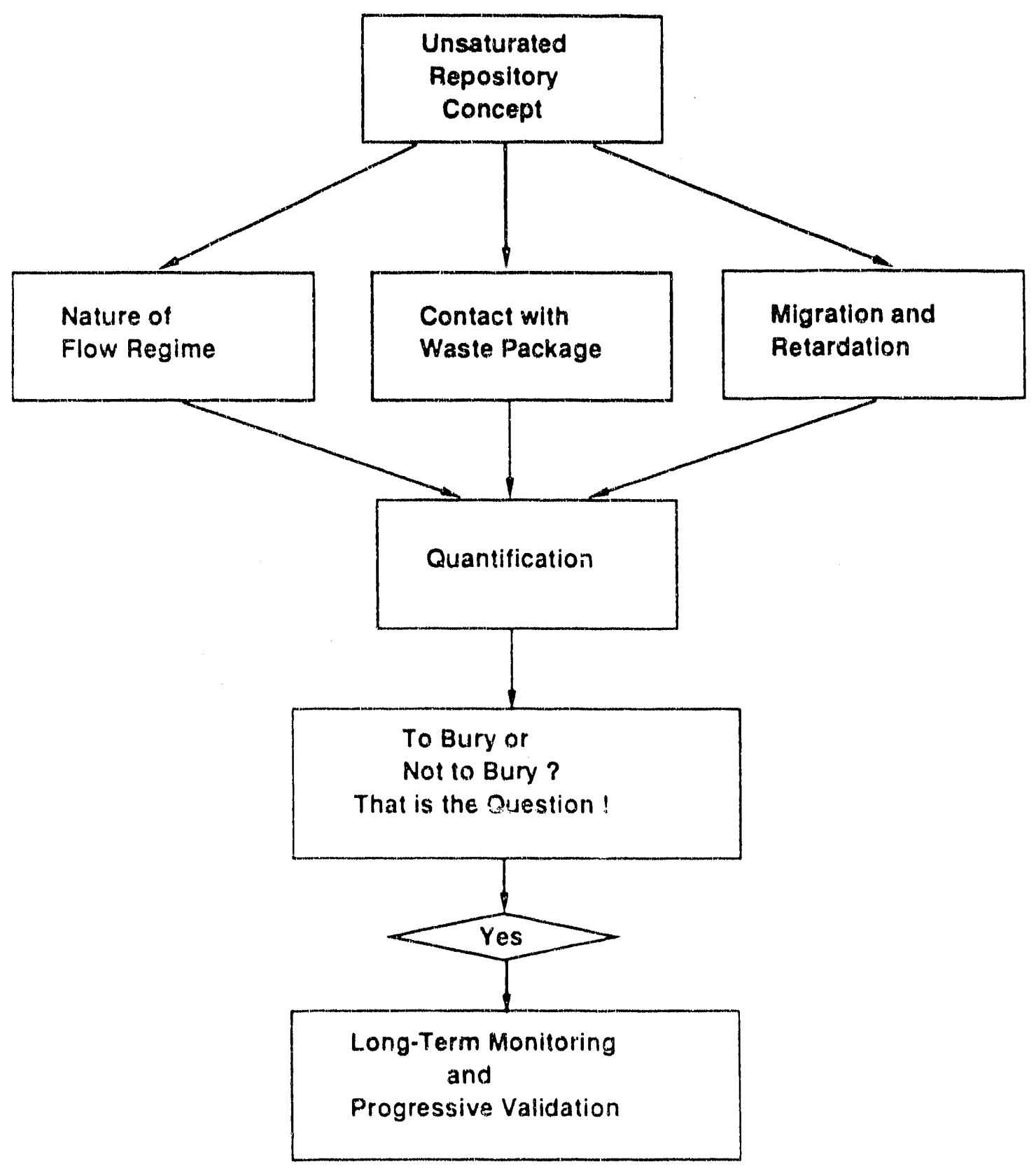

Figure 2.2 Framework of fundamental hydrologic and transport questions. 


\section{Hierarchy of $\mathbf{H y d r o l o g i c / T r a n s p o r t ~ I s s u e s ~}$}

\section{Nature of Hydrologic Regime}

\section{I.1 Before Repository Construction}

1.1.1 Groundwater as the Transporting Agent

I.1.2 Vapor/Gas as the Transporting Agent

\subsection{After Repository Construction}

I.2.1 Groundwater as the Transporting Agent

I.2.2 Vapor/Gas as the Transporting Agent

II. Interaction of Fluids and Waste Package

II.1 Groundwater and Waste Package

I1.2 Vapor/Gas and Waste Package

\section{Migration of Contaminants}

III.1 Groundwater as the Transporting Agent

1II.2 Vapor/Gas as the Transporting Agent

IV. Quantification

IV.1 Measures of Performance

IV.2 Mathematical Models; Quantification and Uncertainty

IV.3 Long Term Validation; Monitoring the System

Figure 2.3 Hierarchical relations of technical issues. 
Has the water table been rising or falling?

- How does the system respond to extreme precipitation events?

Do perched water bodies develop in response to extreme precipitation events?

Do extreme precipitation events give rise to lateral diversion of groundwater?

Are fingering phenomena and associated instability important in response to extreme precipitation events? If so, will they initiate the formation of preferred pathways?

\subsubsection{Vapor/Air as the Transporting Agent}

- What is the nature of the present evaporative regime within the Yucca Mountain?

Is there a depth below which the temperature distribution is not sensitive to near-surface fluctuations?

What is the magnitude of the evaporative flux on a long-term basis?

To what depth does air circulate within the mountain in response to diurnal changes, seasonal changes and other nonperiodic changes in atmospheric pressure?

What is the role of the circulating air in the transport of moisture to the land surface?

On a geological time scale, has the evaporative regime played any role in the evolution of the water table? If so, what are the implications in regard to the present day moisture fluxes through the unsaturated zone?

Does the evaporative regime extend all the way to the vater table? Or does it extend to the water table only through a few well-connected fractured channels but not through the overall rock matrix?

How do the evaporative regime (involving vertically upward vapor flow) and the infiltration regime (involving vertically downward liquid flow) interact?

How does the air/vapor transport regime respond to infiltration puises following extreme precipitation events? What is the implication of this response to the diversion of liquid flux towards highly permeable, large openings?

\subsection{After Repository Construction}

\subsubsection{Groundwater as the Transporting Agent}

- How will the currently existing infiltration conditions be disturbed by the construction and the operation of the site?

What will be the effect of maintaining an essentially atmospheric pressure condition at the repository on the infiltration process? 
What hydrologic criteria are needed to define the disturbed zone?

Will the thermal regime and the associated ventilation structures cause a progressive desaturation of the disturbed zone? What will be size and the geometry of the disturbed zone? What will be the extent of desaturation?

Will the process of desaturation cause the capture of the ambient downward flux of infiltrating water and establish a pronounced vertically upward flux of liquid water away from the water table?

How long will this effec: persist after repository closure?

Following the dissipation of the thermal regime, will the current fluid flow regime reestablish itself? How long will the resaturation process last?

Will buoyancy forces associated with temperature induced density changes cause the upward movement of liquid water from the repository to the land surface?

What will be the nature of the postresaturation infiltration regime?

What will be the hydrological boundary conditions at the edge of the disturbed zone?

\subsubsection{Vapor/Air as the Transporting Agent}

- How will the thermal regime of the waste package modif; the existing evaporative regime?

Will the strong thermal regime overpower the existing infiltration regime and establish a long-term upward flux of air and vapor from the disturbed zone?

During the active phase of the thermal regime, how will the vapor regime respond to extreme precipitation everts?

Will the relative permeability characteristics of the fractures permit the rapid expulsion of the vapor phase through fractures?

Or, will porkets of gas/vapor locked in isolated larger openings significantly inhibit formation of preferred pathways?

\section{Interaction of Fluids and Waste Package}

\section{1 Groundwater and Waste Package}

- How will the infiltrating vadose water come into contact with the waste package?

What are the roles of waste-package geometry, waste-package placement, engineered barricrs and diversion suructures in governing the contact of liquid water with the waste? 
What ..11 be the duration of the contact?

What is the probability that preferred pathways, should they exist, will contact the waste package?

Is rapid drainage of water through preferred pathways following extreme precipitation events conducive to increasing contact of water with waste packags?

In order to jeopardize the effectiveness of the repository, how much of the infiltrating water should be in contact with the waste package? For how long?

Could the probability of contact be significantly reduced by engineering simple structures to divert the flux away from the waste package?

Is there a potential for very slow but very long term contact of the waste package with the capillary-bound matrix water? Will this pose a threat to the repository on a long-term basis?

II.2 Vapor/Gas and the Waste Package

- What will be the nature of the interaction between the vapor/gas phase and the waste package?

Is the pe formance of the waste package sensitive to the presence of water vapor in a two-phase environment or in a dry-up environment?

Will the purge oxygen from the vicinity of the waste package cause the corrosion rate of the waste package to change?

\section{Migration of Contaminants}

III.1 Groundwater as the Transporting Agent

- What are the important mechanisms by which contaminants may be transported by liquid water within the Yucca Mountain?

What is the relative importance of the following transpor processes?
Adrection?
Molecular diffusion?
Hydrodynamic dispersion?

How important is the pxiential transport of contaminants by advection in preferted pathways?

What are the potential mechanisms by which the contaminants may be mobil. ired into the liquid phase? Is the chemistry of the vadose water within Yucca Mountain conducive to such mobilization? 
What are the potential retardation mechanisms by which the migration of contaminants in the liquid phase may be inhibited?

Adsorption onto the solid surface?

Precipitation?

Diffusion into dead-end pores?

Diffusion into the solid phase?

How conducive are the physicochemical conditions within Yucca Mountain to promote such retardation?

What do the present day mineralogy and geochemistry of the Yucca Mountain rocks reveal about the aqueous chemical transport processes that were active in the past?

Can an integrated analysis of fluid flow and geochemistry quantitatively help resolve the question relating to the magnitude of infiluration flux within the Yucca Mountain?

III.2 Vapor/Gas as the Transporting Agent

- What are the important mechanisms by which contaminants may be transported by vapor and gas?

What is the relative importance of the following transport processes:
Advection?
Molecular diffusion?
Binary diffusion?
Knudson diffusion?

Will the volatile contaminants released by the waste package react with water vapor, other gases and the solid constituents?

How important are the variations in the concentrations of vapor and oxygen in the transport of the gaseous contaminants?

Is aerosol transport an important mechanism for the transpurt of nonvolatile constituents?

IV. Quantification

The technical issues discussed above, belonging to categories I, II and III, are all processoriented. The issues related to quantification which follow are of a different kind, common to all the processes. Issues of quantification are common to all three categories above.

IV.1 Measure of Performance

- From the viewpoint of licensing application, what are the different measures that can be used to assess the hydrologicai performance of Yucca Mountain?" 
How effective is GWTT as a performance measure?

How may one define GWTT in the case of a highly heterogeneous, partially saturated system?

How may one define GWTT in a transient system?

How important is it to incorporate into GWTT such factors as the probability of the contact of fluids with the waste package, the effect of duration of contact of the fluids and the waste package, and the retarding influence of the natural barrier?

If so, how should GWTT' be defined?

- What other performance measures are relevant"?

\section{IV.2 Mathematical Models}

- What are our current abilities of modeling in regard to hydrological and chemical transport problems relevant to the YMP?

What are special features of the mathematical problems which represent the hydrological and geochemical processes of relevance to performance assessment?

What is the current status of our modeling capabilities?

Are the existing tools adequate?

How credible are these models? How internally consistent are they? How closely do they represent the field systems they seek to mimic?

What is the critical quantum of field data needed to reliably apply the models to the field problem?

-What are the critical model development needs?

IV. 3 Long-Term V'alidation; Monitoring the System

- What are the essential features of a monitoring system that will provide adequate hydrogeological and hydrogeochemical information during the constructionoperation phase to enable validation of predictions made during the characterization phase?

- What are the essential features of a long-term monitoring system to help detect potential failure." 


\subsubsection{Discussion}

Direction and Magnitude of Fluxes: A very important attribute of the Yucca Mountain that influenced its preliminary choice as a site for characterization is the extremely low infiltration of water through the unsaturated zone. A basic goal of the PA effort is therefore to adequately define the nature of the existing fluid flow regime within the Yucca Mountain, with reference to both the liquid phase and the gaseous phase. Despite careful laboratory and field investigations as part of the YMP, considerable uncertainty still exists about direction and magnitude of the flux of liquid water within the unsaturated zone. Currently used estimates only suggest an upper bound of about $0.2 \mathrm{~mm} / \mathrm{yr}$ for long-term downward infiltration toward the water table. Is it possible that because of the strong evaporative regime there is no long-term downward infiltration within the Yucca Mountain?

Preferred Pathways: A second important attribute of the Yucca Mountain that causes a great deal of concern in regard to contaminant transport is the presence of fractures in the welded tuif units. The concern is that these fractures may constitute preferred pathways for the rapid migration of contaminants from the repository to the accessible environment. On the other hand, an early argument that was advanced in favor of the Yucca Mountain (Roseboom, 1983) was that the highly permeable fractures may act like "storm sewers" and would help the rapid drainage of water following infrequent extreme precipitation events. More recent computer modeling studies by Wang and Narasimhan (1986) suggest that the very strong capillary regime in the extremely fine-grained tuff will tend to inhibit the saturation of fractures even after strong precipitation events. In what way then is the presence of fractures within the welded units detrimental to performance of the repository?

State of the Hydrologic System: Closely related to the isslie of direction and movement of infititrating water is that of the present state of the flow system within Yucca Mountain. If the currently estimated infiltration fluxes of less than about $0.2 \mathrm{~mm} / \mathrm{yr}$ are reasonable, then the system could be treated as a steady-state one on a time scale of conventional engineering practice. However, on a time frame of 10,000$)$ yr, does the system reveal notable transient aspects? Do the observed variabilities in saturation and moisture suction within the mountain indicate effects of infiltration pulses that occurred in response to extreme precipitation events many centuries ago? In what way will these transients influence contaminant migration? Can the transient regime provide clues about long-term infiltration rates? 
Response to Extreme Precipitation Events: It is normally assumed that gravity dominates the movement of water in the vadose zone and the infiltration fluxes are directed almost vertically down. However, is it possible that following extreme precipitation events saturated flow may be initiated at material interfaces leading to lateral diversion of flows? Or, is lateral diversion to occur under purely unsaturated conditions of flow? Note in this regard that the tuff units of Yucca Mountain dip gently toward the east. The concem is that such lateral diversions, should they occur, might provide access to permeable faults or shear zones and through them provide a fast pathway to the accessible environment.

Vapor/Gas Phase Movement: The presence of the deep vadose zone at the Yucca Mountain is due in part to the existence of an extremely strong evaporative regime. It is to be expected that this regime will transport substantial quantities of moisture vertically upward from the vadose zone over long periods of time. Developing an understanding of the nature of this evaporative regime is therefore a very important issue. Weeks (1987) made convincing field observations at the Yucca Mountain which show that air circulates down to several tens of meters within the Yucca Mountain because of seasonal as well as diumal changes in temperature and pressure. To what depths does the evaporative regime extend within the mountain? How much of moisture does it iransport? How does it interact with the downward-directed infiltration flux?

Postemplacement Hydrologic Regime: The proposed repository is a very large underground opening. From the perspective of the hydrologic flow regime, this structure will greatly change the geometry of the system with near-atmospheric boundary condition at the repository surfaces, partly because of the highly permeable pathways between the atmosphere and the repository and party due to the ventilation system to maintain reasonable working conditions for underground operations. Finally, the heat production from the wastes themselves will profoundly alter the thermal regime within the vadose zone. How will the ambient flow iegime be affected by the repository'? If the imposed thermal regime causes a pronounced desaturation of the region near the repository, then this region may act as a sink for moisture accumulation for a very long period of time, capturing groundwater that may otherwise move towards the water table. Also, the thermal field will induce buoyancy flow's and modify the air circulation patterns within the mountain and will strongly influence the direction and magnitudes of the fluxes.

Contact with Waste Form: The strategy for burial of HLW at the Yucca Mountain is based on the multiple barrier concept. One of these barriers is the waste package itself. The solid waste will be packaged in stainless steel cylinders about 10 inches in diameter and about 10 feet 
long. In order that contaminants be mobilized from these into the groundwater, sufficient quantities of groundwater must come into contact with the waste package for sufficiently long periods of time. Therefore, the nature of the potential contact between the infiltrating groundwater and the waste package is a very importan: issue. If oriented favorably in relation to the local groundwater flow pattem, the packages would present a low profile and the probability of contact would be minimized. The issue of groundwater-waste package contact is also quite important in evaluating the potential of transport of contaminants by pulses of water that may pass through the system following periods of extreme precipitation.

Analogous to the issue of groundwater-waste package contact is that of the potential contact of the vapor or gas phase and the waste package. During the active phase of thermal loading as well as during other periods, gases and water vapor would invariably be in contact with the waste package. Moreover, owing to active air circulation through the system, a large mass of air would continuously be moving through the system. But the proportion of this air that will actually contact the individual waste package is as yet uncertain. The implication of this vapor/gas phase contact with the waste package to the mobilization and transport of gaseous contaminants is an important issue that needs to be addressed.

Processes of Transport: Once mobilized into the fluid phase, contaminants will be transported by advective, diffusive and dispersive processes. In the specific context of Yucca Mountain, which of these transport processes are most important? Within the unsaturated zone, one would suspect that molecular diffusion may play a more important role than advection and hydrodynamic dispersion, both of which depend on groundwater velocity. However, advection and associated longitudinal dispersion could be the dominant mechanisms in preferred pathways such as fractures, faults or shear zones. In the vapor/gas phase, however, advection may be dominant because of the high flow velocities involved.

Fluid-Rock Interactions: One of the favorable aspects of Yucca Mountain is the presence of natural conditions under which very strong interactions between fluids and solids are possible. Beciuse of extremely low infiltration velocities and the enomous surface area of the solids $15(0),())()$ to more than 2 million square meters per cubic meter of rock, depending on method of an:ilysis; Claassen and White, 1979) fluid-solid interactions can occur over large surface areas for long periods of time. Such interactions will facilitate surface sorption on surface-active constitucn such as reolites as well as precipitation where redox conditions are suitable. 
Note that the volcanic tuff is actually made up of particles of glass. Where these glasses devitrify, the process of devitrification is usually accompanied by the generation of defects and pores on the surfaces of the glassy particles. Recent experimental studies by White and Yee (1986) suggest that diffusion into the solid phase of the tuff matrix could be a measurable mechanism. If this is so, diffusion into the solid phase could be a beneficial mechanism in regard to inhibiting the transport of contaminants by the liquid phase.

Yet another mechanism that may help retard contaminant migration within Yucca Mountain is diffusion into dead-end pores. Considering the strong capillary forces within the tuff rock, it is reasonable to expect that the water phase could be stagnant in many dead-end pores. Although they may not play any role in flow, these dead-end pores may help absorb contaminants from the mobile phase by molecular diffusion.

The caveat in regard to fluid-solid interaction is that groundwater in the vadose zone at Yucca Mountain is likely to be highly oxidizing. If so, it will have a relatively high corrosion potential, conducive to the mobilization of metallic ions.

Geochemistry and Patterns of Fluid Flow: As we have already secn, a basic PA issue concems the direction and magnitude of water flux within the vadose zone. Indirect estimates made from soil physics parameters measured in the field suggest extremely low downward fluxes. Because such low fluxes cannot be directly measured with currently available field techniques, it is very desirable to have these estimates corroborated by other independent means. Currently well-established geochemical techniques involving isotopic analysis of vein minerals, liquid inclusions and pore waters should provide valuable information on the evolution of the vadose zone during the recent geological past. An added advantage of gaining knowledge of such an evolution is that it would provide credible insights on predicting the response of the system over the next 10,000 yr. These insights will be valuable in minimizing uncertainties associated with conventional predictive modeling.

In the balance the potential for rock-fluid interactions and the associated inhibition of contaminant migration within the vadose zone at Yucca Mountain appear to be considerable. The evaluation of these genchemical processes and their influence on PA constitute an extremely important issue.

Performance Measure: At the present state of the site characterization effort, the most widely used measure of hydrologic performance is the premplacement GWTT. The concept of GWTT is generally associated with a steady-state field of fluid fow. Although ciWTT is an 
extremely useful notion in dealing with systems with simple geometry and heterogeneities, it is useful to examine its efficacy in regard to the extremely complex hydrologic system characterizing the vadose zone at Yucca Mountain. Several important questions arise. How amenable is the concept of GWTT for handling highly heterogeneous, unsaturated systems characterized by pronounced convergence and divergence of flow paths? How can the GWTT concept be extended to accommodate the transient flow regimes characteristic of the postconstruction period? Within the framework of the GWTT, how may one incorporate the effects relatively short-lived flows through preferred pathways following infrequent extreme precipitation events? Is it overly conservative to exclude retarding effects of multiple barriers from the definition of GWTT? Is there a need for defining alternate performance measures?

Mathematical Models: As pointed out in the SCP (DOE, 1989), fluid flow and chemical l.ansport calculations using mathematical models will play a very key role in the licensing process. In the PA venture, such mathematical models will be used in two broad ways: (1) conceptual and theoretical studies to develop insights about the relevant processes and issues; and (2) detailed specific calculations leading to inferences about the potential perforrance of the site.

The classical mathematical model is in essence a quantified description of the effects of a system in response to certain prescribed causes. Given a unique set of parameters to characterize the causes and the nature of the system, the classical mathematical model leads to a deterministic solution of the problem. However, for a variety of reasons, including weaknesses in the conceptual basis, inaccuracies in the computational code, and uncertainties associated with the parametric data base, a certain amount of uncertainty is invariably associated with the relevance of the deterministic solution for the field problem on hand. The mathematical models used in the present PA effort must be capable of providing a quantitative estimate of these uncertainties to enable a rational decision-making about site suitability.

As part of the erstwhile NNWSI, considerable effort has been expended in assessing the nature of the models required for the PA venture as well the adequacy of existing codes. Two important issues that are being addressed are those of code verification and model validation. All these efforts are based on mathematical tools that have already been developed in the literature. A critical issue is to assess the needs for additional model development, both in terms of new methods especially geared for handling the special features of the Yucca Mountain system and in terms of tailoring existing codes to greatly increase their efficiency for solving particular classes of problems. 
Long-Term Validation and Monitoring: The site characterization phase of the YMP will provide a certain amount of hydrological and geochemical data, upon which predictions will have to be made about the potential suitability of the site. These predictions will be constrained by known uncertainties. If these uncertainties are within reasonable bounds, and it is decided that the site is acceptable to receive the wastes, then the construction phase will begin.

It is important to recognize that the construction phase and the subsequent operational phase will span several decades. Utilized properly, this duration of several decades can provide additional data about site performance on a time scale that is much larger than the one that was available during the site characterization phase (several decades as opposed to several years). Therefore, a systematic monitoring of the system during the construction and operation phase of the repository should be considered an integral part of the overall PA venture. This monitoring aspect will render the vital open-endedness which is essential to the design of any large scale, complex system.

Ideally, by the time of repository closure, the accumulated data base and the validated models will guide the design of any monitoring system that may be needed over a long period following the closure. 


\subsection{IMPORTANT TASKS FOR THE RESOLUTION OF HYDROLOGIC AND TRANSPORT ISSUES}

\subsection{Introduction}

The tasks that need to be undertaken to resolve the technical issues discussed in the previous chapter are varied in nature and require the use of laboratory and field experimentation, geologic field observations, and mathematical modeling exercises. For purposes of discussion, it is convenient to group these tasks into four categories:

1. Conceptual and theoretical analysis;

2. Laboratory and field experimentation;

3. Verification, validation and development of mathematical models; and

4. Computations to support licensing application.

At the present stage in the YMP our knowledge of the basic processes that have the greatest influence on hydrolugic performance is still limited. It is therefore necessary that these basic processes be investigated early on in the growth of the project. These tasks involving basic processes require conceptual and theoretical analysis as well as experimental and observational components. Until recently, very little data were available on the transport properties of the tuff rock at Y'ucca Mountain especially relating to unsaturated conditions. Since 1983, a small and useful data base has been built up in this regard through the efforts instituted under the NNWSI project. These data bases must be expanded to a critical size necessary to provide credibility to the licensing application. The conceptual-theoretical studies and the experimental data base will provide the input information essential for the computational tools. A variety of computational models are currently available in the literature to handle the broad category of problems relevant to PA. However, there is evidence that some model extension and model development efforts are needed in order to meet certain computational aspects peculiar to Yucca Mountain. Additionally, the reguirements of QA necessitate the verification, validation and documentation of the particular computational tools chosen for generating results that will be used to support the licensing application.

These results to be used in the licensing application will include diverse physical situations relevant to contamination of the accessible environment as well as error bounds on the credibility of calculations. These crror bounds will have to be estimated through sensitivity analyses and 
probabilistic methods. The predicted results and the associated uncertainties would need to be reevaluated using the increased data that will be forthcoming during the construction-operation phase. Therefore, the detailed computational results supporting the license application will need to be complemented by a plan for integrated monitoring of the system.

\subsection{A List of Tasks}

(1) Conceptual and Theoretical Tasks

(a) Preemplacement Conditions

- Analysis of long-term evolution of Yucca Mountain, integrating geochemical field observations (mineralogy, isotopic evidence), groundwater flow patterns, paleoclimatology and mathematical modeling

- Flow patterns in a large heterogeneous, partially saturated system

- Nature of evaporative regime

(b) Postemplacement Conditions

- Nature of the disturbed zone consequent to construction and operation of the repository

- Interaction of waste package with liquid, vapor and gaseous phases; role of package geometry, emplacement patterns and engineered barriers/diversion

- Essential process of mobilization, transport and retardation

(2) Laboratory and Ficld Measurements

(a) Understanding the Processes

- Evaluate importance of film flow on fracture walls and surface diffusion

- Evaluate gas/vapor migration and relative permeability of partially saturated fractures

- Obtain visual evidence of capillary dominance in partially saturated fractured media using tomographic techniques

- Delineate response of strongly heterogeneous partially fractured systems to pronounced pulses of infiltration, role of fingering and instability on generating pieferred pathways 
- Evaluate essential geochemical processes of mobilization and retardation

(b) Data Pase on Material Properties and Initial Conditions

- Use of osmotic potentials to generate large suctions and measure unsaturated transport properties

(3) Mathematical Models

(a) Verification of Mathematical Models

- Verification of deterministic as well as probabilistic models for internal mathematical consistency

(b) Validation of Mathematical Models

- Validation of deterministic as well as probabilistic models for physical reality

(c) Model Extensions and Development

- Evaluate current state of availability of numerical models

- Standardize and document models chosen for use in support calculations

- Identify gaps in modeling ability, specific to YMP

- Extend existing models or develop new models

(4) Detailed Support Calculations

(a) Predictive Calculations

- Establish relevant scenarios, processes and parameter sets

- Generate computational results with adequate quantification of error bounds

(b) Design Monitoring System for Long-Term Validation

- Identify the suite of parameters necessary for long-term model validation

- Design an optimal monitoring system integrated with the proposed constructional and operational activities

3.3 Discussion

\subsubsection{Long-Term Evolution of the Yucca Mountain System}

The prediction of the long-term fate of the radioactive contaminants within Yucca Mountain is central to the approval of the licensing application. It is clear that the licensing application will have to rely heavily on predictive calculations using mathematical models related to fluid 
flow and chemical transport. Toward this end, much of the current emphasis is on collecting data on transport properties (e.g., permeability, porosity, desaturability, geochemical parameters of mobilization and retardation), spatial distribution of heterogeneities, and forcing functions (initial conditions, boundary conditions, sources), and to use such data as the basis for predictive modeling. This necessary and essential task has certain inherent limitations. Most of our experimental knowledge concerning these parameters is based on experiments that are of far shorter time scale (seldom longer than a few months) in relation to the time scale of predictive interest (millenia). Therefore our ability to make long-term predictions based solely on these data bases is limited. Moreover, our current data bases cannot provide us information on the long-term disposition of the forcing functions (e.g., rise or fall of the water table; extreme precipitation events). It is therefore essential that the modeling strategies that are currently being pursued be augmented by scmi-quantitative modeling ventures that address questions related to the hydrogeological evolution of the Yucca Mountain and its environs over the past 100,000 yr or so. A knowledge of the historical past should provide invaluable clues about the potential behavior of the system in the distant future.

This effort will broadly involve integration of hydrological, paleoclimatological and geochemical field observations as well as mathematical modeling. The geochemical studies would include study of the mineral assemblages in the vadose zone as well as their isotopic contents and the chemical characteristics of fluid inclusions.

\subsubsection{Flow in Heterogeneous Partially Saturated Systems}

The potential transport of contaminants by advective motion of the flowing fluids underscores almost all our concems regarding damage to the accessible environment. By definition, advection denotes the transport of dissolved or even suspended constituents by the bulk motion of the moving fluid. Therefore, a task of paramount importance in PA is to understand the macroscopic fluid flow field within Yucca Mountain. In a strongly heterogeneous system, the geometric pattern of fluid flow will be very complicated, being characterized by curvilinear flow paths that show pronounced patterns of convergence and divergence. These features of convergence and divergence have profound influence on flow velocities, chemical transport, and dilution and attenuation. Where flow lines converge, flow velocities will tend to increase and where they diverge, flow velocitics will decrease. Thus, the time of travel for a contaminant purely by advection is govemed by the genmetry of the flow system. Moreover, where flow tubes diverge, one should anticipate a tendency for attenuation of contaminant concentrations for two reasons. First, 
the greater volume of pore spaces avaiiable in the diverging flow paths will lead to relatively large dilution by mixing. Second, the slower velocities in zones of divergence will increase residence times and will abet fluid-solid chemical interactions.

There is thus a need to develop an adequate understanding of the type of fluid flow patterns that will characterize the Yucca Mountain system during the preoperative and postoperative phases. The special nature of heterogeneity at Yucca Mountain arises from: (1) megascopic heterogeneity due to the different units of layered tuff, (2) smaller scale variations of material properties within : riven lithologic unit, (3) the presence of micro- and macro fractures, and (4) the presence of fault zones and shear zones. To this list we may also add the spatial variations of transport properties that arise from the dependence of such transport properties on fluid phase saturation.

Very little is currently known from the literature about the nature of fluid flow in heterogeneous systems such as the one characterizing Yucca Mountain. Any insights that can be developed in this regard within the time constraints of the YMP nust be developed almost exclusively on the basis of numerical models. Even with the availability of supercomputers, it is doublful if the Yucca Mountain system can be studied in detail in three dimensions. Therefore, much of the insight and understanding that can be hoped for will have to be developed on the basis of carefully chosen two-dimensional studies. These conceptual investigations will contribute towards an understanding of the following topics:

- Lateral flows, perched water, transient migration of pulses, and evaporative fluxes induced by atmospheric variations in temperature and pressure,

- Generation of preferred pathways due to smaller scale heterogeneities, and the patterns of converging and diverging flow paths dictated by smaller scale heterogeneities,

- The implications of these flow geometries in regard to exirapolating transport properties from laboratory scale to field scale or in regard to generating transport properties from geometric information of rocks, and

- Design of critical experiments and monitoring systems.

\subsubsection{Nature of Evaporative Regime}

Within the YMP so far, much of the attention peraining to radioactive contaminant transpon at Yucca Mountain has been devoted to groundwater as the transporting agent. However, the issues discussed in the last chapter cleariy suggest that transport of contaminants by the gas $/$ apor phase could be very significant. Also, the transport of gases and water vapor within the mountain is intirnately related to the rate of infiltration of groundwater in the vadose zone 
Therefore, serious efforts need to be expended toward a clear understanding of the evaporative regime within the Yucca Mountain.

Recent field evidence (Weeks, 1987) from Yucca Mountain has clearly shown that active circulation of air within the mountain, driven by diumal and seasonal variations in pressure and temperature, may transport large volumes of air through the mountain through highly permeable pathways. Depending on humidity, this air circulation should be expected to transport large volumes of water vapor and gaseous constituents with time. In addition to the vapor movement induced by these air masses, vapor movement should also be expected in less permeable pathways, governed by other flow processes such as diffusion and film flow.

These studies on the evaporative regime will have to be conducted with the help of mathematical models, taking into account the flow of several fluid phases in a strongly heterogeneous system under nonisothermal conditions. The results of these studies will help evaluate the following:

- The magnitude of vapor flux from the vadose zone to the atmosphere on a long term basis,

- The role of the evaporative process in contuolling the downward infiltration of water,

- The potential influence of a strong evaporative regime on the long term decline of the water table, and

- The potential for contact with the waste package.

\subsubsection{Influence of Repository Thermal Regime on Evaporation and Infiltration}

The strong evaporative regime at Yucca Mountain which profoundly influences the hydrology of the vadose zone, will be even more accentuated by the strong thermal input from the decaying waste form. The temperature increase arising from this thermal input will probably peak about 100 yr after emplacement and gradually decline over several centuries thereafter. During the long period of thermal input, the movement of liquid water as well as water vapor will be greally modified in the vicinity of the repository. At the outset, one would expect the repository vicinity to undergo significant drying and loss of pore water. It is likely that this desaturation process will be dictated by a thermally-driven evaporative regime involving strong air convection as well as by buoyancy-driven liquid flow. If our present knowledge is any indication, even small differences of temperature and pressure are sufficient to maintain the strong cvaporative regime. It 6 . it is reasonable to infer that the anomalous evaporative regime induced by the thermal input of the uate package may periat for centuries. 
A credible evaluation of the induced evaporative regime following the emplacement of the wastes is essential for formulating and analyzing the many scenarios of contaminant transport that will form part of the licensing application. Here again, the conceptual and theoretical knowledge has to be generated with the help of mathematical models. This task will attempt to develop an understanding of the following:

- The geometry of the disturbed zone and its dynamic influence on the movement of fluids within the mountain,

- The influence of the disturbed zone on infiltration of water following extreme precipitation events, and

- The effects of large-scale desaturation and resaturation of the repository region on contaminant transport away from the repository.

\subsubsection{Interaction of Waste Package with Migrating Fluids}

Central to the rationale of the YMP is the notion of multiple barriers. These barriers include the waste form itself. The package will be designed to offer a minimum contact with groundwater when properly emplaced and will offer significant resistance to reaction with groundwater. The potential contact between the vadose water and the waste package will indeed determine the strength of the contaminant source. From the point of view of the source term, it is critical for the PA venture to investigate the relevant aspects of fluid-waste package contact and the associated possibilities of mobilization of the contaminant. Insofar as liquid water is concerned, this would include consideration of the orientation of the waste package with reference to flow geometry and the role of any diversion structures that may be designed to deflect liquid flow away from the waste package.

\subsubsection{Identification of Processes of Mobilization and Retardation}

Given a certain potential for contact between the fluids and the waste package, the contam. inants will have to be mobilized first from the chemically resistant waste form into the fluid phase (water, vapor or air) before they can begin their migration towards the accessible environment. Mobilization should be expected to occur by dissolution of contaminant chemical species in an environment of strong oxidation, augmented by the presence of acid-generating constituents such as sulfate, chloride and carton dioxide. The amount of contaminant mobilized will depend on the solubility of the particular constituent, its state of saturation, the nature and the state of the fluid phase, kinetic effects, and so on 
It is obvious that different chemical species will be mobilized at different rates. It appears that very few radioactive species (e.g. ${ }^{14} \mathrm{C},{ }^{129} \mathrm{I},{ }^{85} \mathrm{Kr}$ ) are potentiall $\mathrm{y}^{\prime}$ likely to be mobilized into the gas phase, while many more species could potentially get into the liquid phase. The task of identifying the particular species of imporance and the rates of their mobilization should take into account the chemical composition of the waste form, the nature of the waste package, and the nature of the fluids in the vadose zone. This is a critical task, as it dictates the nature of the source term.

Once mobilized into the fluid phase, the contaminants will be transported in the fluid phase by processes of advection, diffusion and dispersion along pathways that may eventually lead to the accessible environment. However, along the transport path, the dissolved species may also be demobilized or retarded from migration by various processes of fluid-solid interactions as well as radioactive decay. Specific retardation mechanisms of relevance in the context of Yucca Mountain are sorption, precipitation/coprecipitation, diffusion into stagnant pore-water, diffusion into the solid particles, and radioactive decay.

Quantitative evaluation of the chemical and physical parameters that govem demobilization of contaminants is a difficult task, particularly because predictions have to be made on a 10,000 yr time scale. Therefore, the necessary model parameters have to be evaluated and constrained using conventional laboratory experiments as well as careful analysis of field evidence related to the geochemical evolution of the Yucca Mountain system over the recent geological past.

\subsubsection{Evaluation of Relative Importance of Transport Processes}

The key transport processes that will enable the dissolved contaminants to reach the accessible environment include advection, molecular diffusion and hydrodynamic dispersion. Advection is the process by which transport occurs with the average bulk motion of the fluid phase. Diffusion is the process by which the contaninant is redistributed within the fluid phase as governed by concentration gradients. Hydrodynarnic dispersion is a process that is analogous to diffusion, in which the diffusion parameter is proportional to fluid velocity. Within the vadose zone at rucca Mountain, the ambient flux of liquid water is extremely small. Therefore, diffusion is likely to be the dominant transport mechanism, except when large fluxes of water occur, either along preferted pathways or due to strong infiltration pulses.

Clearly advection is the process that should cause the most serious concern, should rapid fluxes occur through preferted pathways (for example, saturated fractures, faults, shear zones). 
However, this concem must be evaluated in the broader context of the potential for mobilization of contaminants from the waste package.

In this broader context, it becomes very relevant not only to evaluate where the preferred pathways will exist with reference to the locations of the waste packages but also to know the duration over which the flowing water will be in contact with the waste form. It is clear that if water is channeled along pathways that are not intercepted by waste packages, then such pathways are indeed favorable features from the viewpoint of site suitability. Large transient fluxes along these pathways following extreme precipitation events will actually help drain the water quickly and maintain the long-term unsaturated regime. Furthermore, even if some of these benign pathways were to intersect some waste packages, the rapid movement of water will minimize the duration of contact between water and the waste package, leading to less sufficient chemical reactions and potential for mobilization (the magnitude of the contaminant source).

Thus the task of establishing the importance of advection within the Yucca Mountain is extremely important. Successful execution of this task entails the integration of issues related to fluid flow patterns in highly heterogeneous media, the response of the highly nonlinear system to extreme precipitation events, the ability of engineered barriers to divert water away from the waste package, the chemical interaction between transient fluid flow and the corrosion-resistant waste package, and the transport of mobilized contaminants downstream from the waste package.

This task is of considerable importance in relation to the regulatory notion of travel time. The notion of GWTT is well defined mainly in the case of a steady-state field of groundwater flow. In this case one may ignore, under conservative assumptions, the potential for retardation of the contaminant through fluid-solid interactions and compute the the time taken by a packet. of water to travel from the disturbed zone to the accessible environment along a flow tube. This simplistic notion cannot effectively take into account two important aspects of the basic rationale of the YMP, namely, rapid transient pulses of flux that may or may not intercept the waste package, and the duration over which the flowing water will be in contact with the waste package to cause corrosion and mobilization.

\subsubsection{Experimental Studies}

As pant of the site characterization effort, many activities are in progress, both in the field and in the laboratory. These ongoing activities need to be complemented by the following tasks of experimentation: 
- Measure unsaturated flow processes and parameters using osmotic gradients in the laboratory and in the field,

- Elucidate the process of water movement in the fractured tuff using modern techniques of three-dimensional imaging,

- Evaluate instabilities that may occur at sharp infiltration fronts in fractured porous media, particularly taking into consideration the finite mobility of air in heterogeneous media,

- Evaluate the nature of the moisture flow regime likely to develop around the waste package in response to the heat input, and

- Explore innovative methods to estimate the direction and magnitude of ambient insitu flux of water within the mountain.

\subsubsection{Verification of Numerical Codes}

The importance of code verification is well recognized in the PA endeavor and efforts are being made within the YMP to verify the different transport codes that are eventually expected to be used to generate computational results to suppon the licensing application. At present, the philosophy of verification manifests itself in two broad categories of activities.

The first is the testing of a numerical solution against a known analytical solution to a problem of interest. For the Yucca Mountain system, this philosophy of verification is of little practical use. The processes of fluid flow and transport within the mountain are governed by complex, interacting, nonlinear processes within a highly heterogeneous system. At present no known analytical solutions are available even for the simplest scenario involving these processes.

For want of anything better, therefore, the second philosophical approach of benchmarking is employed for verification. Benchunarking consists of setting up one or more hypothetical problems of the required degree of complexity and having these problems solved by several independent researchers employing independently developed numerical codes. The results generated by the various workers are carefully compared. If these results agree within reasonable limits, the codes are assumed verified and considered reliable for future use. If the results generated by the various workers differ markedly, then the cause of the differences must be examined and reconciled.

Although benchmarking is the best recourse currently available, there are strong reasons to look beyond benchmarking and to explore more credible ways of code verification, as pointed out by Narasimhan (1987). The physical processes of unsaturated flow are governed equations of state and motion. As the system changes its state, its storage of fluid changes in time. Fluid motion, which causes the change of state, is governed by the balancing of forces that impel the 
fluid and by frictional forces that resist fluid motion. The amount of fluid taken into storage and the energy spent in overcoming frictional resistance are both strongly governed by the nature of the flow geometry in a heterogeneous system. It stands to reason that the flow geometry will optimize itself in some fashion to minimize the energy spenit in overcoming resistance and the energy taken into storage. This perception of changes of state, causes of motion and optimization of energy expenditure is more elementary in nature than the classical differential equation and it involves direct consideration of integrals defined over the system of interest. Focusing attention on the evaluation of these integrals simultaneously with the determination of the most consistent flow geometry should lead to new and verifiable numerical algorithms that are of great practical interest to the goals of PA.

It is commonly understood that verification is the task of asserting that the numerical solu-

tion is internally mathematically consistent in regard to logic and accuracy. It is imperative to use verifiable codes in the PA venture for two reasons. The first obvious reason is that at the time of licensing hearings the credibility of the licensing application will be greatly enhanced by solutioris that can be shown to be verifiable. The second reason is more mundane and basic. Computational exercises already carried out within the YMP (and the erstwhile NNWSI) to develop conceptual insights regarding flow and transport within the Yucca Mountain vadose zone have generated alternative interpretations in regard to such important questions as perched water, lateral diversion of flow, and so on. These altemate interpretations have serious implications in regard to PA. In order to judiciously weigh these interpretations, it is imperative to identify and eliminate those aspects of the computations that are solely code-dependent and focus attention on the critical, intrinsic aspects of the system that are process-dependent.

For these two reasons, there is a strong justification to explore new ways of verifying the results generated by a given code in its own right, without requiring results from other codes, be they analytic solutions or numerical solutions. The ideal numerical code in this regard will be one which can be self-verifying (Narasimhan, 1987).

\subsubsection{Validation of Mathematical Models}

Model validation is the task of asserting that the processes simulated by the mathematical model indeed represent the physical processes that occur in the system of interest. In principle, the ideal validation is one in which the computed value of each physical variable is compared with the physically observed value of the same variable. Here again, much effort is being 
expended within the YMP and elsewhere within the DOE to validate mathematical models that will be eventually used in the PA. The task of validation is inherently much more difficult than that of verification. This difficulty arises in a large measure because of limitations in carrying out experiments, either in the field or in the laboratory.

The physical processes occurring within the Yucca Mountain involve the dynamic movement of more than one fluid (water, water vapor, air) in a highly heterogeneous system. While the mathematical models are capable of quantitatively evaluating many different physical quantities, the experimentally observable quantities are very few (e.g., fluid pressure, temperature and, occasionally, fluid saturation). Quantities such as fluid flux and fluid velocity are extremely difficult to measure within the porous medium. The difficulty is compounded by the fact that the measurable quantities are subject to scale of observation and the interruption introduced by the measuring instrument. In laboratory and field scale experiments, the possibility exists that the act of measurement itself may perturb the system.

At the present time, our ability to compute far exceeds our ability to conduct sophisticated experiments. It is almost impossible to physically measure all the various quantities that are computed by the mathematical model. Thus, complete and comprehensive validation of the vadose zone processes must be considered feasible only in the distant future. Within the context of the YMP, therefore, validation must be viewed in a more modest sense.

With this modest goal in mind, the task of validation may proceed along two mutually compiementary lines. The first of these is to design and conduct carefully controlled field and laboratory experiments, as is currently being attempted within such ventures as HYDROCOIN, INTRACOIN and INTRAVAL. Even at best, these experiments will be severely limited in scope in regard to our time scale of predictive interest, namely, $10,000 \mathrm{yr}$.

Considering the limitations of the conventional approach of direct validation of mathematical models through experiments, it is useful to explore alternate perceptions of validation in relation to YMP. In this connection it is appropriate to consider validation in the sense of mathematically simulating the hydrogeochemical evolution of the Yucca Mountain and its environs over the past $1(x),(X)(y)$ yr more. This type of validation will necessarily be a semi-quantitative exercise. However, it will attempt to integrate hydrology, geochemistry and climatology to draw broad inferences about fluid flow as well as chemical transport. These inferences may provide a per. spective on the potential behavior of the Yucca Mountain system over the next 10,000 yr which may not be allainable with the conventional validation ventures. This task of validation 
significantly overlaps the task discussed earlier in Section 3.3.1, Long Term Evolution of the Yucca Mountain System.

\subsubsection{Model Development and Extension}

As part of the NNWSI and the YMP, considerable efforts have been expended over the past decade to review, compile, verify and validate mathematical models which are likely to be used in the performance assessment of the Yucca Mountain. These efforts appear to have been motivated by an underlying notion that the PA venture will rely principally on existing mathematical tools, already available in the literature. There is merit to this perception because of the severe limitations of time and the voluminous computations that need to be carried out. Existing computational technology must surely form the backbone of the main computational infrastructure of YMP.

The many conceptual studies that have so far been carried out as part of NNWSI and YMP have indicated that the highly unsaturated, heterogeneous, fine-grained rock system within the Yucca Mountain introduces computational complexities that are not taken into account in general purpose computational algorithms already developed $n$. the literature. There is a critical need to expend a reasonable effort to modify and extend existing mathematical models to render them capable of addressing the special features of the Yucca Mountain system.

Perhaps the most distinct feature of the Yucca Mountain system in this regard is its heterogeneity in the context of unsaturated flow. Basic principles of laminar flow (which is nomally assumed to be representative of moisture movement in the vadose zone) suggest that the macroscopic pattern of water movement in such a system will be characterized by pronounced convergences and divergences of flow lines. The moving fluid encounters varying degrees of resistance along each flow path, depending both on the permeability of the materials encountered along the flow path as well as on geometry of the flow tubes as controlled by the convergences and the divergences. Because the flow resistance along each flow tube is a function of flow geometry, the pattems of flow resistance will change with the pattern of flow. It is reasonable to expect that the flow pattern existing at any given instant in time will reflect an optimal distribution of resistances as determined by the geometry of the flow pattern. Therefore, the fundamental goal of numerical modeling of the Yucca Mountain system should therefore be an accurate evaluation of this flow geometry. This statement applies to fluid flow as well as to transport, because fluid flow and transpon are governed by similar flow laws, namely, the laws of Darcy, Fick and Fourier. 
Although it is apparent that deciphering the flow geometry is a critical task of modeling the Yucca Mountain system, the conventional mathematical models so far used to analyze the system do not focus attention on this critical aspect. A majority (if not all) of the models used for analysis (models based on finite difference and finite element approaches) devote attention to approximating spatial gradients of potentials and concentration using as fine a grid as is practically feasible. The serious shortcoming of this traditional philosophy is that the accuracy of these approximations is seriously dependent on flow geometry. In order to ensure accuracy as well as verifiability of the solution, one has to use an arbitrarily fine discretization to be successful with these traditional models. In systems with ubiquitous small-scale heterogeneities (fractures, lenses) the required fineness of discretization may be unattainable for even two-dimensional problems at the present time.

Considering the limitations of the traditional models, there is sufficient motivation to extend or modify the traditional models in such a manner to enable them to focus attention on flow geometry. To achieve this it will be worthwhile to restate the governing equations of flow and transport by incorporating the flow geometry within these equations. It is reasonable to expect that such equations may lead to algorithms that are very appropriate to achieving the goals of YMP, both in regard to computational efficiency and in regard to verifiability.

\subsubsection{Predictive Calculations}

The ultimate goal of the PA effort is to generate calculations that will be incorporated into the licensing application. The successful accomplishment of this end entails the successful completion of two steps:

- the choice of a set of carefully chosen mathematical models, and

- the identification of appropriate scenarios and input parameters.

At the present state of the YMP it appears as though this task of predictive calculation must lag a little behind the other tasks. Although the currently available mathematical models will provide the broad computational infrastructure, it will be worthwhile to invest some effort into model extension and modification before embarking on the final predictive calculations.

Although the regulatory approach places a good deal of emphasis on predictive calculations bascd on ambient flow conditions, there is strong reason to believe that the ambient system will be markedly perturbed by the the thermal activity of the waste packages as well as by the large disturbances introduced by the underground mine openings and the ventilation systems. 
Therefore, it will be far more realistic to investigate the long-term behavior of the systern by giving consideration to expected postoperational perturbations than to rely exclusively on ambient conditions. At the present time we have very little knowledge of the possible postoperational system behavior to provide meaningful input for the predictive calculations.

\subsubsection{On-line Validation of Models and Monitoring}

Within the overall framework of YMP, the license approval process is but one of the major milestones. Because of the unusual complexity to characterize the natural system and because of the long time scale of prediction, the rationale of YMP includes open ends to account for unforeseen failures. Such incorporation of redundancies and open ends is part of the philosophy of any major project.

It is apparent that the licensing decision must be made on the basis of available data on ambient conditions and largely estimated information on system perturbations introduced by the construction and operation of the repository. It is inherently impossible to have concrete data on system perturbation until after the repository is constructed. Thus, the licensing decision will be based upon best judgement of system behavior, subject to the requirement that the system will be carefully monitored over the following several decades. During these decades, the system will be accessible to remediation measures and even removal of the wastes should unforeseen failures occur. This open-endedness is a particularly favorable aspect of YMP and provides strong backup support for the licensing decision.

If successfully implemented, monitoring of the system during the construction and operation of the repository will provide a data base that will probably be unmatched in any comparable project; we currently know of no engineered system that can boast of a reliable data base even for a decade. The nature of the wastes and the fluid flow system within the mountain are such that system failure may not be catastrophic and that remediation could be implemented over a sufficient period of time. Finally, should new technologies become available within the coming decades that would reinforce the natural barrier within the mountain (e.g., introduction of biological retardants into the system), such technologies can be advantageously used within the mountain.

The final task in PA venture is thus to design a monitoring systern that will evolve with the project and will continue to be operational well into the postclosure period. This task too, just as the lask on predictive calculations, will have to be initiated after the completion of other tasks which provide the necessary input for designing the monitoring system. 
During the active phase of the repository, the monitoring activities will be supplemented by ongoing validation activities. Idealiy, the validation activities will lead to reliable, well-calibrated models that will help define the final site-closure plan.

\subsection{Sequencing of Tasks}

In the light of the foregoing discussions, we may attempt a broad prioritization of tasks into three phases. The specific content of each task will need to be worked out in detail along lines suggested under the discussions on issues and on tasks needed for issue resolution.

Phase 1 Hydrogeochemical evolution over recent geological past

Nature of evaporative regime

Laboratory and field experimentation

Evaluation of essential processes of fluid flow and transport

Verification and validation of models

Model development and extension

Phase 2 Flow in heterogeneous unsaturated systems

System perturbation because of repository construction and operation

Interaction of fluids and waste package

Phase 3 Detailed predictive calculations

Design of monitoring system 


\subsection{A SUMMARY OF FINDINGS: $1983-1989$}

Thus far the focus of our unsaturated flow project with SNL has been on understanding the mechanisms and on modeling the hydrological processes in partially saturated, fractured, porous tuff at Yucca Mountain. We started with a spatial resolution in the submillimeter range for the discrete fractures and extended consideration to kilometer scale for an east-west crosssection of Yucca Mountain. The time scale of interest ranged from minutes for the desaturation of fractures to thousands of years for infiltration through the partially saturated zones. The objective of performance assessment of the hydrological processes at Yucca Mountain is to predict the threedimensional responses of the whole system from the repository to the accessible environment over thousands of years. Starting with the small scale, this project has gradually evolved to the large scale which is of ultimate interest for performance assessment. The broad pattern of this evolution can be summarized as shown in Figure 4-1.

\subsection{3: Conceptual Model for Moisture Movernnent in Fractured, Porous Tuff}

During 1983, the first year, we focused on the develc pment of a conceptual model to understand the hydrological mechanisms governing fluid flow in a partially saturated, fractured porous medium. The Topopah Spring welded tuff formation is highly fractured with tuff matrix blocks of sub-meter sizes surrounded by discrete fractures. The main question we addressed was whether water moves in the fractures or in the matrix under partially saturated conditions. This is one of the most important hydrological questions in determining the flow pattern and travel time through fractured, porous units. This single question will remain as one of the key issues to be resolved and quantified throughout the course of YMP investigations.

\subsubsection{Capillary Mechanism: Preferential Saturation of Fine Pores}

We recognize from the onset of this project that fractures with large apertures will drain relatively easily and water is likely to remain in the matrix. This intuition stems from the knowledge that large pores can not sustain large capillary suction forces. In a heterogeneous system where sand and clay coexist, the sand with large pores can be easily drained and the clay will remain wetter than the sand under partially saturated conditions. This can be readily explained by the nature of the capillary mechanism. When we insert a capillary tube into a beaker of water, the height of water rise along the capillary tube is inversely proportional to the radius of the tube. 
Conceptual Model for Moisture Movement in a Fractured Porous Tuff<smiles>[CH][CH]</smiles>

Derivation of Functional Dependences of Unsaturated Transport Properties<smiles>[CH][CH]</smiles>

Vertical Infiltration through a Single Column<smiles>[CH][CH]</smiles>

Response of a Vertical Column to Extreme Precipitation Events<smiles>[CH]1CC1</smiles>

Moisture Movement in a Two-Dimensional, East-West Cross Section<smiles>[CH]C</smiles>

Constraints on Unsaturated Transport Properties and Application of Fractal Theory

Figure 4-1 Evolution of the hydrologic performance assessment task. 
A dry towel with fine texture can soak up more water than a rag with coarse pores. With capillary theory, we anticipate that the larger pores will desaturate more easily than smaller pores. If this simple capillary model is valid, we can easily infer that fractures in a fracture porous medium will tend to reriain ary under partially saturated conditions and the liquid water is held by capillarity in the finer pores of the matrix.

Before we describe the response of fractured tuff with capillary theory, we must point out that the capillary mechanism has a limited range of validity. If the radius of the capillary tube or the aperture of the parallel plate fracture is large, the radius of curvature of the meniscus will be infinite and the capillary effect will be negligible. From the surface tension of liquid water, the maximum capillary size is estimated to be approxirnately $0.4 \mathrm{~mm}$. For fracture aperture and macropore radius greater than this size, a capillary-based conceptual model is not valid. In capillary theory, we ignore the small fraction of water molecules adsorbed by the solid wall above the meniscus. The slow movement of adsorbed water outside the liquid region may be important in extremely dry conditions. The capillary limit and adsorptive mechanism are two hydrological issues which may be imponant for studying the validity of capillarity-based conceptual model.

\subsubsection{Natural Fracture Model: Importance of Wall Roughness}

In quantifying the capillary mechanism in fractured tuff, we recognize that the parallel-plate model frequently used in saturated fracture flow studies is too crude and simplistic to represent natural fractures under partially saturated conditions. In a highly fractured formation such as the Topopah Spring welded tuff unit, the fractures divide the tuff matrix into blocks surrounded and bounded on all sides by the fractures. If the fractures are represented by parillel plates, then the fractures will either be completely filled with liquid water or be completely drained. Liquid water within the porous blocks, isolated by the drained fractures, cannot flow from one matrix block to another. With parallel-plate fractures, liquid water in the matrix blocks cannot move and travel time will effectively be infinite under partially saturated conditions. This is simply too idealistic a model for performance assessment of flow and transport through a partially saturated, fractured porous formation.

Natural fractures are characterized hy rough surfaces. In fractures at depth under compressional stress, the walls are pressed together, with a finite fraction of fracture surfaces in contact, such conacts being known as asperities. The aperture of a fracture will likely be smaller near the asperties and larger in the open channels within the fracture plane. As a fracture desaturates, the 
open region with larger apertures will be drained easily and liquid water will remain as pendular islands around the contact areas. The wetted regions centered arourd the contact areas provide the bridges for liquid water to flow across the fracture plane from one matrix block to its neighboring matrix blocks. Two schematic figures are used to illustrate the flow pattem and fluid distribution in a fractured porous medium. Figure 4-2 is in a cross section cut normal to the fracture plane showing the pores in matrix blocks and fractures with spatially varying apertures. Figure 4-3 looks down on a fracture surface showing the distribution of contact areas and fluid configurations. With portions of fractures dry, liquid will tend to flow across fractures from one matrix block to another instead of flowing along the fractures. One may expect the flow lines to circurnvent dry portions of the fractures as illustrated in Figure 4-2. As a fracture desaturates, the liquid phase configuration will change from continuous phase at high saturation to discontinuous phase at low saturation with liquid forming rings around contact areas. With the idealized array of contact areas in Figure 4-3, a sharp cutoff transition will separate the continuous liquid phase configuration from the discontinuous configuration.

Capillary theory, together with the rough fracture model, forms the basis of our conceptual medel for a fractured porous medium under partially saturated conditio..s.

\subsubsection{Aperture Distribution: Approach to Quantify a Rough Fracture}

Mathernatically, a rough fracture may be characterized by an aperture distribution function. We have derived relationships for fracture saturation, fracture permeability, and fraction of wet fracture surface as functions of fluid pressure in terms of integrals over the aperture distribution function. The general relationships can be used with any aperture measurement and distribution function. The detailed derivations and results to quantify the conceptual model are given in our first report (Wang and Narasimhan, 1984) and an archival paper (Wang and Narasimhan, 1985). For one simple aperture distribution function, the gamma distribution, we integrated explicitly to obtain algchraic relationships for the fracture characteristic curves.

The gamma distribution function, $n\left(b_{0}\right)=\beta^{2} b_{0} e^{-\beta b_{0}}$, has only one parameter $\beta$ to describe the variation of aperture $b_{0}$ of a fracture under zero stress. Under zero stress, the fracture surfaces are in point contact and the contact area is zero. In practice, $n\left(b_{0}\right)$ can be measured by scanning the open fracture surfaces and tracing the roughness profiles. The mismatch between the roughness profiles between two surfaces yields the aperure distribution function. To this date, very litthe data have been available to quantify the aperture distribution function for tractures in yucca 


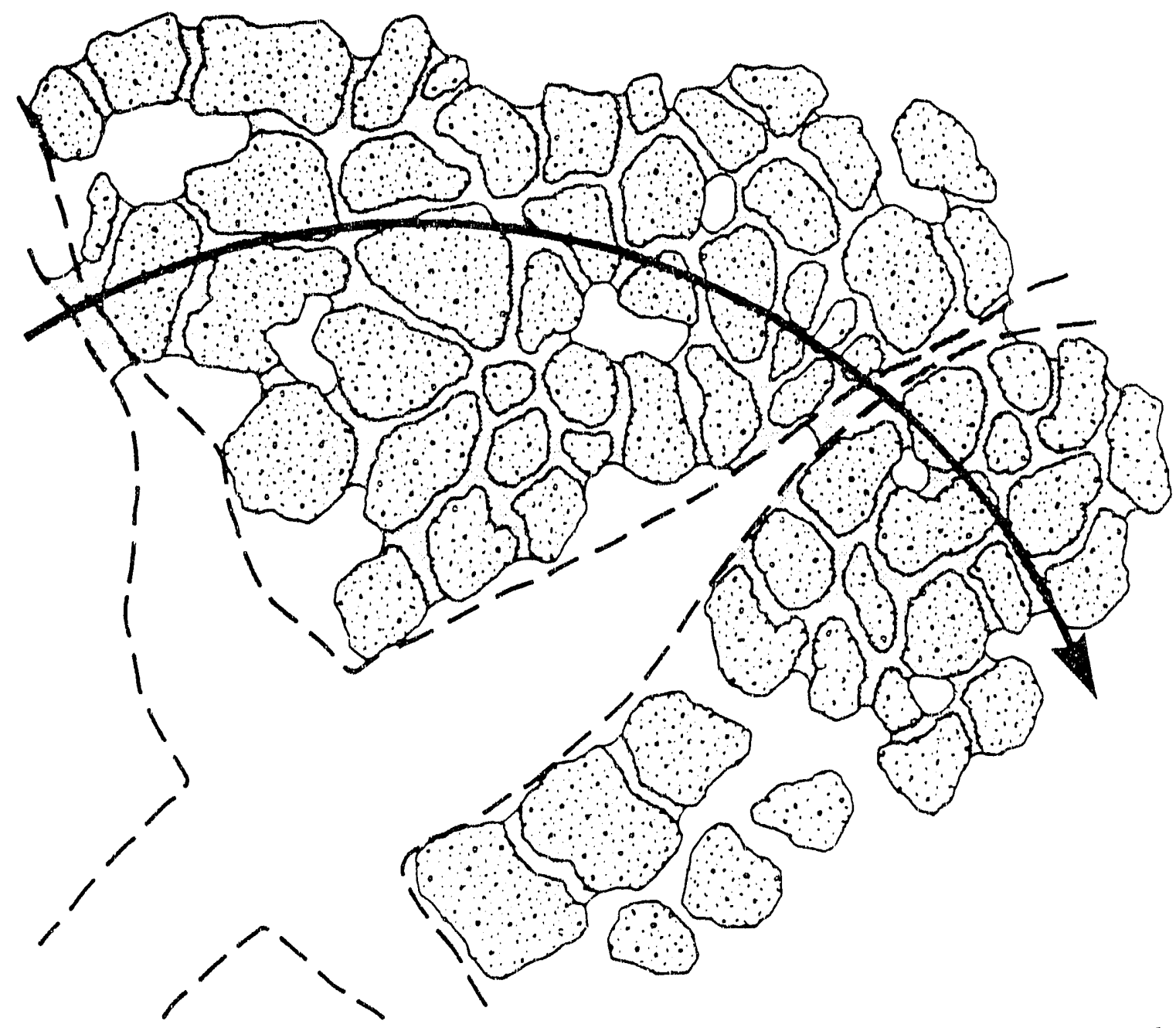

XBL $841-9580$

Figure 4-2 Schematic representation of water flow in a fractured porous medium. 


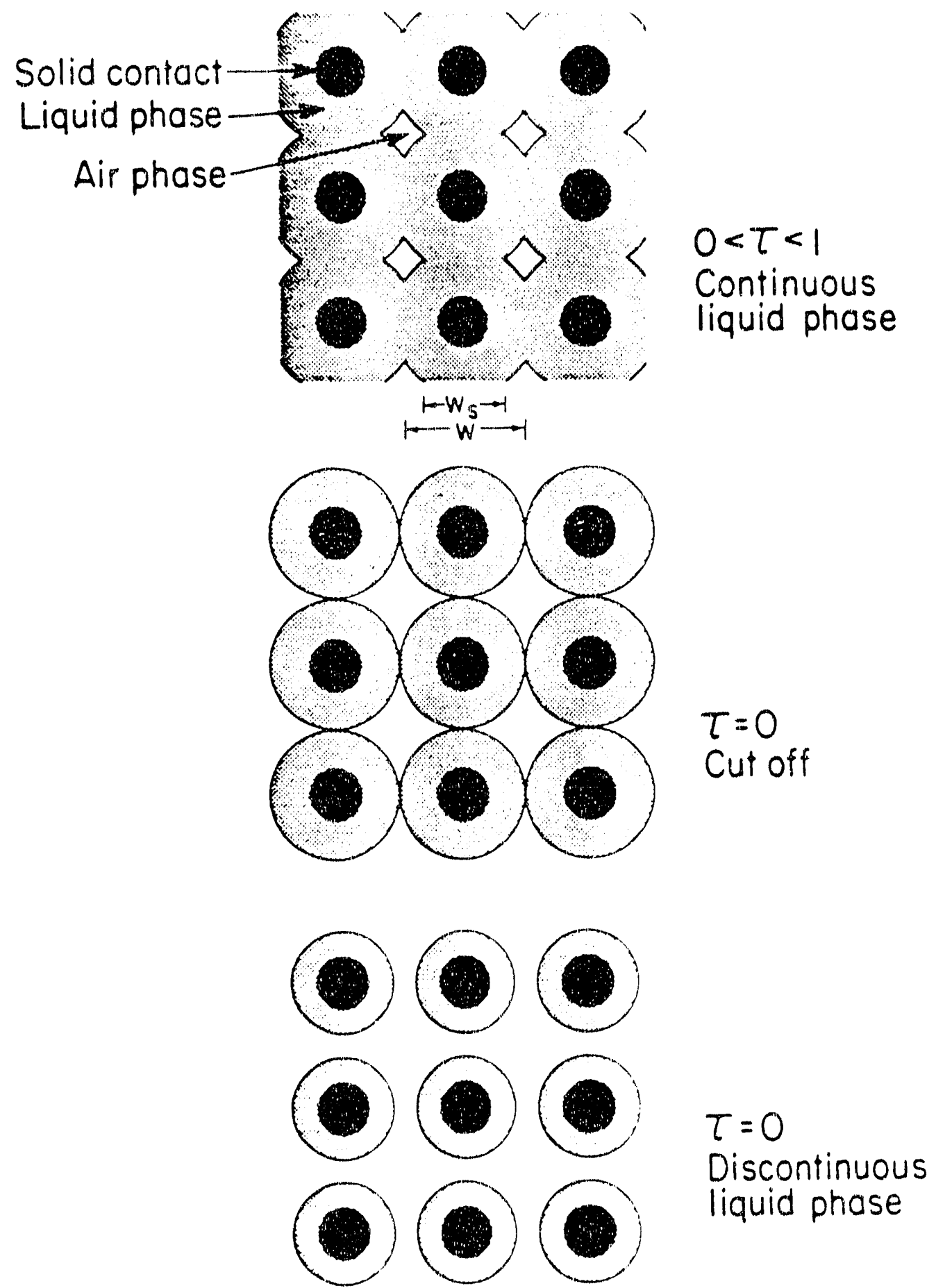

$x 81842-9615$

Figure 4-3 Schematic plan view of a fracture plane showing asperities (solid circles), liquid water (gray) and air (blank). 
Mountain tuff. The simplicity of the gamma distribution function and especially the fact that there is only one parameter to quantify were on our minds when we chose it as the basis for detailed study. An added reason was that the limited analysis available at that time from a French study of natural granite fractures by Gentier (1986) indicated that $n\left(b_{0}\right)$ takes on a skewed shape, with long tails toward large apertures (Tsang, 1984). The gamma distribution can fit these aperture measurements well.

We should emphasize that we did not use granite data to represent tuff fractures. We used the gamma distribution function only to represent the functional form of the aperture distribution. Without actual aperture measurements on fractures in tuff, we used available fracture spacings and bulk permeability of tuff to inversely determine the $\beta$ paramettrs. The assumptions and procedures used to determine $\beta$ parameters from available tuff data were discussed in our first report. All the parameters used in our simulations were derived from available tuff data and no other parameter values were assumed or adjusted in the tuff fracture desaturation curves.

At Yucca Mountain, there are two nearly vertical fracture sets: one strikes roughly northnorthwest, and the other, north-northeast. Fracture inclination data from borehole USW-G4 (Spengler and Chomack, 1984) also indicate the presence of a minor, nearly horizontal fracture set. The fracture distribution functions derived for the vertical sets (assumed to have equal parameters) and the horizontal set are plotted in Figure 4-4, with distribution parameters derived from Topopah Spring welded tuff data. The distribution functions are normalized, with the areas under the curves set equal to unity. Both vertical and horizontal fractures have hydraulic apertures in the sub-millimeter range according to these indirectly derived aperture distribution functions.

\subsubsection{Flow Across Fractures: Importance of Effective Flow Area}

For fractures underground, we expect that a fraction of the apertures will be closed under stress. We do not have data for the fraction of in situ contact area. However, borehole USW-G4 core analyses indicate that about $12 \%$ of the fracture surfaces have coatings of zeolite, clay, and calcite which are likely minerals precipitated from water. We made an assumption that the fracture coatings of $12 \%$ correspond to the fraction of in sits contact area. We assume that contact areas remain wetted for a long time and allow hydrochemical alterations to occur in contact areas for the coatings 10 form. This assumption to relate fracture coatings with fracture contacts remains to be checked for consistency against other goechemical and geomechanical data. We 


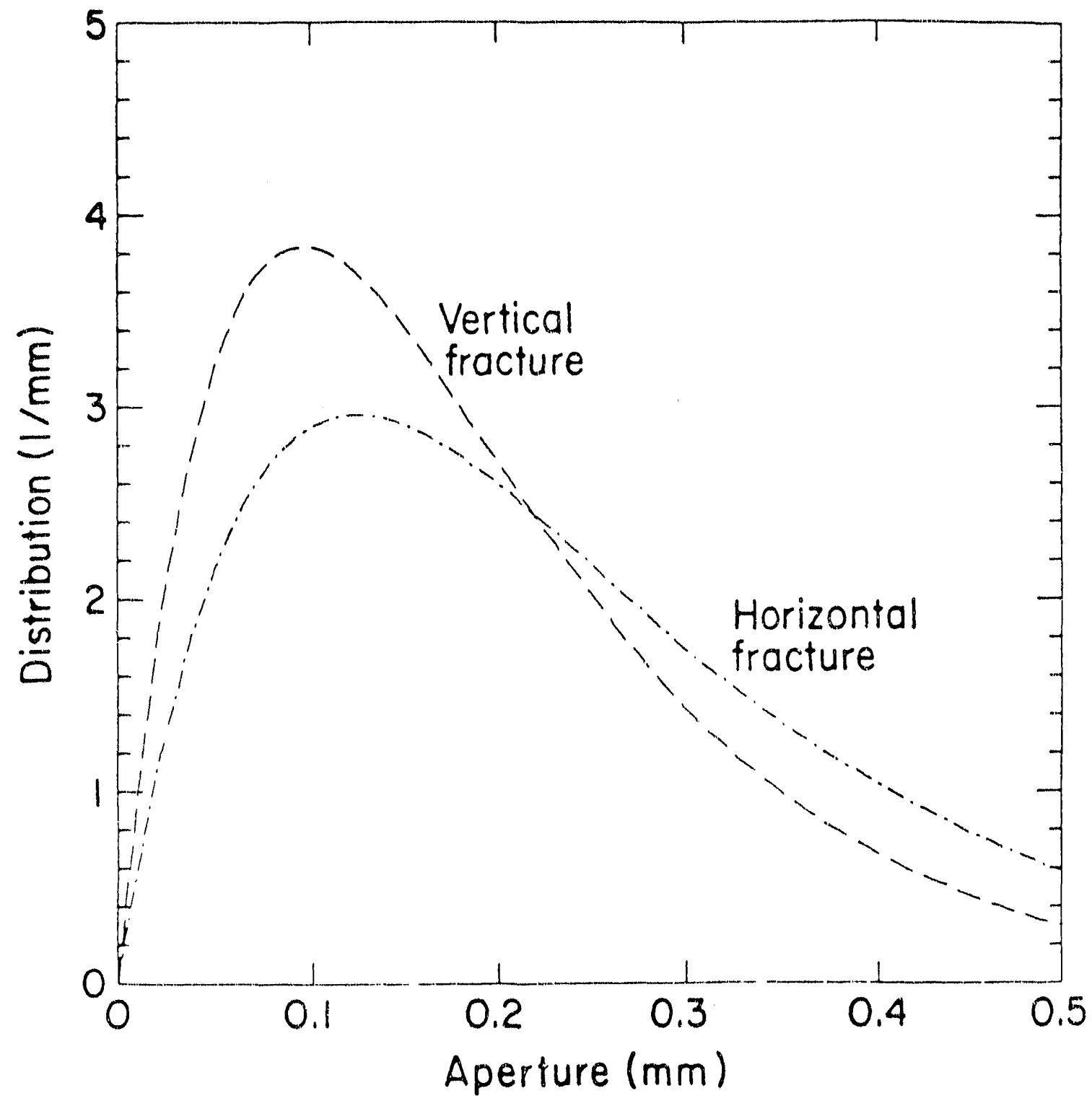

XBL $842-9616$

Figure 4.4 Aperture distributions for the Topopah Spring welded unit. 
believe that the evolution of fracture coatings can shed light toward a better understanding of the long-term processes relating mechanical deformation and stability, chemical dissolution and precipitation, and hydrological flow and transport in fractured, porous tuff under partially saturated conditions.

If both the matrix blocks and the fractures are fully saturated with liquid water, the whole fracture-matrix interface will be available for water to flow from the matrix into the fracture and vice versa. As the fractures drain, only a fraction of the area in the fracture surfaces will enable flow from one matrix block to another block across fracture. Figure 4.5 shows that the effective fracture-matrix flow area at fracture-matrix interfaces decreases and quickly approaches the limiting contact area fraction of $12 \%$ as the pressure head decreases. With liquid flow from one matrix block to another restricted to cross the interfaces through reduced areas, the flow lines will converge toward, then diverge away from contact areas. Therefore, flow paths will be more tortuous in a partially saturated, fractured porous medium. The pressure dependence of effective fracture-matrix area is not taken into account in conventional numerical models. We incorporate this effective area dependence into the TRUST code (Narasimhan et al., 1978) to properly simulate the flow in fractured tuff systems.

\subsubsection{Transport Properties of Fractured Tuff: Derivation of Functional Relations}

The relation between water saturation and pressure head and the relation between permeability and pressure head are two physical functions that are essentially required for modeling water movement in the fractured tuff. Using the gamma function for aperture distribution, and using principles of capillary theory, we have derived expressions from these functional relationships. Figure 4-6 compares the fracture characteristic curves with the tuff matrix characteristic curve of saturation versus head for the Topopah Spring welded unit. The tuff matrix curve was provided by SNL from laboratory measurement of a representative core sample (Peters et al., 1984). The themocouple psychrometer matrix data were fitted with the equation suggested by van Genuchten (1980). Figure 4-6 shows that the fractures will desaturate easily with a small suction of -f). $1 \mathrm{~m}$. The saturation in the matrix. will remain high. If the in situ matrix saturation in Topopah Spring welded unit is 0.8 , then the negative pressure head in this unit is over $-100 \mathrm{~m}$. With such a large suction, the fracture saturations will be essentially zero.

The relations between effective permeability and pressure head are shown in Figure 4-7 which shows that the fracture permeabilities are very sensitive to suction. If fully' saturated, the 


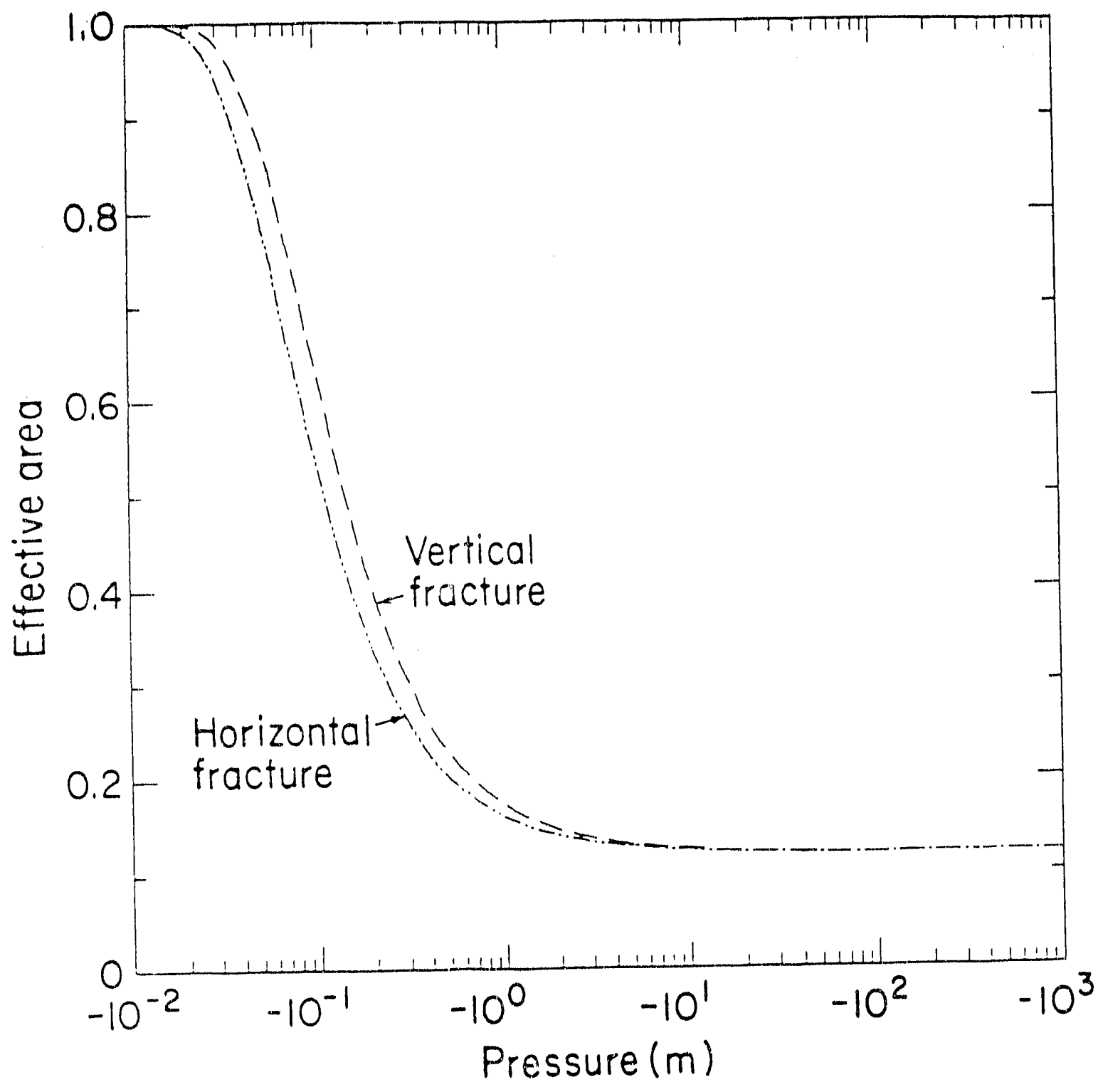

XBL 842-9621

Figure 4-5 Effective flow area versus pressure head for flow across the fracture plane. 


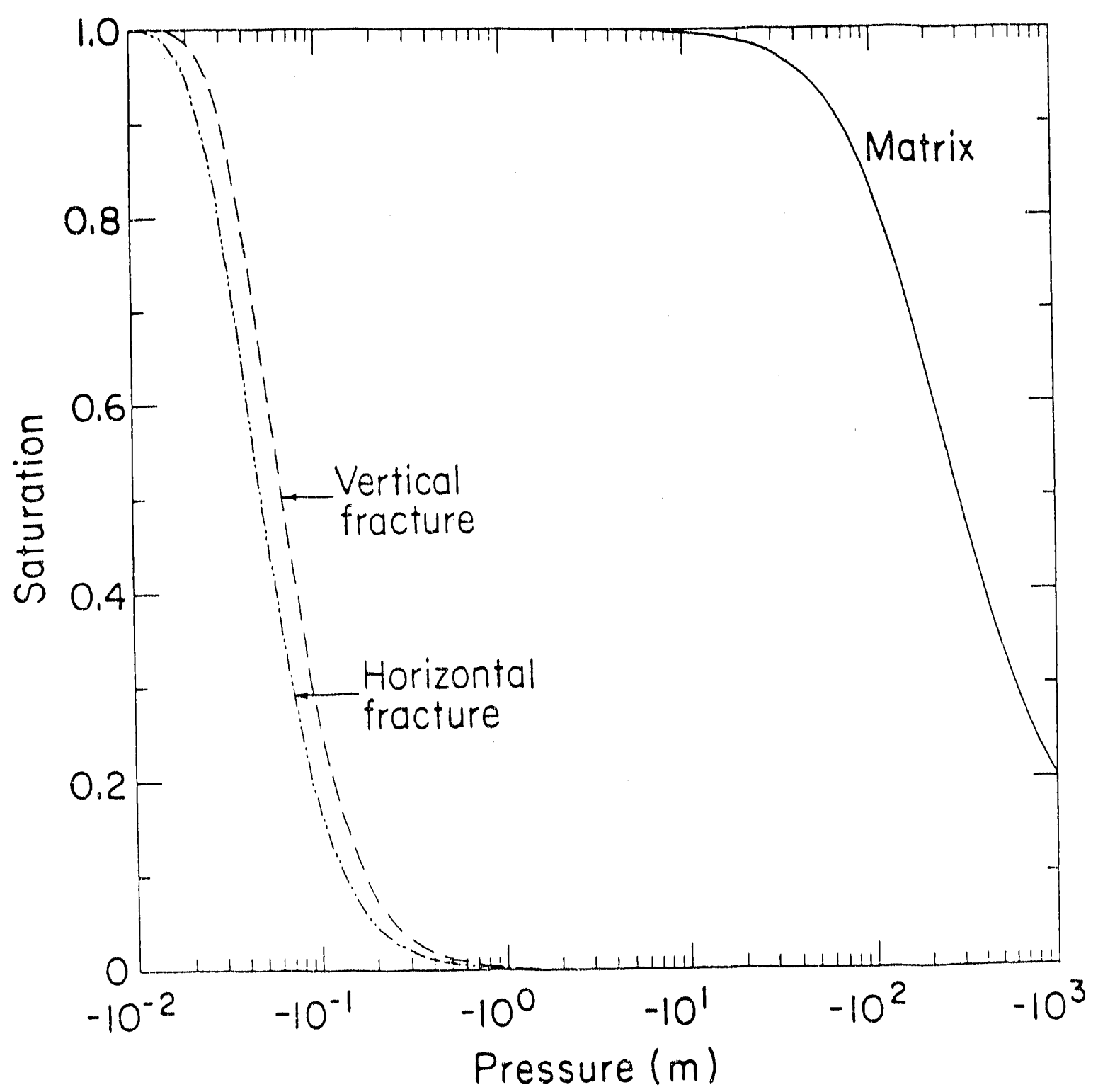

X8L $842-9617$

Figure 4-6 Saturation versus pressure head for a rough-walled fracture. 


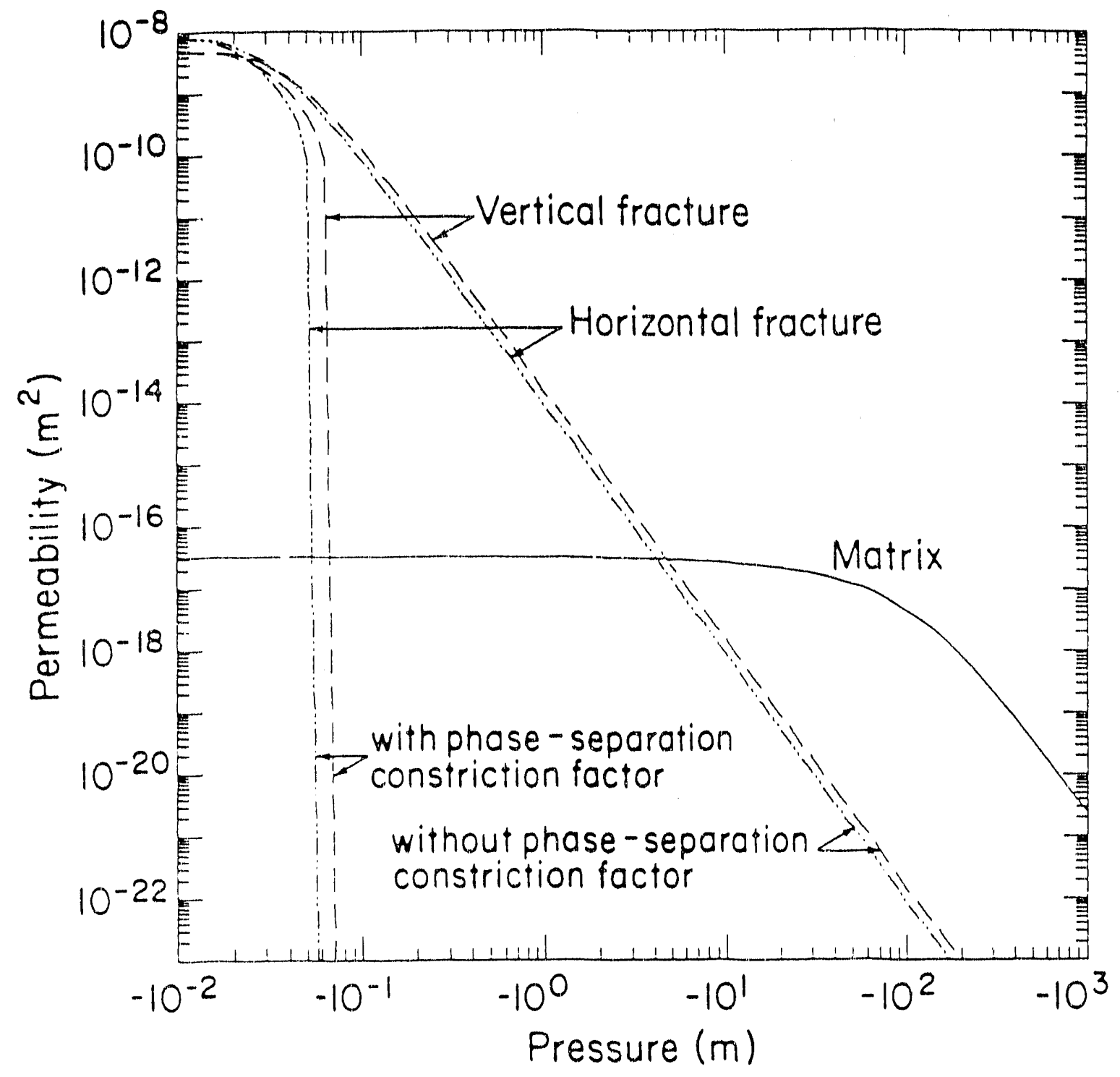

$X B L 842-9620$

Figure 4-7 Effective permeability versus pressure head for a rough fracture. 
permeability of each discrete fracture is eight orders of magnitudes greater than the matrix permeability. With small suctions in the range of $-0.110-10 \mathrm{~m}$, the discrete fracture permeabilities decrease drastically. The matrix permeability decreases much more gently as the pressure head becomes more negative. Near fully saturated conditions, the fractures will control the fluid flow. As desaturation proceeds and the fracture permeability of each discrete fracture becomes less than the matrix permeability, the matrix will control the flow. With in situ suction of over $-100 \mathrm{~m}$, fracture flow diminishes and matrix flow dominates. The drastic change of the role played by the fractures as the active main conduits for flow and transport in saturated conditions to become passive dry pores in unsaturated conditions is the most important result arising from our conceptual model. If the role reversal between fracture flow and matrix flow does not occur under ambient partially saturated conditions, then the highly fractured tuff unit will transmit liquid water very quickly through the connected fracture networks and the travel time will be very short. Substantiating our finding for the role reversal between fracture flow and matrix flow is crucial for the performance assessment of the partially saturated flow field at Yucca Mountain.

\subsection{4: Drainage Responses of a Fractured Tuff Column: Dynamic Interaction between Discrete Fractures and Porous Matrix}

With the development of a conceptual model based on capillary theory and aperture distribution of rough-walled fractures, we used the fracture characteristic curves derived in 1983 to study the detailed responses of a fractured, porous tuff column, taking explicitly into account the vertical and horizontal fractures and tuff matrix blocks. Our finding that fracture pressure and matrix pressure are approximately equal after the column is drained provides a rational basis for the development of a composite medium model at SNL by Peters and Klavetter (1988). In addition, the dominance of liquid flow in the matrix, together with the findings by Pruess $e t$ al. (1985) that gas flow occurs mainly through the fractures has provided incentive for the concurrent development of a multi-phase composite medium model. This model is known as an effective medium model. Detailed studies with discrete fractures are valuable for better understanding of system behaviors and for developing useful approximations for large-scale analyses. 


\subsubsection{Fracture Description: Adaptation of Field Data}

To construct the detailed model representing the Topopah Spring welded tuff unit, we first analyzed available data on fracture orientation and spacing from borehole USW-G4 (Spengler and Chornack, 1984). By grouping the fractures as either nearly vertical or nearly horizontal according to their dip inclinations, and using the geological observations that there are two nearly vertical fracture sets (Scott et al., 1982), we determined the average spacings of the fracture sets as shown in Figure 4-8. We assumed that the two nearly vertical sets had equal spacing and that they contributed equally to the equivalent fracture continuum permeability (cube of aperture divided by spacing).

Without the direct availability of data for the equivalent fracture continuum permeability in 1984, we deduced the equivalent permeability value from well testing in well $\mathrm{J}-13$ (Thordarson, 1983). Well J-13 is located in a low-standing structural block east of Yucca Mountain with part of Tupopah Spring welded tuff unit below the water table. It is assumed that the water pumped out during testing had moved through a connected network of fractures which provide effective pathways for fluid movement under saturated conditions. We also assumed that equivalent fracture continuum permeability was isotropic. All these simplying assumptions about the fracture network can be relaxed easily when additional and more detailed field data become available. In the course of this project, we have indeed continuously updated our models as more realistic data were provided by SNL and incorporated them into the later simulations. The procedures outlined in our first report on fracture network analyses are straight forward and can be easily generalized to determine anisotropic fracture spacings and non-parallelepiped blocks from more data on anisotropic equivalent continuum permeability tensors.

\subsubsection{Discrete Fracture - Porous Matrix Model}

With the spacings of the fracture sets and the sizes of the tuff matrix blocks determined, we constructed a small model to simulate vertical drainage of a fractured tuff column (Figure 4-8). Two horizontal fractures, normal to the direction of general flow, are explicitly simulated. On the local scale, lateral flow is allowed between the vertical fracture and the matrix blocks. The horizontal cross section of a matrix block is further partitioned into six nested elements and the vertical cross section of each block is sliced into nine sections. The grid elements are small near the fractures and large toward the middle of the matrix block. The partition of horizontal nested elements is similiar to that used in the multiple interacting continuum idealization (MINC) (Pruess 


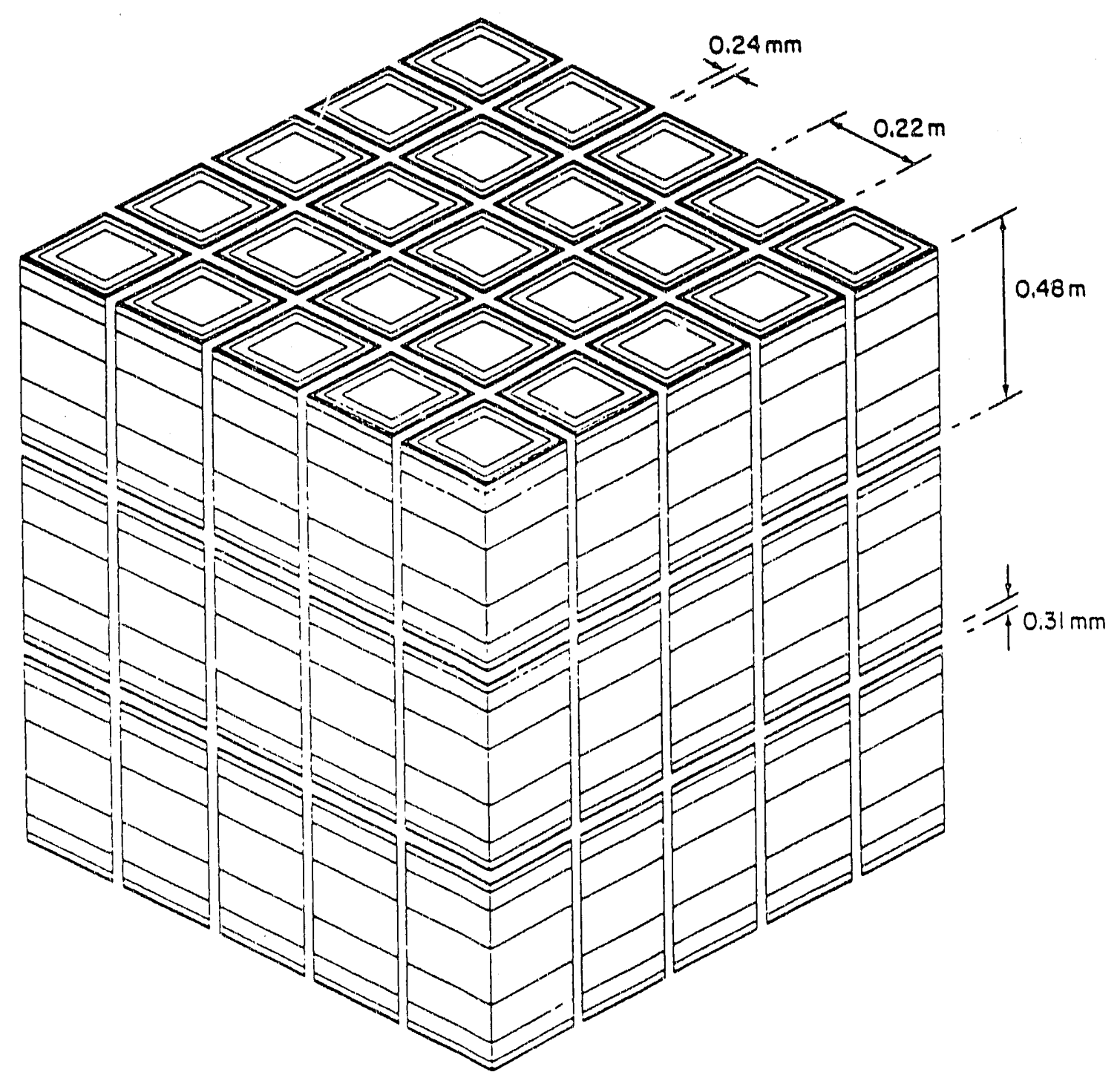

XBLB42-9614

Figure 4-8 Schematic representation of three fracture sets for the Topopah Spring welded unit. 
and Narasimhan, 1985). Such a nested grid allows the simulation of convergent flow from the fractures into the matrix block and divergent flow from the matrix block into the fractures with relatively few elements if we are interested mainly on the fracture-matrix interflows and not concerned with the detailed flow field near the diagonals within the matrix block. The nested elements also take properly into account the geometrical configuration with each horizontal cross section of a matrix block surrounded by fractures on all four sides. The usual semi-analytic treatments of fracture-matrix flow with one-dimensional approximations do not properly account for the multiple-side effects. In the simulations, we did not group the matrix elements from different blocks at different elevations together. The two horizontal fractures and the three matrix blocks within each vertical column are explicitly simulated. In this respect, we did not carry out the simulation in the multiple interacting continuum grouping approximation and account for the effects of discrete fractures in detail.

\subsubsection{Tool of Analysis: The TRUST Code}

In 1984, we modified the TRUST code to allow the available area for flow across the fracture-matrix interfaces to be determined by the fracture saturations. At the limit that the fractures are completely desaturated, the available area is the contact area between adjacent blocks for matrix flow from one block to the next. The outermost matrix node for one block is connected to the fracture and is not directly connected to the matrix node in the next block in the simulations. It is important to note that fracture permeabilities are intrinsically defined only along the fracture planes. Normal to the fracture matrix interfaces, the fractures will not introduce any resistance under both the saturated conditions and under partially saturated conditions with flow through finite wetted areas around contact points. With the integrated finite difference method (Narasimhan and Witherspoon, 1976) in the TRUST code, this can be easily taken into account by setting the distances from the fracture nodes to the fracture-matrix interfaces to zcro so that normal flow from matrix blocks to fractures is controlled by matrix permeability. We point out this detail in the simulation because the conventional finite difference and finite element codes do not usually take this intrinsic anisotropy of fracture permeability into account. If the proper treatment of fracture permeability is not taken into account, the zero permeability of the fracture elements under completely desaturated conditions will block the flow from one matrix block to the next. Physically, we expect that flow will cross one matrix block to another matrix block through the finite contact areas. 
In 1984, we used the usual hamonic mean approximation to determine the conductances at the element interfaces from perneability values of neighboring nodes weighted by the distances from the node center to the interfaces. If we use the geometry-imbedded Darcy's law (Narasimhan, 1985), we can probably reduce further the number of nested elements from six to one or two for each horizontal matrix block cross section and similiarly for the vertical grids. The development of geometry-imbedded path-integration modeling techniques can increase the efficiency and accuracy of modeling discrete fracture-porous matrix systems. This is a promising task to be investigated further.

\subsubsection{Major Findings: Flow Dominance by Matrix.}

We simulate the transient changes in the fractured tuff column from initial fully saturated condition to final parially saturated condition. Iritially the column is filled with water with pressure determined by hydrostatic equilibrium. The hydraulic head is everywhere zero and fluid is stationary. At time $\mathrm{t}=0^{+}$, a negative suction head is applied to the lower boundary to induce drainage and transient changes in the fluid flow field throughout the fractured, porous tuff column. The boundary suction head is over $-100 \mathrm{~m}$ which corresponds to a final ambient matrix saturation of $S=0.8$.

The transient pressure drops at different locations in the column are shown in Figure 4-9 for three cases. We studied five cases for flow in the column: (1) a fractured, porous tuff matrix colurnn with the phase-constriction factor, $\tau$ (Wang and Narasimhan, 1984) taken into account for the discrete fractures $(1 \leq \tau(h) \leq 1)$; (2) a fractured, porous column without the phase-constriction factor $(\tau=1)$; (3) a matrix column without fractures; (4) a discrete fracture column without matrix and with $\tau$ factor; and (5) a discrete fracture column without matrix and without $\tau$ factor. The locations of interest are the middle of the matrix block (point $A$ ), the middle of the vertical fracture (point $B$ ), the middle of the upper fracture (point $C$ ), and the middle of the lower horizontal fracture (point D), as shown in the inserts of the plots.

The pressure drops for the first three cases at different locations in Figure 4-9 are essentially the same after an initial desaturation period of $10^{-2}$ years. Within this short period, the onset of fracture desaturation depends on whether the phase-constriction factor is taken into account as shown in the small differences between the top two plots in Figure 4-9. As pressures become negative folk wing initial desaturations, the pressure changes are controlied mainiy by the matrix. At the same clevation, the matrix pressure at point $A$ and we vertical fracture pressure at point $B$ 

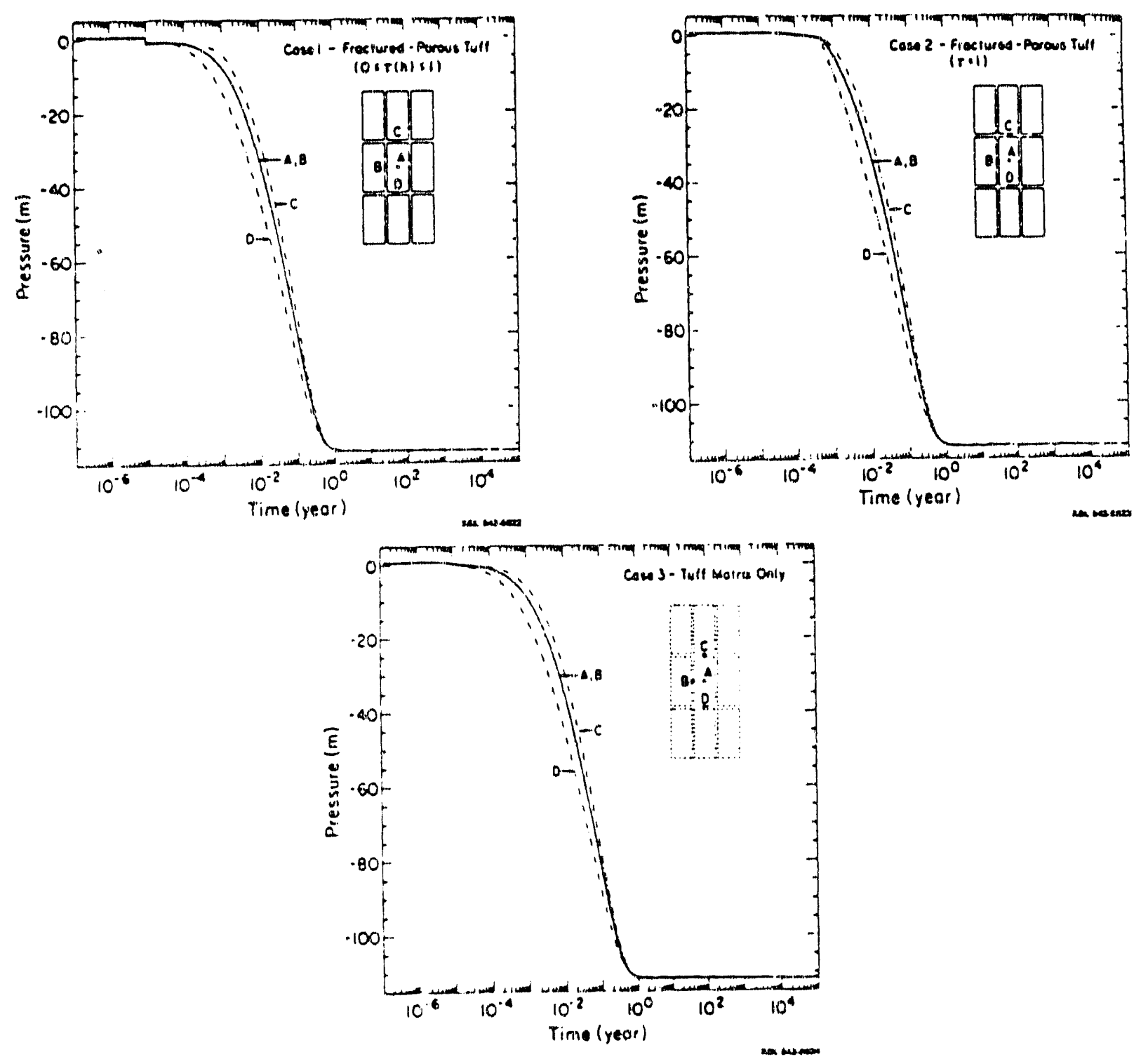

Figure 4.9 Unsaturated drainage in a tuff column: comparison of results for three different concentualizations 
are approximately the same. The lower fracture, point $\mathrm{D}$, is closer to the suction boundary and has more negative pressure than the higher elevation points. The simulation results involving only matrix in the third plot in Figure 4-9 are nearly identical to the results with discrete fractures simulated. It is clear, therefore, that pressure changes can be simulated with matrix properties only, if the transition from saturated conditions to unsaturated conditions is not of interest. We also simulate the changes in the fractured column without the matrix (case 4 and case 5) and obtain very unrealistic results to illustrate the importance of porous matrix blocks as flow channels for fluid to change pressure (Wang and Narasimhan, 1985).

Associated with the pressure drops, the fracture saturations drop abruptly at early times. The matrix changes more slowly from the initial fully saturated condition of $S=1$ to the final ambient saturation of $S=0.8$. These changes are observed in the simulated results. They can also be deduced from the characteristic curves in Figure 4-6. The permeability changes confirm the role reversal between fracture flow at saturated condition and matrix flow after the desaturations. The effective fracture-matrix flow area changes abruptly from $100 \%$ at full saturation to $12 \%$, the contact area fraction. The simulated results suggest that the ambient flow field is through the matrix blocks, the fractures are dry, and the flow lines are distorted in crossing from one matrix block to the next matrix block through fracture contact areas.

In addition to pressure, saturation, permeability, and effective fracture-matrix area changes, we also calculate the Darcy velocity versus time at different locations. With the dependence on both permeability and pressure gradient, the Darcy velocity changes are very sensitive to the details of fracture-flow characteristics. Before the fractures completely desaturate, we have the flows along the fractures change from initially static condition, to large flows associated with fast fracture drainage, to abrupt flow stoppage as the fractures lose the water within the small fracture volume. The fracture-matrix flows are also sensitive to fracture characteristics. Figure 4-10 illustrates the changes in Darcy velocities across the fracture-matrix interfaces at the midpoints of fractures. Before the fractures desaturate, the fluid in the matrix flows toward the fractures to supply the fluid in the fractures which is drained rapidly by the suction. At the saturated. desaturated transition, these fracture-matrix flows change drastically. After the transition, the fluid mainly moves vertically from upper matrix blocks, across horizontal fractures, into lower matrix blocks. The horizontal flow also reverses direction from an initial matrix-to-fracture flow to a small but significant fracture-to-matrix flow. After the transition, the fractures no longer Iransmi fiuiu añu tocome a passive and sma!! fuid storagre source to feed the matrix blocks as they drain. 

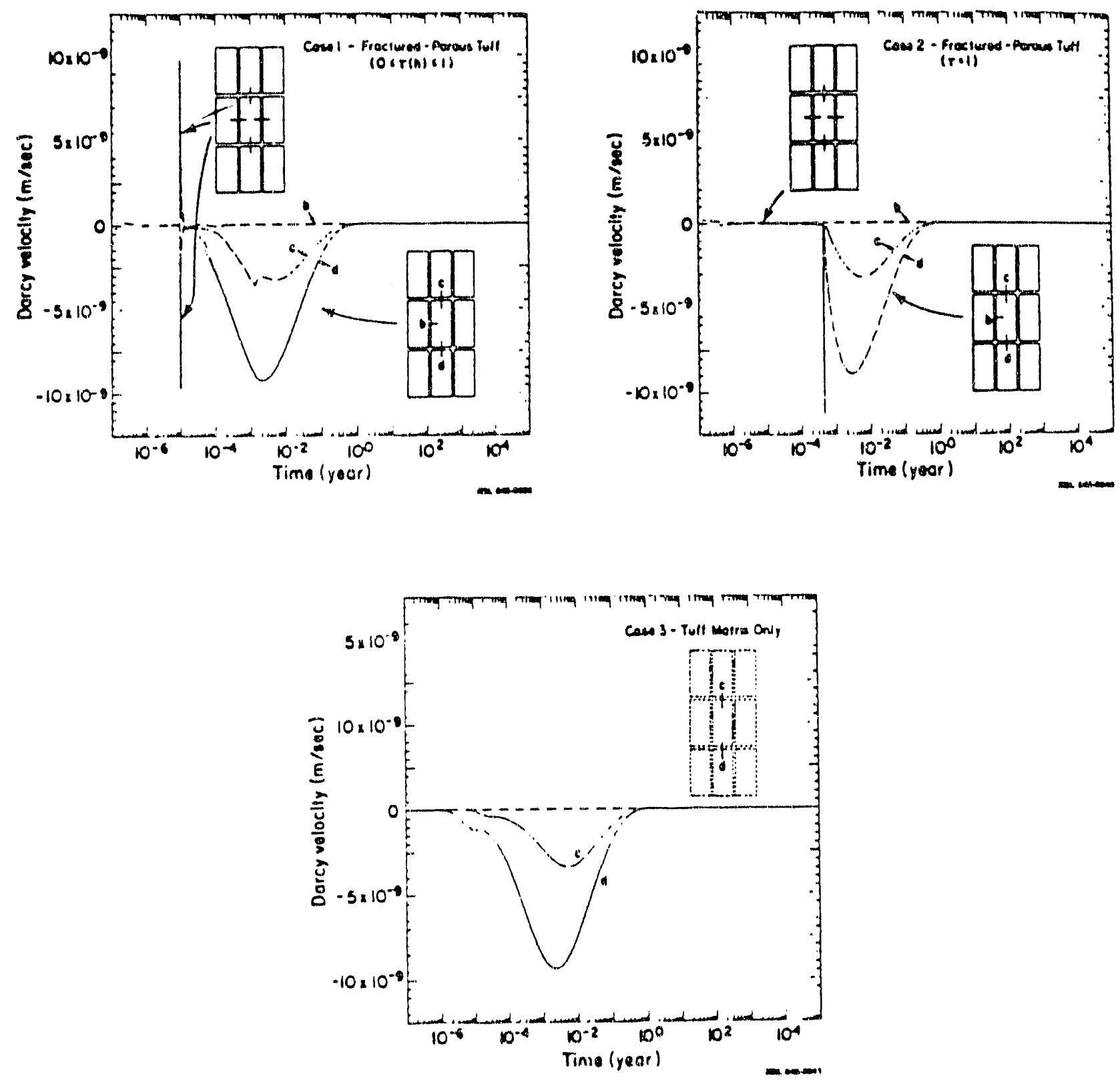

Figure 4-10 Unsaturated drainage in a tuff column: pattems of darcy velocities (sign convention: horizontal fracture - flow into fracture is positive: vertical fracture - flow upward is posilive). 
After the fractures desaturate, the Darcy velocities from one matrix block across the fracture into the next matrix block are essentially the same as those calculated by a matrix-only model, as illustrated in case 3, Figure 4-10 for a tuff matrix column without fractures. The agreement among the velocities in cases 1,2 , and 3 strongly indicates that the fluid flow field of a partially saturated, porous formation can be simulated without taking fractures into account, if the transition region from saturated to unsaturated condition is not of concern.

In quasi steady-state flow, although the dry fractures limit the available areas for flow to cross from one matrix block to another matrix block, the total flux through the fractured column is insensitive to local distortion near the fracture-matrix interfaces. In the 1984 study, we did not simulate the fluid flow field in the scale of individual fracture contacts. The effects of the smallscale heterogeneity are taken into account by the statistical average procedures used in deriving the relative permeabilities and effective fracture-matrix flow areas. Although the total quasisteady flow is not sensitive to the flow path distortion through the fracture-matrix interfaces, the particle velocity field and the solute transpon are expected to be affected by the reduction of fracture-matrix flow areas. We proposed in 1984 to perform explicit simulation of fluid flow field in the scale of individual contact areas to quantify the distortions of particle movements. We have since been diverting our focus to the extension into the study of larger spatial scale for our simulations insiead of amplification of smaller regions around individual contact area at fracturematrix interfaces. We should return to this fundamental process at a small scale to achieve a better understanding of the particle movement through fractured, porous tuff systems.

\subsubsection{A Useful Corollary: The Composite Model}

One of our important findings in 1984 was that the matrix pressure and fracture pressure were approximately the same. This suggests that one may simplify the simulation by considering only one pressure field. The pressure equilibrium, or more generally thermodynamic equilibrium to include ternperature as well as pressure, is one of the essential assumptions of the composite medium-effective continuum models (Peters and Klavetter, 1988; Pruess et al., 1985). With the sarne pressure defined for both the fractures and the porous matrix, one governing equation is sufficient for a fractured, porous matrix system. In the composite medium -effective continuum goveming equation, the equivalent saturation is expressed as a sum of fracture saturation and matrix saturation, weighted by continuum porosities. The equivalent permeability is also expressed similiarly by a weighted sum of fracture permeabilify and matrix permeability. 
There is no conceptual difficulty to accept the assumption that the equivalent saturation is a porosity-weighted sum of fracture saturation and matrix saturation. Saturation is a scalar quantity which is defined as the ratio of saturated volume to pore volume. The permeability, on the other hand, is not a scalar. By summing over fracture permeability and matrix permeability, the fracture flow channel and the matrix flow channel are intrinsically assumed to be parallel in the composite medium-effective continuum models. In view of the popularity and usage in benchmark studies, we have programmed these composite saturation and permeability characteristic functions into the TRUST code. We also used these models later in this project for some of the large-scale simulations. However, there is a need to check the results of simpler models from time to time, with the results generated from detailed discrete fracture models. More important, the assumptions and limits of simpler models must be kept in mind when we use and interpret the results from these models. For example, the composite model is adequate for vertical infiltration studies if we only consider vertical fracture sets so that both fracture flow and matrix flow are vertical. For multi-dimensional simulations with potential lateral flows crossing the fractures, it remains to be determined if composite models are adequate. The development of large scale models to account for both fractures and porous matrix blocks remains to be an important task for the studies of a partially saturated flow field of a fractured, porous system.

\subsection{5: Vertical Infiltrations through Welded-Nonwelded Units}

In 1985, we extended our models from a short column with a few fractures to a tall column over $500 \mathrm{~m}$ in depth to represent the whole unsaturated zone from ground surface to the water table. Yucca Mountain consists of altemating units of welded and nonwelded tuff. The infiltration of water through these alternating layers were studied under steady and transient conditions. We wanted to determine if spatial heterogeneities associated with welded-nonwelded interfaces and temporal variations associated with extreme flooding on the ground surface can induce significant fracture flow in the Topopah Spring welded unit. For performance assessment, the flow velocity field from the repository in the Topopah Spring welded unit to the water table determines the GWTT. The onset of significant fracture flow will shorten the GWTT drastically.

\subsubsection{Fracture and Matrix Data and Model: Observationa! Basis}

To construct models representing the welded and nonwelded units, we analyzed the fracture orientation and spacing data from borehole USW-G4 (Spengler and Chornack, 1984) for all the unsaturated units. For the welded units, Tiva Canyon (TCW) and Topopan Spring (TSw), fractiue 
densities are high and fracture spacings are in the sub-meter range. For the nonwelded units, Paintbrush (PTn), vitric Calico Hill (CHnv), and zeolitic Calico Hill (CHnz), fracture densities are low and fracture spacings are in the meter and tens of meter range. It is generally assumed that flow of water in the nonwelded units is dominated by matrix, and fractures can be ignored. Within fracture planes, we assumed that the contact areas were identical to the areas where mineral coatings occurred in the fractured cores. The fraction of fracture coatings, predominately clay, calcite, and zeolite, increases with depth. This is consistent with the closure of fractures with depth as a result of overburden stress. The equivalent fracture continuum permeabilities for the welded and nonwelded units were based on the estimates of Thordarson (1983) and Sinnock et al. (1984).

For the tuff matrix, we used the characteristic curves of the representative samples chosen by Peters et al. (1984). There is one particular feature in these matrix data which greatly influences our modeling results. For the representative TCw, TSw, and CHnz matrix curves, saturations change slowly with pressure head. Significant desaturation occurs only when the pressure heads are on the order of $-100 \mathrm{~m}$. Saturation of 0.3 or more can be maintained even with pressure heads in the $-1,000 \mathrm{~m}$ range. For the representative PTn and CHnv matrix curves, most of the saturation changes occur over a relatively narrow pressure range around $-100 \mathrm{~m}$. This indicates that the pores in these nonwelded PTn and CHnv representative samples are nearly uniform in size and the welded or zeolitized samples have relatively wider pore-size distributions. We will explain some of our results below with this distinction in matrix characteristics.

We used either a discrete fracture-porous medium model or a composite medium model in the simulations. Before we discuss the detailed models, we will briefly review the results from one-dimensional composite medium simulations. One-dimensional infiltration problems have been extensively studied, especially the steady-state cases. In a one-dimensional column, we can represent the different units with nonuniform grids, as illustrated in Figure 4-11. The thicknesses of the units correspond to the values determined from borehole USW-G4 (Ortiz et al., 1984).

\subsubsection{Estimate of A mbient Infiltration Rate: Observational Constraints}

Although the infiltration rate is a given input parameter and controls the simulation results, it has not been determined by direct measurements, but rather determined inversely by models, ambient saturation, and other characteristic parameters. Especially, the effective matrix conductivity under partially saturated conditions determines the infiltration rate. The saturated 


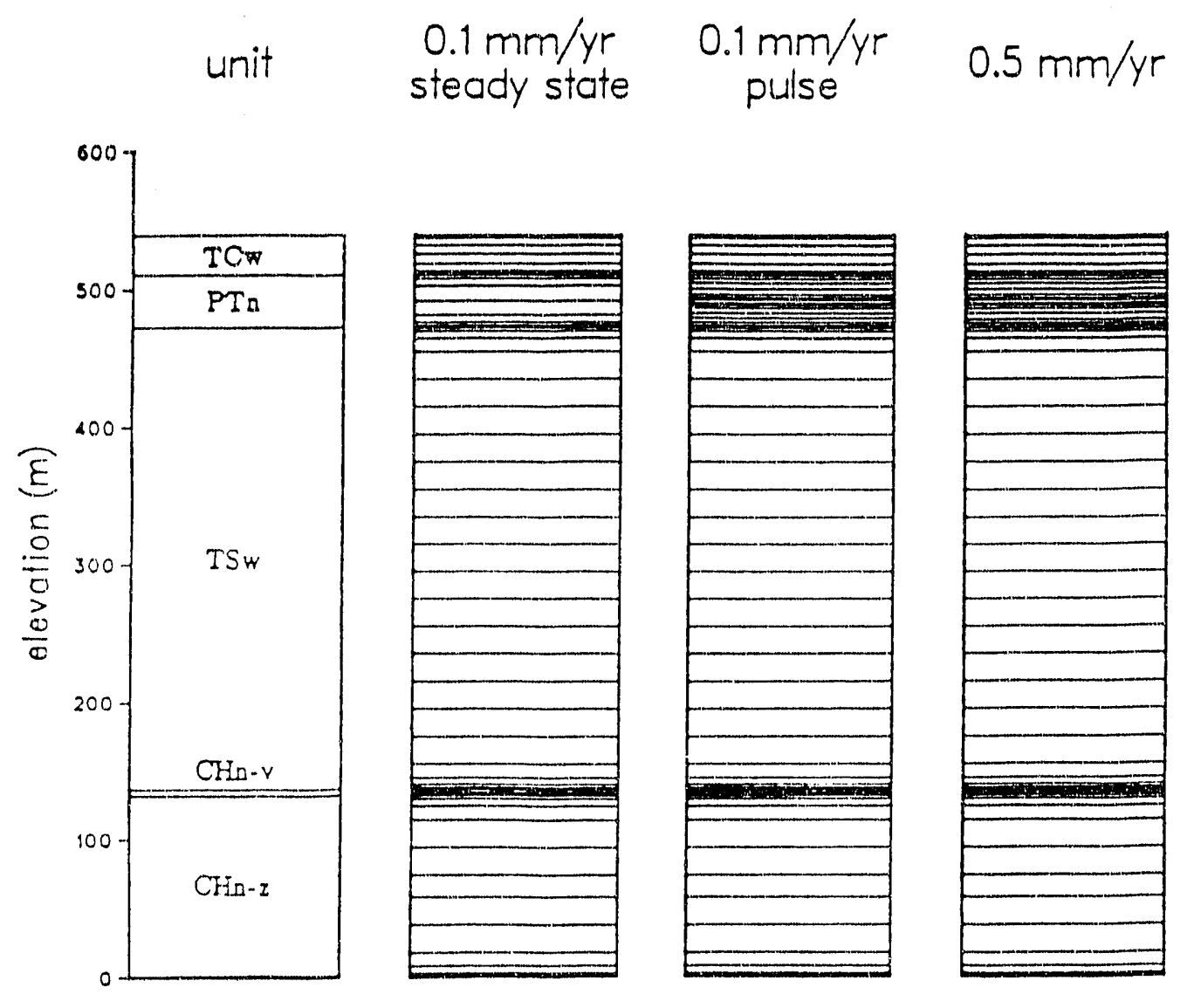

Figure 4-11 Nonuniform grid used for one-dimensional simulations. 
conductivity for the TSw matrix is about $1 \mathrm{~mm} / \mathrm{yr}$ for the representative sample used in our 1985 analyses. Our calculations showed that for the $0.1-\mathrm{mm} / \mathrm{yr}$ case and for the $0.5-\mathrm{mm} / \mathrm{yr}$ case the saturation profiles were uniform and the fluid pressure constant in the upper part of the TSw unit.

In the range of the constant pressure profile, the pressure gradient is, of course, zero, and the hydraulic potential gradient is unity from the gravity component alone. In the range of the unit hydraulic gradient, the partially saturated hydrualic conductivity is equal to the Darcy velocity which is equal to the steady infiltration rate. The solution in the thick TSw unit is essentially determined by demanding the saturation to attain a uniform value at which the infiltration rate exactly matches the partially saturated, effective hydraulic conductivity.

The ambient saturation for TSw was estimated by SNL to be 0.8 in 1983 and later reduced to $0.69 \pm 0.15$ (Sinnock et al., 1984; Reference Information Base (RIB), 1986). With an ambient saturation of 0.8 or lower, the modeling results indicate that one can only allow the system to have $0.1 \mathrm{~mm} / \mathrm{yr}$ or less water flowing through the system. If one increases the infiltration rate over $0.1 \mathrm{~mm} / \mathrm{yr}$, the TSw saturation will be higher than the ambient saturation. The ambient saturation can be easily measured in the field by weighing analyses of core samples. Net infiltration rates, on the other hand, cannot be easily and directly measured. We are not aware of any practical experimental technique to directly measure low infiltration rate under partially saturated conditions. To reproduce the ambient condition for the saturation, we inversely determine the infiltration rate of $0.1 \mathrm{~mm} / \mathrm{yr}$ or less as a reasonable estimate.

Because infiltration simulation is usually cast as a boundary value problem with infiltration rate as an input parameter, there is a tendency for reviewers to ask the simple question as to what the effects of infiltration rate changes are. We can indeed create alarmingly fast fracture flows with infiltration over $1 \mathrm{~mm} / \mathrm{yr}$ which secms to be still a very small infiltration rate. However, the onset of fracture flow is also associated with nearly full saturation in the tuff matrix. At Yucca Mountain, with water table over $500 \mathrm{~m}$ deep, tuff units are in partially saturated states and are not on the verge of becoming fully saturated. If we accept the findings that the tuff units are partially saturated with ambient matrix saturation less than one, and that the matrix characteristic curves can be measured in the laboratories with reasonable accuracy, then we can set a limit on the infiltration rate. This is an important but subtle issue which is frequently raised in analyzing the results 


\subsubsection{Welded-Nonwelded Flow Interaction}

We should point out that the 1985 results have indicated very low saturation values for the nonwelded units. The thick TSw unit at Yucca Mountain is bounded above by a PTn unit and below by a $\mathrm{CHn}$ unit. The $\mathrm{CHn}$ unit is further divided into an upper vitric subunit and a lower zeolitic subunit. The relative thicknesses of $\mathrm{CHnv}$ and $\mathrm{CHnz}$ subunits vary throughout the Yucca Mountain and the CHnv is very thin at well USW-G4 $4.6 \mathrm{~m}$ or $3 \%$ of the total CHn thickness, Ortiz et al., 1985). The saturation values for PTn and for CHnv do not agree with the ambient values of $0.56 \pm 0.17$ for PTn (Sinnock et al, 1984), 0.9 for CHnv, and $0.91 \pm 0.06$ for CHnz. (RIB, 1986). These discrepancies were later reevaluated in 1986 by sensitivity studies for the nonwelded matrix parameters. The PTn and the CHnv units exhibit low saturations because they possess rather uniform pore-size distribution; because of this, the saturation changes very rapidly over a narrow pressure range near $-100 \mathrm{~m}$. For the $0.1 \mathrm{~mm} / \mathrm{yr}$ cases the thick $\mathrm{TSw}$ unit stablizes the pressure profile to $-150 \mathrm{~m}$. The thin nonwelded units are essentially desaturated with saturations near the residual saturation values. The thick TSw unit effectively decouples the upper units from the lower units. The saturation, pressure, and potential profiles in the upper units are insensitive to profiles in the lower units with or without $\mathrm{CHnz}$ below CHnv. The thick TSw unir damps out boundary effects from both the upper and the lower boundaries. This suggests that we may approximately decouple the upper studies of the upper units from those of the lower parts. We used this approximation in some of the pulse infiltration studies.

\subsubsection{Approximate Model for Steady-State Profiles: Elementary Path Integration}

For the transient simulations, the initial conditions used are appropriate steady-state condi. tions. Computationally, the steady-state conditions were obtained by letting the transient process evolve until changes in conditions are negligibly small. If we can estimate the pressure distribution well, we can obtain the steady-state easily. Before we discuss the transient pulse simulations, we will briefly describe a simple and efficient procedure we use to estimate onedimensional steady state pressure distribution without using sophisticated numerical codes. For a vertical one-dimensional column with steady-state infiltration flux q everywhere constant, one can simply integrate explicitly the Darcy's equation,

$$
q=-K(\psi) \frac{d h}{d z}=-K(\psi)\left(\frac{d \psi}{d z}+1\right)
$$

from a boundary with given elevation $z$ and pressure head $\psi$. At the lower water table boundary 
$z=z_{0}=0$, we know the pressure head $\psi=\psi_{0}=0$. With pressure known at that elevation, we can calculate the corresponding conductivity value $\mathrm{K}\left(\psi_{0}\right)$. The pressure at the next node $\Delta z$ above the boundary can be estimated from $\Delta \psi=-(1+q / K) \Delta z$. We can proceed to calculate the conductivity at $\psi_{1}=\psi_{0}+\Delta \psi$, and calculate the pressure head at the next higher node. If $\Delta z$ and $\Delta \psi$ are small enough so that the conductivity value does not change significantly between two neighboring nodes, we can explicitly calculate the pressure profile from the bottom boundary upward node by node. We have incorporated this simple calculation procedure in a mesh generator program as a preprocessor for the TRUST code. We used this procedure in the one-dimensional column simulations and also in two-dimensional multi-column simulations in 1986. Simple procedures such as the one outlined above and the code LLUVIA developed at SNL can be useful and practical tools for sensitivity and statistical analyses.

\subsubsection{Response to Extreme Infiltration Pulses}

The purpose of the transient simulations is to study the responses of the fractured, porous units to extreme infiltration pulses of high rates and short durations. We first focus on the upper layers in Yucca Mountain. We investigate possible scenarios with short-transient infiltration pulses having periodic rates much higher than the saturated matrix conductivity. Figure $4-12$ is a discrete fracture-porous matrix model with the PTn unit sandwiched between two 10-m layers of fractured welded tuff. The discrete model for the welded units contains both vertical fracture grids and horizontal connections from the vertical fractures into the matrix. The PTn unit is treated as a composite medium.

For simulations involving infiltration pulses, the initial conditions correspond to the steady-state solution with a constant infiltration source of $0.1 \mathrm{~mm} / \mathrm{yr}$ on the top TCW node and a sink of $0.1 \mathrm{~mm} / \mathrm{yr}$ on the bottom TSw node. The infiltration pulses are applied to the fracture continuum at the top. The thickness of $\mathrm{TCW}$ unit is greater than $10 \mathrm{~m}$ and varies with the topography at Yucca Mountain. At well USW-G4, TCW is approximately $30 \mathrm{~m}$ thick. By modeling only the lower 10-m section and applying infiltration pulses to the fractures, we assume that the fractures near the surface in the upper part of TCW have apertures larger than the capillary limit and water can penetrate $20 \mathrm{~m}$ of TCW without retention and delay by the tuff matrix. By bypassing the upper portion of TCW, we also neglect the complex near-surface evaporation-transpiration and runoff processes. These processes determine the percentage of precipitation (1) returning upward back to the atmosphere, (2) moving laterally away from the mountain, and (3) infiltrating 


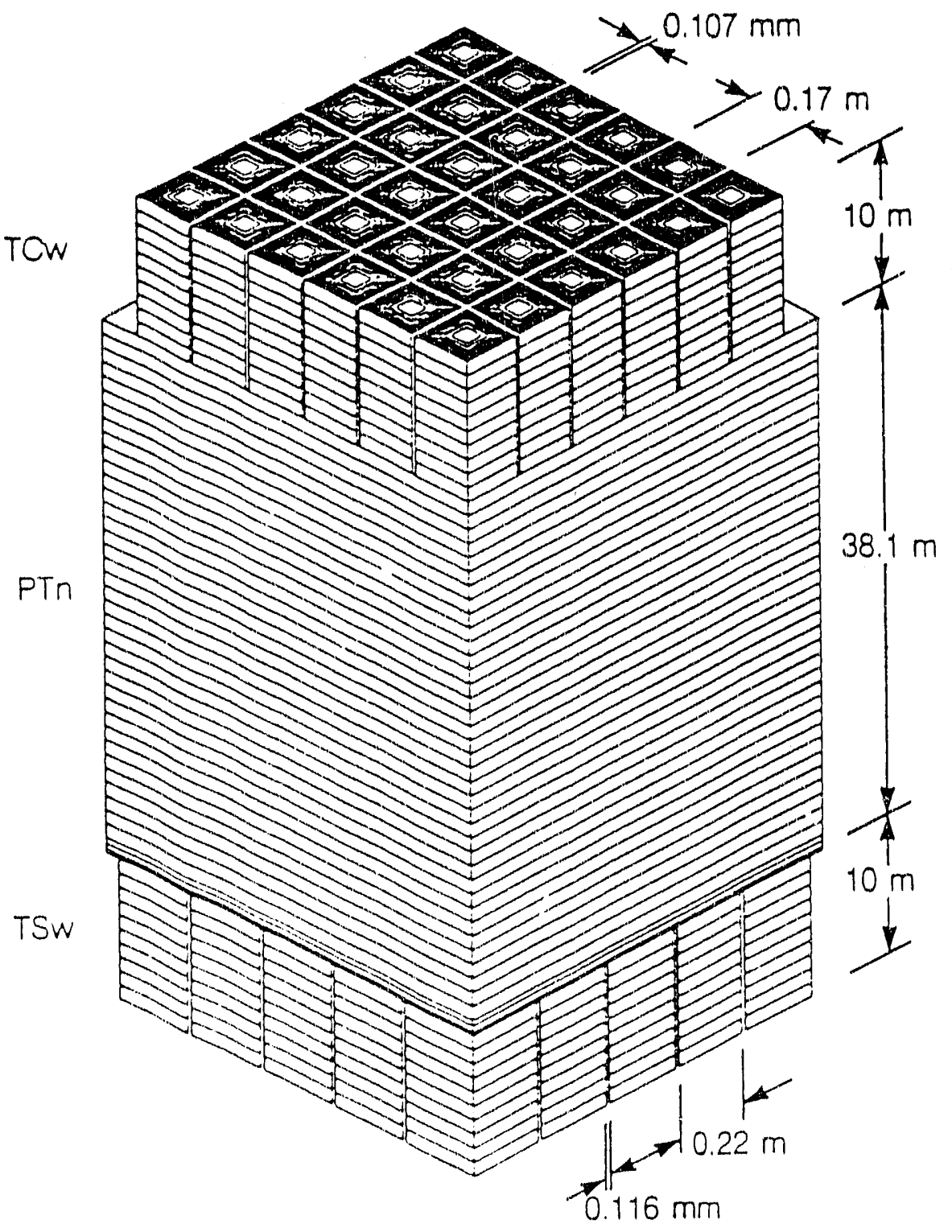

Fïgure 4.12 Three-dimensional mesh used for studying column response to extreme infiltration pulses. 
downward into the ground. We are primarily interested in the effects of net infiltration into the TSw and lower units and the travel time from repository to water table. We assume that the water reaching the top of the lower 10-m section of TCW is the net infiltration.

To systematically study this pulse infiltration problem, first we fix the duration of pulse to $0.2 \mathrm{yr}$, corresponding to an intense rainfall or snow melt period. The pulses are applied periodically. The periods are chosen to be $1 \mathrm{yr}, 5 \mathrm{yr}, 50 \mathrm{yr}, 500 \mathrm{yr}$, and 5,000 yr. For the 1-yr pulse, the intensity of the infiltration is $0.5 \mathrm{~mm} / \mathrm{yr}$, which corresponds to $1 \mathrm{yr} \times 0.1 \mathrm{~mm} / \mathrm{yr}$, divided by 0.2 yr. For the $5,000-y r$ pulse, the intensity of infiltration is $2,500 \mathrm{~mm} / \mathrm{yr}$, which corresponds to $5,000 \mathrm{yr} \times 0.1 \mathrm{~mm} / \mathrm{yr}$, divided by $0.2 \mathrm{yr}$. For the case with 5,000 -yr pulse, all the infiltration in $5,000 \mathrm{yr}$ is applied to the system within $0.2 \mathrm{yr}$. After $0.2 \mathrm{yr}$, the infiltration rate is zero until the start of the next infiltration pulse, $4999.8 \mathrm{yr}$ later. The average infiltration rate over any 5,000-yr period is the sarne as the constant infiltration rate of the initial steady-state condition.

For the mild and frequent pulses ( $1 \mathrm{yr}, 5 \mathrm{yr}$, and $50 \mathrm{yr}$ ), the matrix was found to always remain partially saturated and the fractures essentially dry. The effects of the pulses are completely damped out in TCW. These results are consistent with the findings of Travis et al. (1984) that a slug of water moving through fractures with apertures as small as $0.1 \mathrm{~mm}$ will be unable to penetrate more than a few meters, at most, through fractured welded tuff with matrix saturations as high as 0.9 . Repeated pulsing does not change the capacity of the partially saturated matrix of TCW to absorb all the pulsed water by capillarity. Even for a 50-yr pulse with more than 10 cycles of repeat pulsing, the transient changes are limited within $\mathrm{TCw}$ and the matrix remains partially saturated.

For the $500-\mathrm{yr}$ pulse, the intensity of the infiltration pulse $(250 \mathrm{~mm} / \mathrm{yr}$ for $0.2 \mathrm{yr})$ is high enough to saturate the top $2 \mathrm{~m}$ of $\mathrm{TCw}$. Figure 4-13 illustrates the horizontal saturation profiles within the topmost matrix block of TCW. The pulse is applied to vertical fractures surrounding the four sides of the matrix block.

Al time $t=0$, the first (topmost) discrete fracture continuum node starts to receive all the water at a rate corresponding to $250 \mathrm{~mm} / \mathrm{yr}$ infiltration per unit surface area. Although the water enters the fracture node first, it does not accumulate in the fractures which remain partially saturated. The water is quickly drawn into the matrix by capillarity and distributed nearly evenly throughout the matrix block, which is simulated with 10 nested nodes. The matrix saturations near the matrix-fracture interfaces, $0.085 \mathrm{~m}$ (half the vertical fracture spacing of TCW) away from the matrix block center, are only slightly higher than the matrix saturations in the center of the 


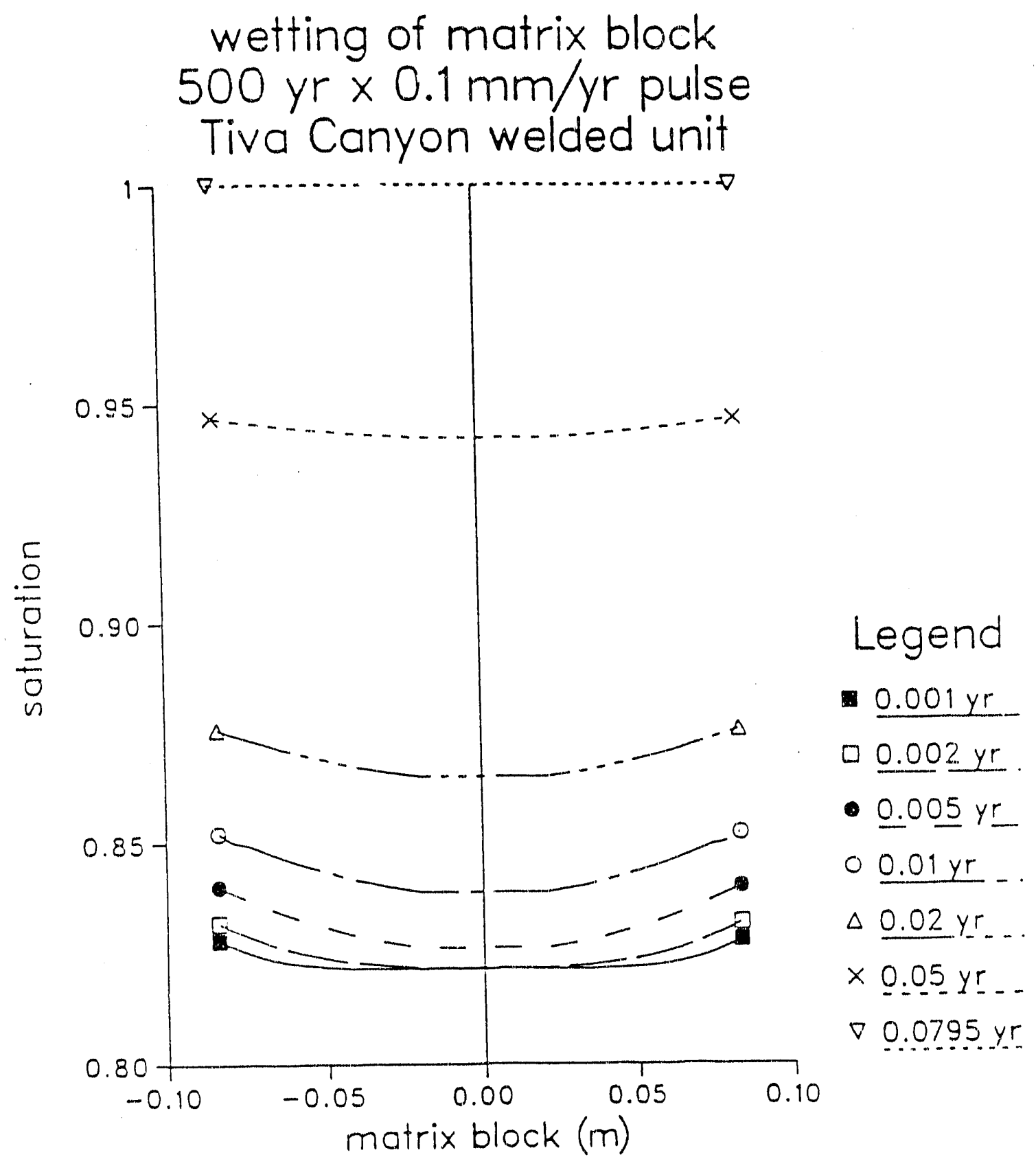

Figure 4-13 Horizontal saturation profile within matrix block of Tiva Canyon welded unit. 
matrix biock. At $t=0.08 \mathrm{yr}$, the matrix block becomes fully saturated $(S=1)$. The total amount of water infiltrated from $t=0$ to $t=0.08 \mathrm{yr}$ is approximately equal to the initial available pore spaces, $\phi(1-S)$, in the topmost matrix block. For TCw with porosity of $\phi=0.1$ and an initial saturation of $S=0.8$, the available pore space in the 1 -m-thick block will be filled in $0.1 \times(1-0.8) \times 1 \mathrm{~m}$ divided by $250 \mathrm{~mm} / \mathrm{yr}$, or $0.08 \mathrm{yr}$. As the matrix approaches full saturation and the negative pressure head approaches zero, the saturation in the fractures increases and water moves down through fractures to the next fracture node. The process of horizontal wetting of the second matrix block follows essentially the same fast-suction, even-wetting behavior of the first matrix block shown in Figure 4-13. Because we can calculate the times required for each block to become fully saturated by the available pore volume and the intensity of pulse without detailed calculations of the horizontal wetting and vertical sharp wetting front movements, the pulse calculations can be greatly simplified as described by Wang and Narasimhan (1986).

The results for the first cycle for a 500-yr pulse showed that changes in saturation, pressure, potential, conductivity, and Darcy velocity occur mainly in the $10-\mathrm{m}$ section in TCW. Full saturation occurs only in the upper part of $\mathrm{TCW}$ during the infiltration period. The pulse effects penetrate slightly into the upper part of PTn but do not reach TSw. Effects of a second cycle are similiar to those of the first cycle. For a 5,000-yr pulse, the $10-\mathrm{m}$ section of TCW is completely saturated during the infiltration period. After infiltration of $2,500 \mathrm{~mm} / \mathrm{yr}$ over $0.2 \mathrm{yr}$, the infiltrating water moves through PTn and reaches TSw in about 1,000 yr. Even for this large pulse, PTn and TSw remain partially saturated. Because the 5,000-yr pulse effects do reach TSw, one may question if the lower boundary used in the simulations was pertiaps too close to the PTn-TSw interface. We did another set of simulations for the 5,000-yr pulse cases using an extended grid.

In the extended grid (see Figure 4-11), we use the comousite-med am model to represent all welded and nonwelded units from the surface to the water table. For the $5,000-\mathrm{yr} \times 0.1-\mathrm{mm} / \mathrm{yr}$ pulse case, an infiltration rate of $2,500 \mathrm{~mm} / \mathrm{yr}$ is appicd for 0.2 yr to TCw below the fully saturated interval which has all its available pore space occupied by infiltrated water. The total amount of water applied to the system in $0.2 \mathrm{yr}$ corresponds to all the infiltration in $5,000 \mathrm{yr}$. The results of the transient profiles of saturation, pressure head, potential, conductivity, and Darcy velocity for the first $5,000 \mathrm{yr}$ showed that the zone of full saturation does not penetrate the PTn. Similiar results are obtained for additional cycles of pulse infiltration. The damping of the pulse and the penetration of the transient effects for this composite-medium, all-unit simulation agrees with the earlier discretc-fracture, upper-unit results with finer mo shes. The effects of the pulsing 
reach the top of TSw in about 1,000 yr and penetrate only the upper part of TSw.

Most of the damping of the pulse occurs in PTn. A large 5,000-yr pulse can saturate the matrix, initiate fracture flow, and generate complex flow field in $\mathrm{TCw}$. When the transient effects move into PTn, its large pore volume and highly nonlinear unsaturated characteristics can effectively accommodate and absorb the highly transient and intense water pulses. Only mild perturbations reach TSw. Figure 4-14 illustrates the propagation and damping of the pulse in PTn. The large mass flux passing through the top TCW-PTn interface at $100 \mathrm{yr}$ is damped out as it moves down PTn. The mass flux crossing the lower PTn-TSw interface into TSw is much smaller in magnitude and later in time.

\subsubsection{Estimate of Lateral Flows}

In this extended grid, composite-medium column simulation, we also use a fixed-gradient approximation to estimaie the potential lateral flow which may divert a fraction of infiltration from the verical direction along the column. The results in Figure 4-14 includes the effects of lateral leakage from anisotropic PTn. The usefulness and limitation of the fixed gradient approximation are discussed by Wang and Narasimhan (1986). Because we went beyond the single vertical column to more accurately estimate the effect of lateral flow in 1986, we will omit further discusion on the fixed gradient approximation.

\subsubsection{Ambient Infiltration: An Estimate}

Our 1985 results support a conclusion that water flow occurs mainly through the tuff matrix if the average riet infiltration rate is less than the saturated conductivity of the matrix. Spatial heterngeneities associated with welded nonwelded interfaces and temporal variations associated with large infiltration pulses do not change the flow field at TSw significantiy. The saturated conductivity of the welded tuff matrix is very small from laboratory measurements. With small salurated conductivity in the range of $1 \mathrm{~mm} / \mathrm{y}$, the net infiltration rate is estimated to be $0.1 \mathrm{~mm} / \mathrm{s}$ or less so that the simulated saturations agree with the ambient values of 0.6 to 0.8 for the welded units. If the onset of fracture flows could be demonstrated $w$ be unlikely under ambient conditions and under extreme flooding events and the dominant flow direction is vertical, performance assessment could be easily and convincingly carried out for the partially saturated, fractured tufí al Yucca Mountain 


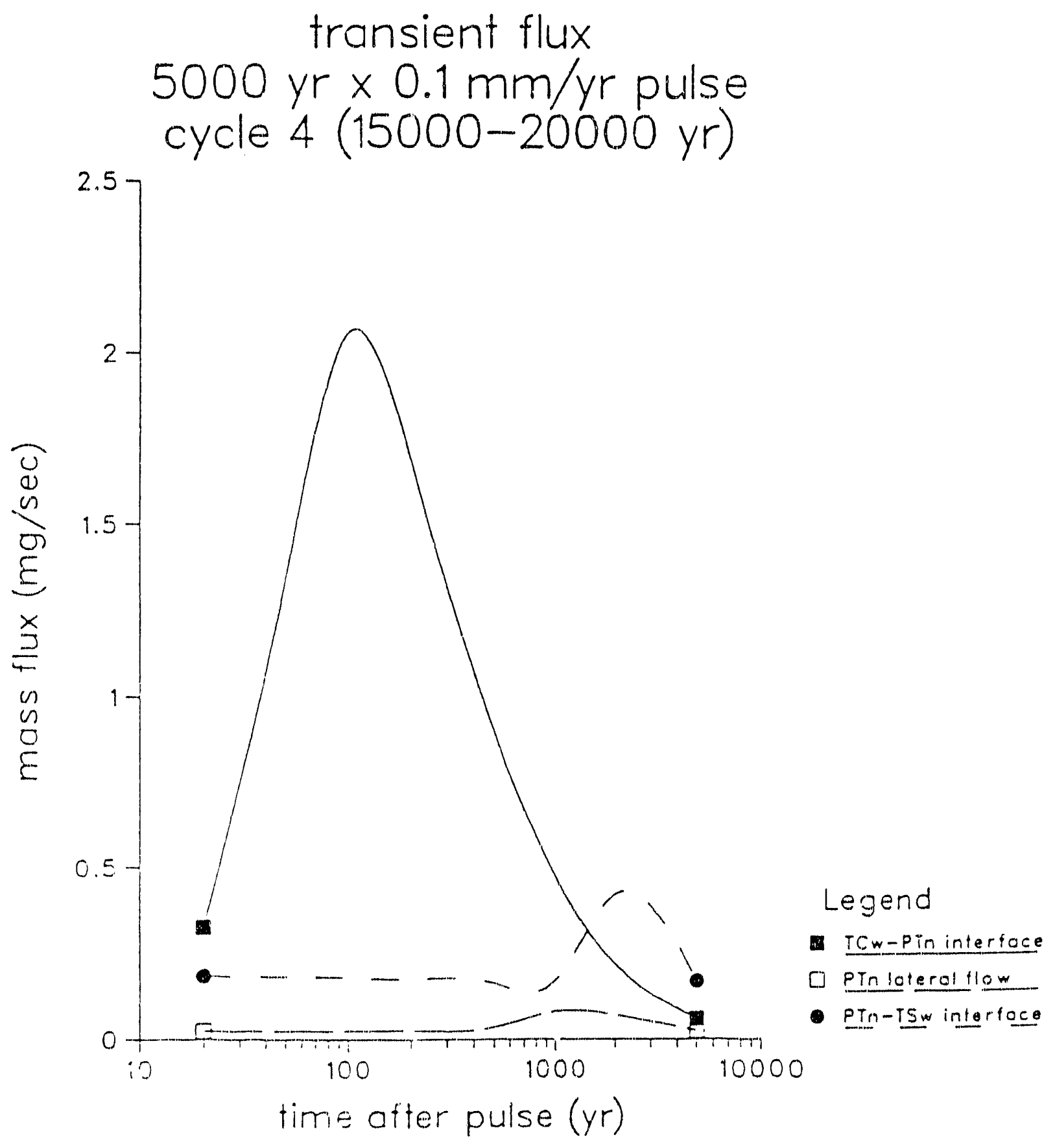

Figure 4-14 Response to 5,000-yr infiltration pulse: damping of fluxes within the Paintbrush unit. 


\subsection{6: Distribution of Fluid Near a Fault Zone: Possibility of Lateral Flow}

In 1986, we extended our models from a single vertical column to a two-dimensional eastwest cross section bounded by vertical fault. Yucca Mountain is approximately $2,000 \mathrm{~m}$ wide. A minor fault, the Ghost Dance Fault (GDF), inside the proposed repository area, will be a major hydrological concern if the fault flow is an important transport mechanism. Without experimental data on the unsaturated characteristics of the fault zone material, we focused our attention in 1986 on the distribution and movement of water in the unsaturated formation near GDF and assessed the potential for water to overcome capillary forces and exit laterally through the formation-fault interface. If water can be diverted away from dispersed vertical flow through the unsaturated formation to concentrated flow through the localized fault zone, the fault zone would likely constitute the fastest flow path from the repository to the water table. On the other hand, the absence of significant fault flow and the dominance of matrix flow will result in long GWTT through the unsaturated tuff units.

\subsubsection{Unsaturated Fault}

The distribution of fluid flow in the vadose zone is governed by the balance between the capillary forces and gravity. The extemal gravitational force moves the water downward, and the internal capillary forces hold the water in place within the pores. The magnitude of capillary force is inversely proportional to pore size and the heterogeneity of rock pore structure controls distribution and movement of water through partially saturated formations. Within the meter scale, we have the heterogeneity with tuff matrix blocks separated by discrete fractures. The large fracture openings cannot maintain the strong capillary forces required to hold water within discrete fractures under ambient partially saturated conditions. In the much larger scale covering the mountain width, we have the faults as the heterogeneous feature.

In modcling the hydrology of Yucca Mountain, it is important to assess the impacts of faults that traverse the tuff formation. If faults are barriers to flows, faults will partition the system into separate hydrologic blocks, and we can focus on the study of flows through the interior of blocks. If faults are the main conduits for fluid, then we should focus on the study of fault flow's. In saturated syems, we have a clear distinction between these two possibilities. A closed fault is treated as a no-flow boundary, and an open fault as a constant potential boundary if it is connected to a constant-head reservoir. In the latter case, faults may inelp transpon water from a far-removed source or may he!p remove water to a deeper sink. 
In partially saturated systems, we may not have such a clear distinction between an open and a closed fault. Three possibilities can be considered:

- If a fault zone is made up of broken up mass because of brecciation, it might have very coarse openings that can be easily drained. If the openings are dry, there is no fluid to be transported. On the other hand, a dry opening is not a barrier to fluid flow. If water enters the opening, it will have high permeability and offer low resistence for fluid to slide down the walls of large openings (noncapillary flow). Under partially saturated conditions, an "open" fault becomes a passive boundary which can transport water if water enters it, but it cannot keep water because there is only very weak capillary force to hold water in large openings.

- If a fault is sealed with gouge material and fine-grained materials, it can retain water and may even attract water with the strong capillary force associated with smal! openings. On the other hand, small openings have low permeability and cannot be good conduits for transporting fluid. Under partially saturated conditions, a "closed" fault becomes a relatively wet zone that passively retains water in nearly static conditions.

- There is a third, remote, possibility that the fault may have a unique combination of saturated and unsaturated characteristics. If the fault material can maintain both strong capillary attractive force and large permeability, then the fault can pull water away from the formation and transport it quickly away from the system. We cannot rule out the existence of such a unique combination wherein fault flow would control the partially saturated hydrology.

\subsubsection{How to Model the Fault: The Seepage Face Idealization}

There are no data for the faults to clearly distinguish whether faults in tuff formations have open, closed, or exotic characteristics. In the 1986 study, we did not use material properties to represent the fault. We treat the fault-formation interface as a seepage boundary. It is well known from hillside studies that water will seep out of the formations if the potential at the open face is lower than the potential inside the hill. We assume that the fault is so open that capillary force is zero and the face of the fault behaves like a hillside open surface exposed to atmospheric pressure. We focus on the interior of the formation to determine whether water can overcome capillary forces holding water inside the tuff formation. If water does not exit through the faultformation boundary, the seepage boundary is essentially a closed boundary. If water remains inside the formations, distribution of fluid flow will be independent of whether the fault is open or closed. The only possibility we do not model is the third possibility, wherein the fault acts as a strong capillary suction sink as well as a high-permeability conduit under parti ly saturated conditions. 
Within the tuff formation, the lateral component of the gravity gradient caused by the tilting of the units has been proposed as a mechanism to divert water laterally (Montazer and Wilson, 1984; Rulon et a.r. 5986 ). The stratigraphic units at Yucca Mountain generally tilt eastward at $5^{\circ}$ to $7^{\circ}$ (Scott and Bork, 1984). For an average $6^{\circ}$ tilting, the tangent of $6^{\circ}$ is 0.1 and the external driving force along a lateral direction is $10 \%$ of gravity. The partially saturated flow movement is determined by the balance of external force with internal capillary forces. We expect that a weak extemal force associated with the lateral component of gravity will demand a mild readjustment in lateral variation in saturation, capillary pressure head, and potential to allow the capillary force balances out the external force.

\subsubsection{Stratigraphy, Fracture, Matrix Data: An Update}

Before we present our results from the two-dimensional cross section models, we briefly discuss several preliminary steps and the systematic approaches we adapt in this study. In 1986, a Reference Information Base (RIB) was developed at SNL. Thomas Lin of SNL provided us with digitized data for section A-A' from RIB. Section A-A', illustrated in Figure 4-15, is one of the sections used by Sinnock et al. (1984) in a multi-column unit-gradient vertical-flow stochastic simulation to study the GWTT distributions. To focus on the impact of the GDF on the twodimensional flow behavior in section A-A', we first repeat the single column simulations with thicknesses of the units corresponding to digitized values at GDF instead of borehole USW-G4 in our carlier studies. We also update fracture and matrix data according to RIB and other new SNL reports. For examples, the average fracture orientation and frequency data from five boreholes (Bauer. 1986) was used instead of the early analyses with USW-G4 data alone. The main difference between the updated results and early analyses is that the iorizontal fractures are relatively fewer than the vertical fractures, especially for the welded units. For the matrix, the RIB saturated conductivity values for TSw and $\mathrm{CHn}$ matrix are lower than corresponding values in earlier references. With lower saturated conductivity, the calculated saturation in TSW is approximately $5 \%$ higher than the 1985 result for the same infiltration of $0.1 \mathrm{~mm} / \mathrm{yr}$.

In the RIB (1986), the characteristic parameters are not tabulated. We still used the data of Peters et al. (1984) for these parameters. Until 1986, we had been focusing attention on understanding fraclure characteristics and relied on SNL to provide matrix characteristics. In 1986, we also started to analyze available matrix data. The comprehensive repon by Peters et al. (1984) contians much data and analyses of laboratory measurements of small intact as well as fractured tuff core samples. In our 1986 simulations, the matrix characteristic parameters for TSW1, TSw2, 


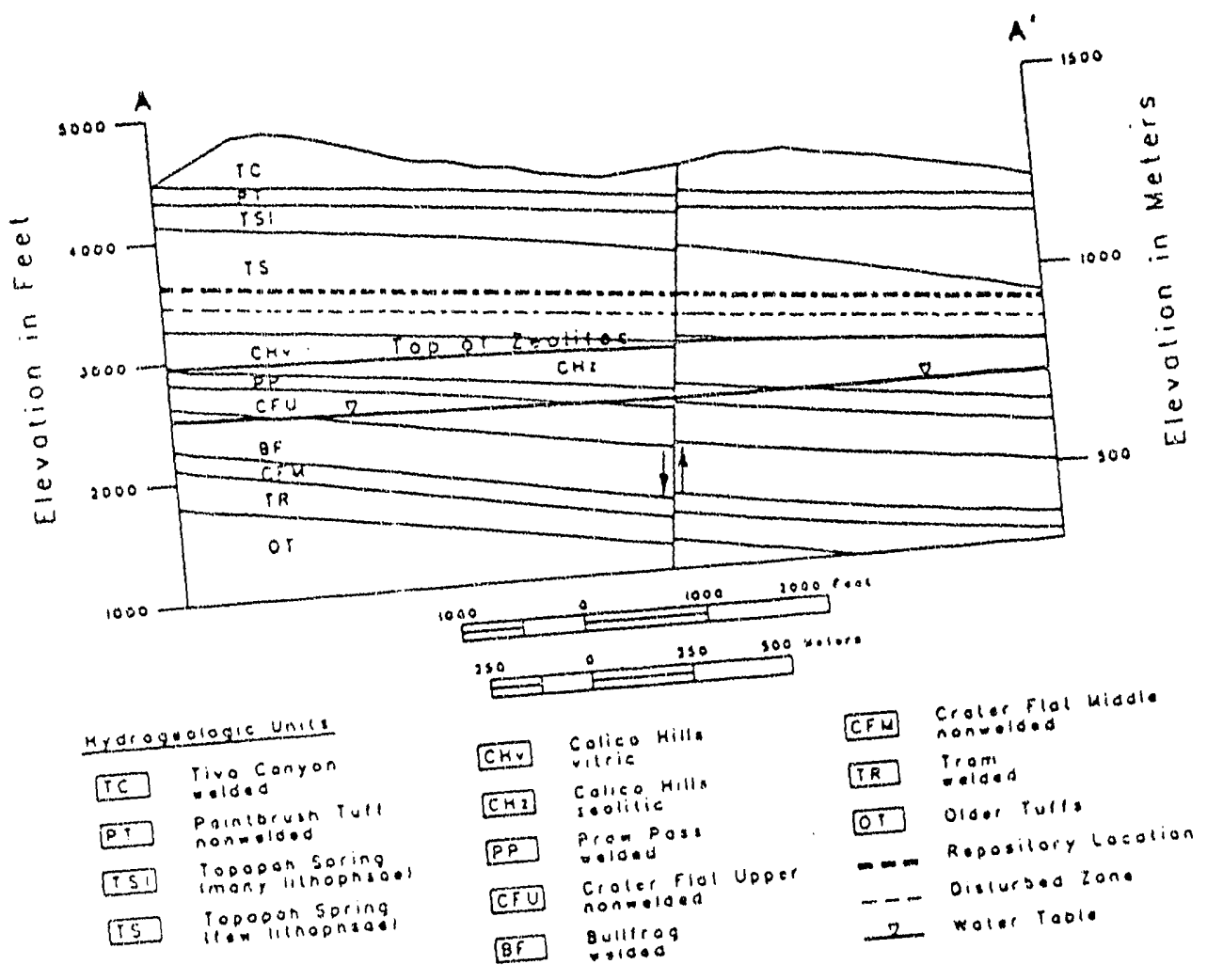

Figure 4-15 Idealized cross section of Yucca Mountain. 
CHnz, and PPw units correspond to the representative samples chosen by Peters et al. (1984). For PTn and CHnv, we used two other samples with wider pore size distributions which were also from Peters et al. (1984). These two nonwelded units may have multi-modal pore structure as shown in later detailed PTn study by Peters et al. (1987). Recent study by Paul Kaplan indicates that the CHnv unit is very heterogeneous with many subunits of different characteristics.

\subsubsection{The Ghost Dance Fault: A Column Profile}

With the use of characteristic curves representing wider pore size distributions in our 1986 study, we were able to yield simulated saturation values closer to measured ambient saturations for the nonwelded units as shown in the GDF column results. The evolution of the 1986 results from the 1985 results represents our conscious efforts to update our data and improve our model predictions. All our model parameters are determined from available data. In 1985 and earlier, we had the wrong impression that the nonwelded units are porous and homogeneous. The predic- ns of low saturations in nonwelded units using matrix characteristic curves with narrow pore size distribution bothered us. In 1986, new studies and observations suggested that the nonwelded units are heterogeneous, and the updated predictions reduced the discrepancies between model predictions and experiment measurements. We expect that the continuing process of cross checking between models and measurements will validate the models for performance assessment.

Before implementing two-dimensional grids, we conducted tests with several onedimensional cases to study the effects of mesh refinement and spatial averaging of permeability on the solution. Our calculations showed that we did not introduce any serious errors because of our choice of the mesh refinement or the method of spatially averaging permeability.

\subsubsection{Multi-Column Models}

To focus on the effects of eastem tilting of the units and the presence of GDF on the twodimensional flow behavior, we modeled the region west of GDF, treating GDF as a scepage boundary. The location of the westem boundary was treated as variable ranging from $190 \mathrm{~m}$ to $648 \mathrm{~m}$. Five meshes were used in the two-dimensional studies to evaluate the effects of mesh refinement and the effects of system boundaries. Our results showed that numerical uncertainties associated with lateral mesh refinements were small. With mild variations in saturation, pressure, and potential in the lateral direction, coarse gridding can be used to generate acceptable results. 


\subsubsection{Fluid Redistribution within the Tuff Formation}

The two-dimensional simulations showed that the eastern tilting of the units results in slightly higher matrix saturations in the eastern side of the cross section. Consequently, pressure heads along eastern columns closer to the seepage boundary are slightly higher than pressure heads along western columns. Pressure head values remaiv negative and deviations of twodimensional results from one-dimensional one-column results were found to be small. Deviations of two-dimensional potential distributions from one-dimensional potential distributions were found to be even smaller. From matrix saturation, pressure head, and potential results of the two-dimensional multi-column model, tilting of the units changed vertical distributions very little.

The matrix saturation and pressure head values of the nodes in the middle of TSw2 unit were found to be higher in the east than in the west. From saturation differences between the eastemmost and westernmost columns, we calculated that the rate of linear saturation change varied from 5 to $17 \% / \mathrm{km}$, depending on the number of columns in the model; the smaller the number of columns, the larger the change. From results of the 9-column, 648-m-section model, we can also estimate wider section upper bounds for the change rate of other variables: pressure head: $60 \mathrm{~m} / \mathrm{km}$; potential, $44 \mathrm{~m} / \mathrm{km}$; vertical Darcy velocity, $0.085 \mathrm{~mm} / \mathrm{yr} / \mathrm{km}$; and vertical matrix pore velocity, $0.79 \mathrm{~mm} / \mathrm{yr} / \mathrm{km}$.

\subsubsection{Vertical and Lateral Flow}

Calculations using the 9 -column, $645-\mathrm{m}$ model showed that vertical Darcy velocity along columns closest to the GDF boundary is approximately $25 \%$ higher than the $0.1-\mathrm{mm} / \mathrm{yr}$ infiltration rate; flow in column 5 , in the middle, is approximately $0.1 \mathrm{~mm} / \mathrm{yr}$, and flow in column 9, away from the GDF boundary, is approximately $25 \%$ lower than $0.1 \mathrm{~mm} / \mathrm{yr}$. From the vertical matrix velocity, we can calculate the GWTT from the repository to the water table. For the 9column, 648-m model, the GWTT along the eastenumost column is approximately $50 \%$ shorter than the travel time along the westernmost column. If the width of the section is doubled, we expect the GWTT to vary in the range of a factor of 2 from different points in the repository. The fluid flow redistribution effects induced by the tilting units are unlikely to introduce orders-ofmagnifude changes in GWTT for the $0.1-\mathrm{mm} / \mathrm{yr}$ infiltration cases.

In slanted meshes used in this study, lateral fluxes between two ncighboring columns are evaluated from both pressure and elevation differences of neighboring nodes. Because elevation 
differences are included in flux calculations, a lateral velocity is not a horizontal velocity. Lateral velocities measure flux magnitudes along layers parallel to tilted interfaces. A careful evaluation of the lateral fluxes between the columns showed that lateral Darcy velocities are smaller than vertical Darcy velocities.

When it does occur, lateral flow occurs mainly within the PTn unit. Lateral flow is the mechanism to redistribute vertical infiltration by channeling water from the west to the east, so that vertical Darcy flux in the east is higher than in the west, and saturation is higher in the east than in the west.

Magnitudes of lateral flow were found to be higher in the middle of the cross section than near the side boundaries. In other words, water does not exit through the fault-formation boundary. The lateral gravity gradient associated with tilting of interfaces cannot overcome capillary forces that hold water in partially saturated formations.

\subsubsection{Onset of Seepage Flow}

For the $0.1-\mathrm{mm} / \mathrm{yr}$ cases, representing the ambient saturation conditions, no seepage flow occurs through the formation-fault boundary. The onset of seepage flows is simulated in the 0.5$\mathrm{mm} / \mathrm{yr}$ infiltration cases. With $0.5-\mathrm{mm} / \mathrm{yr}$ infiltration, the tuff units are nearly saturatei and seepage flow was found to occur only at the CHnv-CHnz interface for a 5-column, 343-m section model with $0.5-\mathrm{mm} / \mathrm{yr}$ infiltration. In terms of inass fluxes, we have approximately $8 \%$ lateral diversion; i.e., $8 \%$ of infiltration on the ground surface exits at the CHnv-Chmz interface out of the two-dimensional section, and the remaining $92 \%$ moves down to the water table. For this casc, the amount of water flux driven by the lateral gravity gradient parallel to tilted interfaces is larger than the matrix saturated conductivity, and fracture flows arc initiated at the seepage exit point. With the material properties used in the 1986 study, we observed seepage flow only in the CHnv-CHnz interface for the $0.5-\mathrm{mm} / \mathrm{yr}$ cases. If we use different characteristic curves for the units. we may also generate results with seepage flow in other units if the units have pressure values nearly zero and redistribution effects are strong enough to increase pressure values to nonnegative ranges. We should reemphasize that saturation and pressure distributions with 0.5 mi 'yr infiltration do not represent ambient partially saturated conditions at Yucca Mountain. Therefore, predictions of the existence of seepage flows in the $0.5-\mathrm{mm} / \mathrm{yr}$ cases were mainly modeling excrcises. 


\subsubsection{Ambient Flow Field}

Based on results with two-dimensional cross section models for alternating layers tilted toward the seepage boundary on the east, we may conclude the following for $0.1-\mathrm{mm} / \mathrm{yr}$ infiltration:

- Eastern tilting of units contributes to redistribution of flow, resulting in higher saturations and higher vertical velocities in the eastern side of a block bounded by faults. This redistribution effect is approximately proportional to cross-section width.

- Nonwelded units are major channels in redistributing vertical infiltration. Magnitudes of lateral flow are higher in the middle of the cross section than near side boundaries.

- Tilting itself is not a strong enough mechanism to induce drastic changes in saturation, pressure, and potential distributions to drive flows into fault zones.

The mild lateral variations in saturation, pressure, and potential indicate that onedimensional results are fairly good estimates of ambient conditions. The lateral tilting mechanism is a second-order peiturbation that does not globally change the downward, gravity-driven flow patterns. So long as fluid flow remains inside partially saturated units, redistribution effects caused by tilting will not introduce order-of-magnitude differences in fluid transport predictions. If the system is on the verge of making a phase transition from partially saturated to fully saturated condition, as in the case of $0.5-\mathrm{mm} / \mathrm{yr}$ infiltration, the lateral tilting mechanism can perturb the system and change a fraction of fluid flow from dispersed slow flows percolating through the formations to concentrated fast flows rushing down the faults. If fault flow is important, the carly arrival portion of the GW'TT distributions may be controlled by the fault characteristics. For ambient conditions with large negative pressure values in all partially saturated units, it is unlikely that the lateral tilting mechanism can perturb the pressure and saturation field significantly enough to overcome negative capillary forces holding water in formations and change GWTT distributions drastically.

\subsection{7: Statistical Analyses of Tuff and Soil Data}

In 1987, we systematically analyzed the hydrological parameters of tuff matrix and fractures. Because many hydrological parameters are cietermined from microscepic pore structures, together with fluid properties and rock textures, correlations among different parameters may manifest themselves in the measured values. To elucidate the parameter correlations, we compile and compare saturated and unsaturated parameters of tuff matrix with watershed soil data. There 
are two reasons for us to analyze the tuff matrix and soil data in detail. We are concerned with the proper range of the fault and fracture parameters which are difficult to measure but may control the system response in the fractured tuff formations. Also the determination of GWTT distribution requires statistical quantification of model parameters. We conclude this section on statistical analysis with a discussion of the application of fractal theory to the analysis of roughwalled fractures and the relationships between fractal theory and geostatistics.

\subsubsection{Parameter Distributions}

Because many unsaturated flow models were developed in soil studies, it is important to analyze the similiarity and difference between soil and tuff rock characteristics. Some of the soil data have also been used to represent fractures and faults in tuff formations. We compared parameter distributions of tuff matrix data with watershed soil data. Specifically, the tuff matrix data are from the SNL studies by Peters et al. (1984) and Klavetter and Peters (1987), fitted with the van Genuchten (1980) model. The watershed data are from the USDA study by Holtan et al. (1968), fitted by Panian (1987) of Desert Research Institute, also with the van Genuchten model.

In the van Genuchten model, the dependence of effective saturation $S_{e}$ on suction pressure head $h$ is described by the equation,

$$
S_{e}(h)=\left[1+(|\alpha h|)^{n}\right]^{-1+\frac{1}{n}}
$$

Figure 4-16 compares the van Genuchten curves with the popular Brooks and Corey (1966) model,

$$
\begin{gathered}
S_{e}(h)=1 \text { if }|\alpha h|<1 \\
S_{e}(h)=|\alpha h|^{-\lambda} \text { if }|\alpha h| \geq 1 .
\end{gathered}
$$

In the range of large suction, the van Genuchten equation is asymptotically equivalent to the Brooks and Corey power function with the pore-size distribution index $\lambda=n-1$. The inverse of the parameter $\alpha,\left|h_{a e}\right|=\alpha^{-1}$, is frequently referred to as the air entry value or bubbling pressure in the soil literature. This is one interpretation for the parameter $\alpha$ or $h_{a e}$. When suction pressure head $h$ overcomes the air entry pressure $h_{\mathrm{ae}}\left(|\mathrm{h}| \geq\left|\mathrm{h}_{\mathrm{ae}}\right|\right)$, air enters the pores and water flows out of the medium to initiate desaturation. This interpretation is based on the cutoff separating the $S_{\mathrm{t}}=1$ saturated region from the $S_{\mathrm{e}}=|\alpha h|^{-\lambda}$ desaturated region in the Brooks and Corcy model. Another interpretation for $\alpha$ is to treat it as a scaling parameter. The saturation depends 


\section{Characteristic Curves}

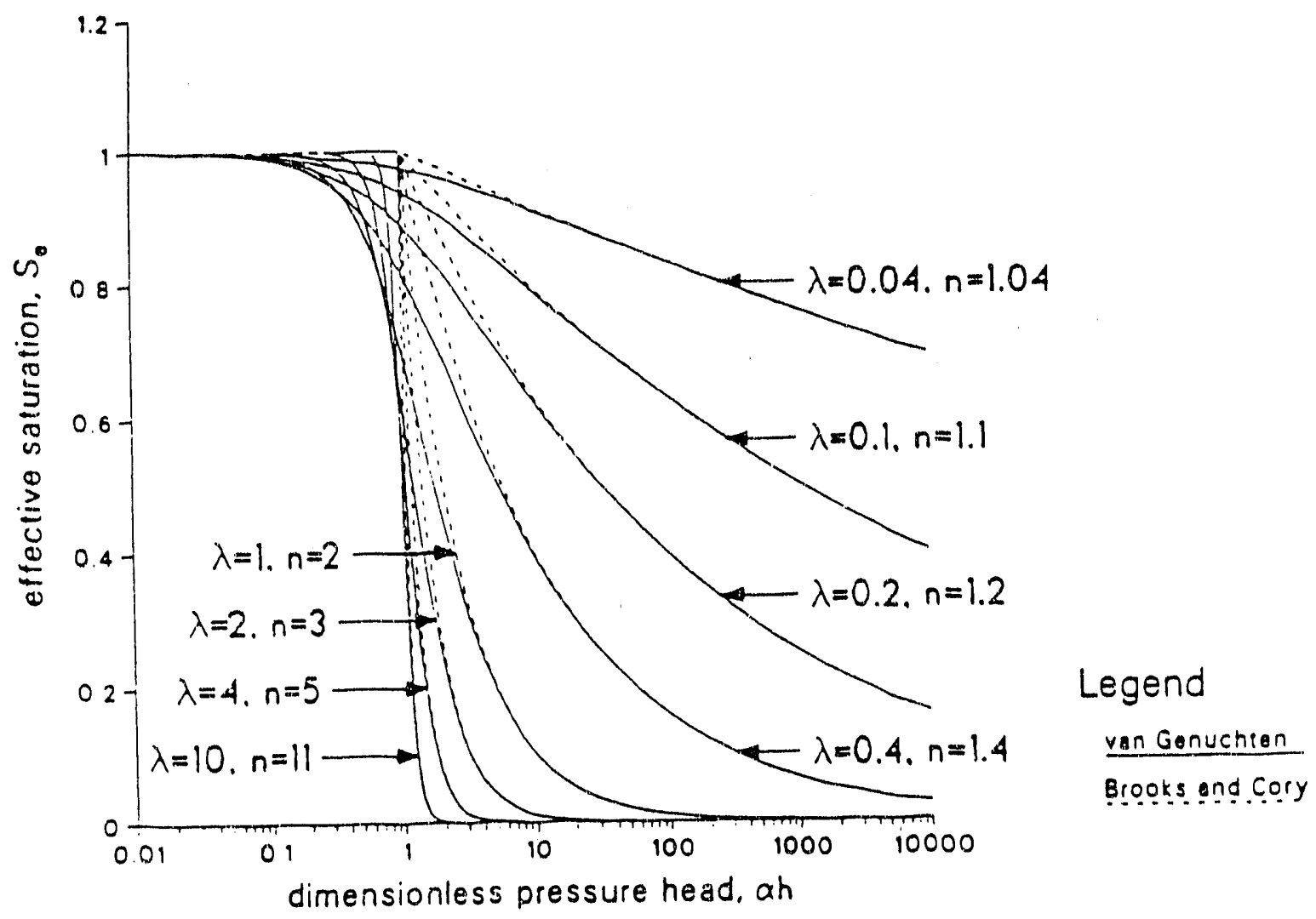

Figure 4-16 Comparison of parameters of the van Genuchten model and the Brooks-Corey model. 
on $\alpha$ h together and not on $\alpha$ and h separately. Characteristic curves of soils with different $\alpha$, but same $\lambda$, can be scaled into one curve. Therefore, we refer to $\alpha$ as the air entry scaling factor. If flow channels are represented by capillary tubes, we can define air entry radius by the capillary equation $r_{a c}=-2 \sigma / \rho g h_{a c}$. With surface tension $\sigma$, water density $\rho$, and gravitational acceleration $g$ as constants, $r_{\mathrm{ae}}$ is proportional to $\alpha$. We can treat the air entry radius $\mathrm{r}_{\mathrm{ac}}$ as a parameter equivalent to the air entry scaling factor $\alpha$.

We have analyzed the pore-size distribution index and the air entry scaling factor, together with saturated permeability, porosity, and residual saturation. Detailed analyses of the distributions and the correlations of soil and tuff parameters are in the report by Wang and Narasimhan (1988). In this review, we focus on two parameters: the saturated permeability and the unsaturated air entry scaling factor. Figure 4-17 compares the distributions of $\log$ air entry scaling factor of watershed soils with tuff matrix. The important cbservation is that the average air entry scaling factor for watershed soils is two to three orders of magnitude larger than both the welded tuff and nonwelded tuff. Because air entry radius is proportional to air entry scaling factor, the order of magnitude differences simply indicate that the soils have much larger pores that can be easily desaturated and tuff matrix have smaller pores that are more difficult to desaturate. Figure 4.18 shows that the average saturated permeability for watershed soils is five to seven orders of magnitude larger than those for welded/zeolitized tuffs.

\subsubsection{Parameter Correlations}

The correlation between saturated permeability and air entry radius based on mean values of these parameters for different soil texture categories and different tuff stratigraphic units are plotted in Figure 4-19. If a porous medium is represented by uniform circular tubes with radius $r_{a e}$, the maximum permeability of this idealized air entry tube model is $k_{a e}=r_{a e}^{2} / 8$. Figure 4-19 shows that the correlation between saturated permeability and air entry radius follows approximately the square functional dependence when soil data are compared with welded/zeolitized tuff matrix data. Wang and Narasimhan (1988) discuss other data sets in the literature on this and other parameter correlations.

In the literature, the correlation between permeability and porosity has been extensively studied. If the saturated permeability does correlate with other hydrological parameters, it probably correlates better with air entry radius than with porosity. Physically, this correlation makes more sense with air entry radius than with porosity. The saturated permeability measures the flow 


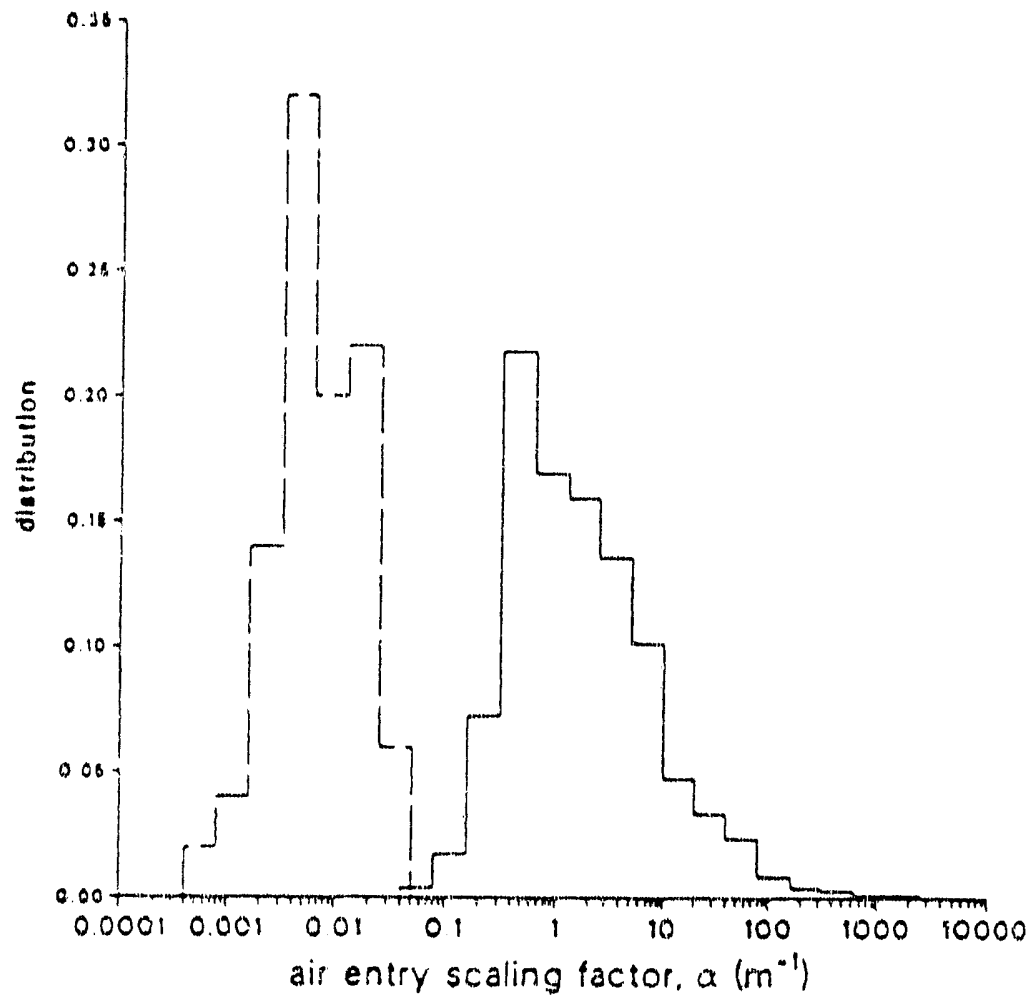

Figure 4-17 Distribution of air-entry scaling factor for natural soils and tuff matrix. 


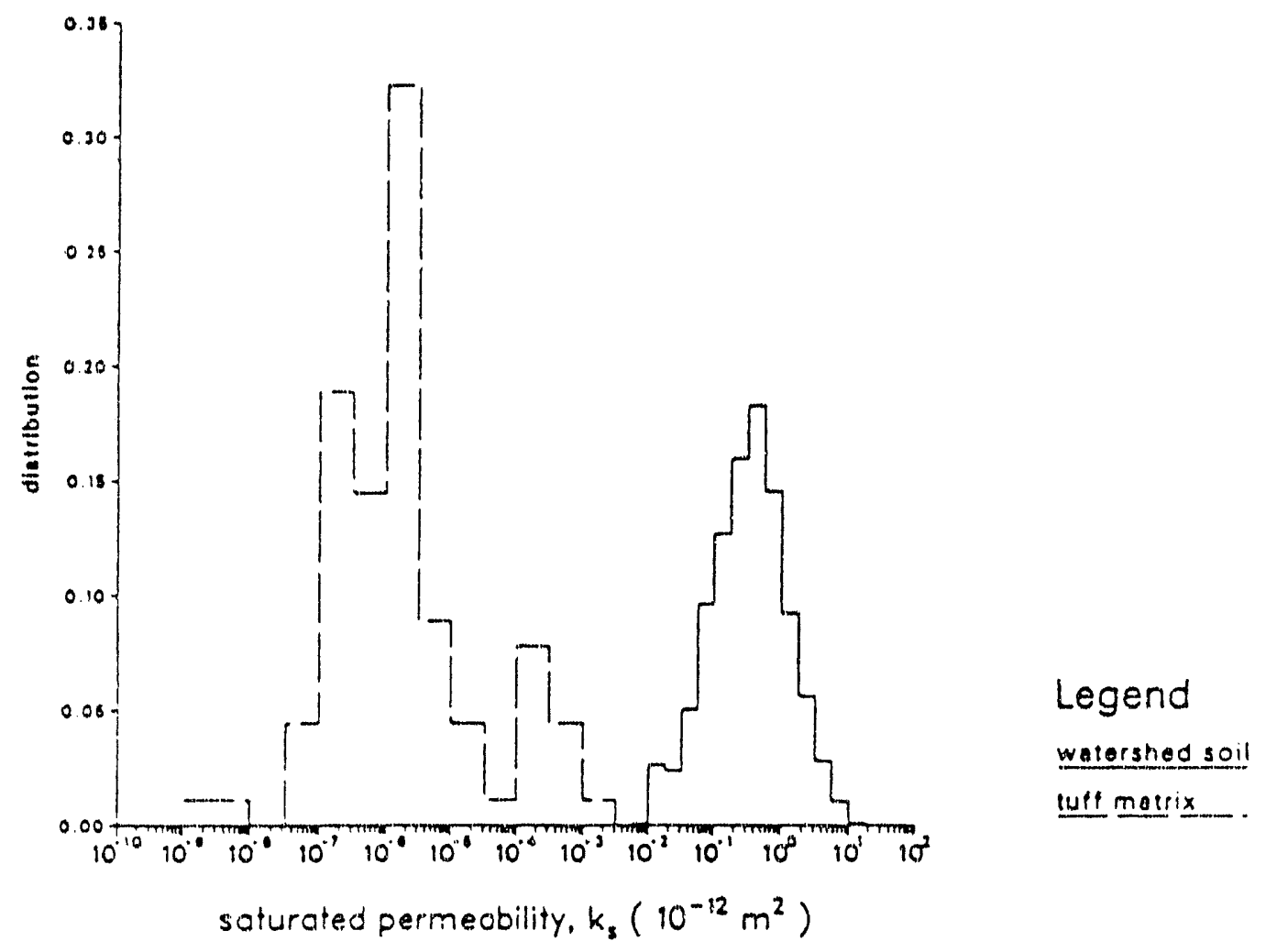

Figure 4-18 Distribution of saturated hydraulic conductivity for natural soils and cuff matrix. 


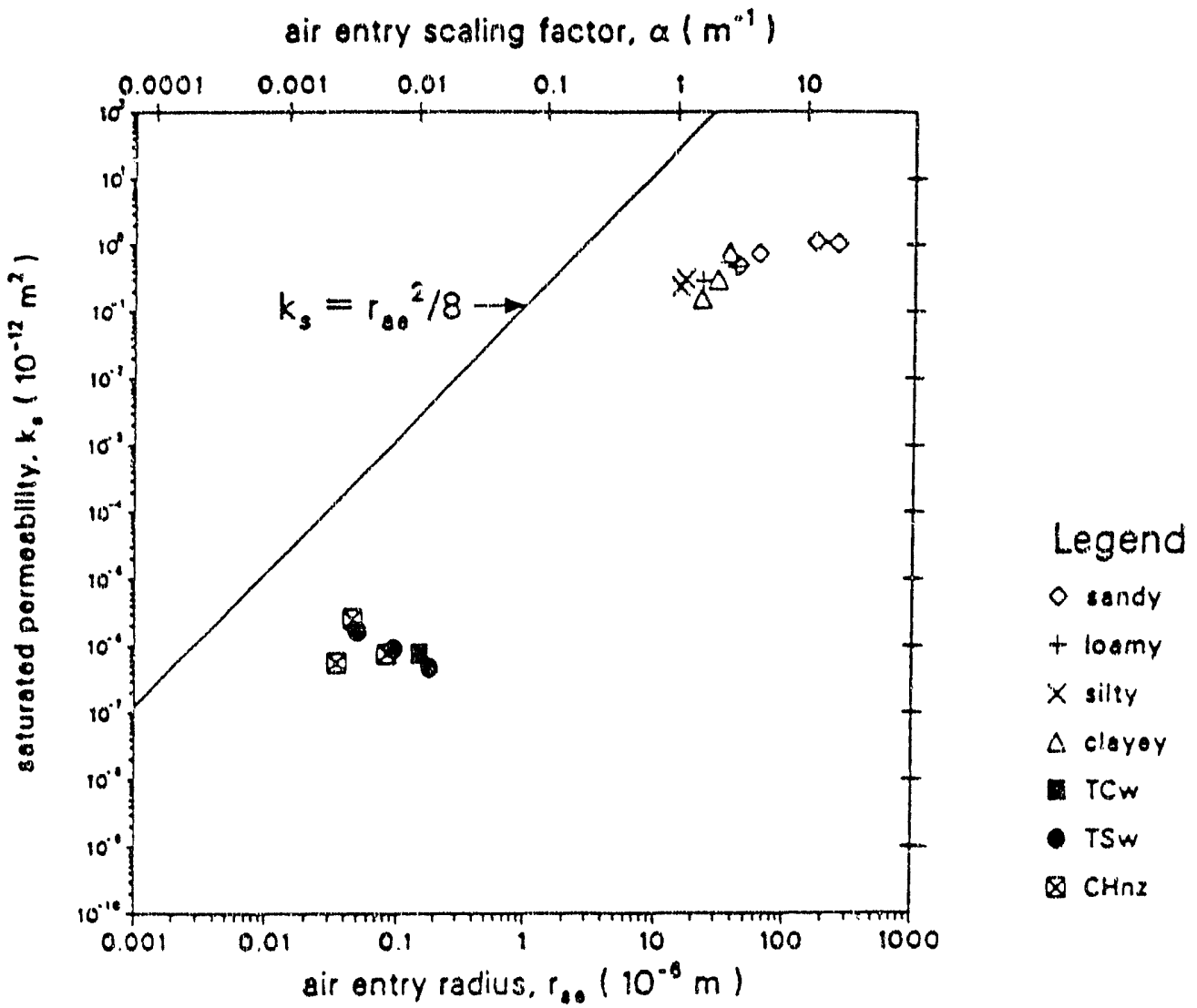

Figure 4-19 Correlation between saturated hydraulic conductivity and air-entry' scaling factor for natural soils and tuff matrix. 
through connected channels in a medium. The air entry radius measures the desaturation of fluid from the interior of medium through connected channels to the outside. Both parameters do not depend on dead end pores with stagnant fluid isolated from the connected channels. The porosity measures the volume of both connected channels and dead end pores. There is considerable interest in the literature on the permeability-porosity relationship. The correlation between permeability and air entry radius, observed in soil and tuff matrix data, also deserves attention in natural geological medium studies. Practically, this correlation of saturated permeability can be more easily demonstrated with air entry radius than with porosity. The air entry radius, or equivalently the air entry scaling factor, varies over several orders of magnitude among different geological media. The porosity varies over a much narrower range among different geological media. With wider variations, the air entry radius may correlate better with saturated permeabil. ity, which has the widest range of variation among all hydrological parameters.

\subsubsection{Fracture and Fault Parameters}

We need hydrologic data for the fractures and faults. There are only a few measurements on saturated permeability of fractured cores (Peters et al., 1984) and none on unsaturated characteristic curves of fractures. Only theoretical models have been derived or assumed to represent the fracture and fault characteristic curves. Wang and Narasimhan (1985) derived the fracture curves in terms of measurable bulk conductivity, fracture orientations and spacings, and fractions of fracture coatings. Peters et al. (1984) assumed that the fractures behave like permeable sand. Rulon et al. (1986) used a parallel channel model (Harrold et al. 1985) to derive anisotropic fracture-and fault characteristic curves. Figure 4-20 compares the model parameters in the correlation cross plot of saturated permeability and air entry radius. Clearly the model parameters are very scattered and some of the models have saturated permeability greater than the maximum capillary tuhe value $r_{a d}^{2} d 8$.

Even though theoretical models can be derived for fractures and faults, we need to have experimental measurements to validate the theoretical models. The contrast of hydrological parameters between fractures and tuff matrix in meter scale determines if the fluid flow is fast through fractures or slow through matrix under ambient partially saturated conditions. The contrast of hydrological parameters between stratigraphic units and bounding faults in kilometer scali: determines if the net infiltration is dispersed through the unsaturated tuff formations or concentrated through a few major faults from ground surface to water table. The lack of data in fraclures ard fauls is one of the reasons we survey the soil data and study the parameter variations 


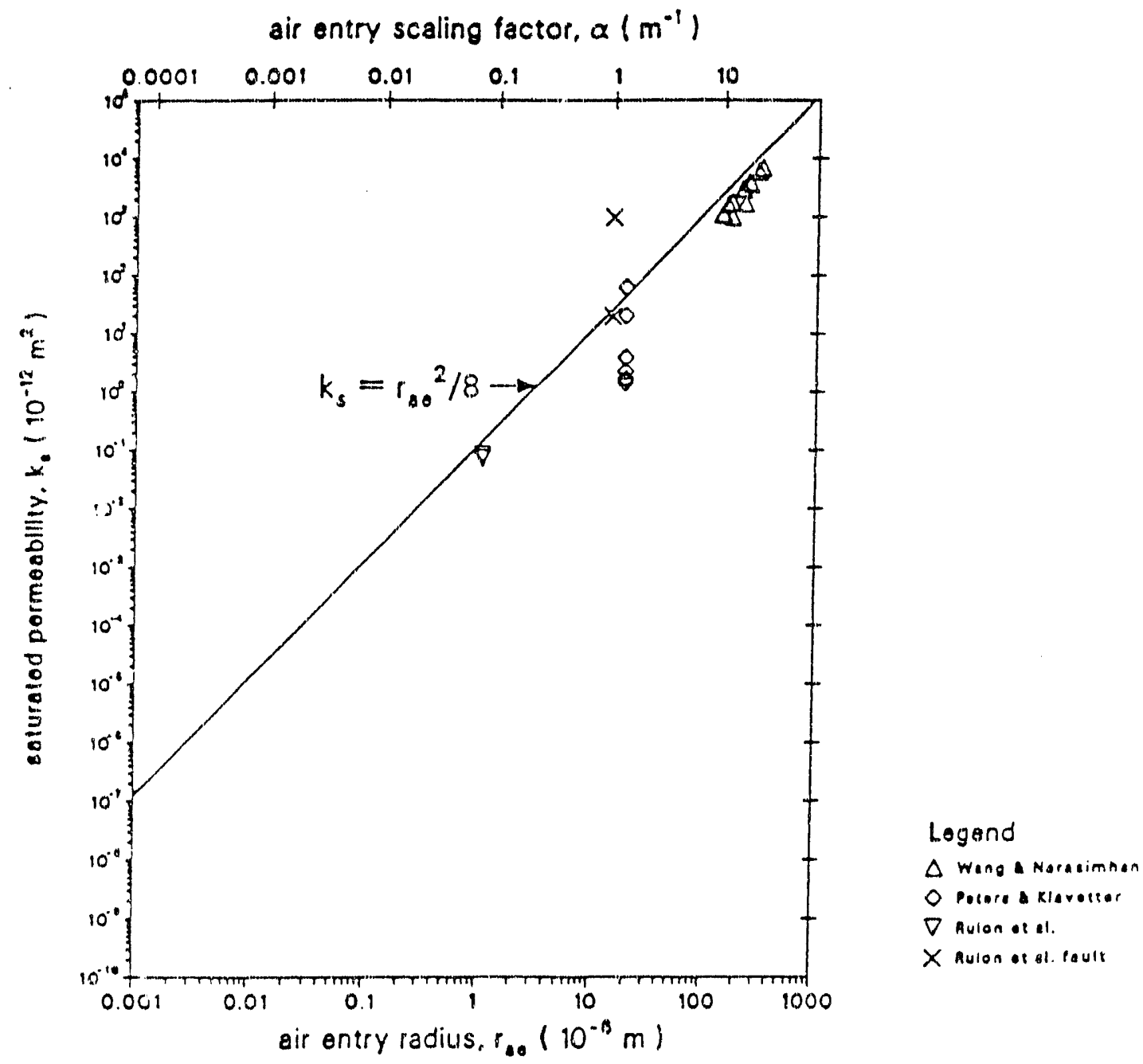

Figure 4-20 Correlation between saturated hydraulic conductivity and air-entry scaling factor for tuff fracture and fault models. 
and correlations among different natural geological media. If constraints and correlations can be shown to govern and scale among different geological media, we can evaluate whether the theoretical model parameters are reasonable and physical and establish better confidence in our calculation results.

If correlation between saturated permeability and air entry radius is important, we need to take this correlation into account. For stochastic modeling of alternating units of welded and nonwelded tuff or formation blocks bounded by fault zones, we propose to impose correlations and constraints on average values of saturated permeability and air entry scaling factor between highly different units to avoid unlikely combinations of parameters and predictions. Within a given stratigraphic unil, this physically meaningful correlation is likely to be masked by data variablily, and uncorrelated parameters with wide distributions may be acceptable.

\subsubsection{Fractal Theory: Spatial Correlation and Scaling Rough-Walled Fractures}

With parameter distributions and correlations determined from laboratory measurements, we need additional information on spatial correlation and scale dependance to properly model field-scalc hydrological processes. The spatial correlation structure determines the grid block sizes to represent a heterogeneous formation and the scaling law relates the laboratory measurements to field experiments. We have constructed a fractal fracture model (Wang et al., 1988) to represent a rough-walled fracture. From that particular model study, we also recognize that the fractal scaling approach can be used for other pa ameters. Even more important is the realization that fractal scaling is more general than traditional geostatistical analyses with empirical variograms for representation of spatial correlation.

A very useful and interesting aspect of the fractal is its scaling behavior. A rough profile measured with a low-resolution measuring device over a large distance may be geometrically similiar to the profile measured with a high-resolution device over a much smaller scale on the same rock sample. If this scaling property can indeed be demonstrated, we can deduce structure information at small scales by measuring at laige scales, and vice versa. The fractal dimension $D$ can be regarded as a scaling parameter. Figure 4-21 illustrates the steps in a recursive subdivision algorithm (Foumier et al., 1982; Brown, 19:7) to generate a fractal surface. The procedure starts with a 3 by 3 mesh with zern $z$ values on the mesh points to define a reference flat surface. In the second level with a 5 by 5 mesh, the $z$ value of a new point is computed by a Gaussian random variable function, with the expected value determined by its four known neightors (two on the 
boundaries) of the 3 by 3 mesh, and the standard deviation scaleci by the fractal dimension D and distance to the neighbors. We can continue the generation procedure level by level to finer mesh. A low-D surface will have the standard deviation scaled down faster than a high-D surface as one proceeds from one level to another level to fill the mesh.

We can use a similiar procedure to generate representations of heterogeneous media with spatially varying and correlated parameters. We simply replace the elevations by the property values and the Gaussian distribution by the parameter distribution.

If data values are known at a given number of locations, we can start with tr.ese locations as the first-level mesh points and generate refinements in accordance to the parameter distribution and spatial correlation structure. The heterogeneous field generated by this procedure will take into account the spatial correlation hecause the mean value at a mesh point is determined by the values of its neighbors. Becuase the generation of each sub-domain use the same procedure for the larger domain, domains of different sizes are scaled. We can have a low-level or lowresolution mesh to first model the field approximately and continue upgrading the results with meshes of higher-level refinements.

The fractal dimension $\mathrm{D}$ can be determined by log-log type curve analyses from variogram data of the parameter distribution. The log-log slope near the origin of zero spatial separation is equal to $6-2 \mathrm{D}$, as shown in Figure 4-22 from a derivation for the fractal fracture model. The spherical model and many other empirical variogram models widely used in geostatistical analyses have a log-log slope of 1 . If we reanalyze many variogram data in a log-log plot instead of linear plot, we can deduce the fractal dimension and use the fractal dimension as a scaling parameter to generate geostatistical representations.

If a heterogeneous ficld is generated with parameter distribution, correlation, and scaling properly taken into account, we can then proceed to solve flow equations to obtain the distributions in pressure, flux, and velocity field. We need to quantify uncertainties to obtain GWTT distributions. We are still perfecting our app:oaches and models so that the GWTT calculations can be efficiently and accurately calculated. 
Generation of $D=2.5$ Random Fractal Surface
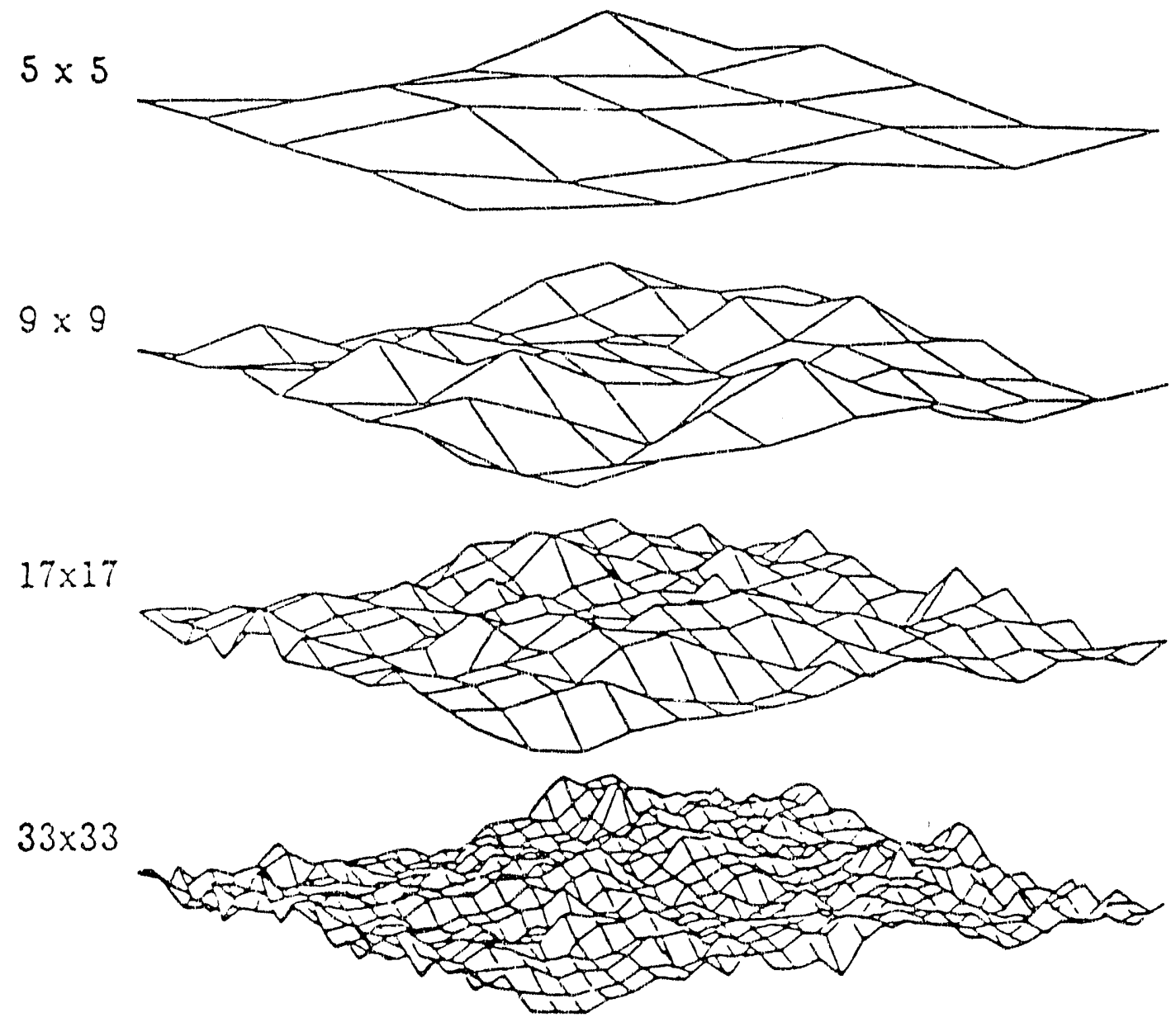

Figure 4.21 Steps involved in generating a rough fracture with fractal dimensions. 


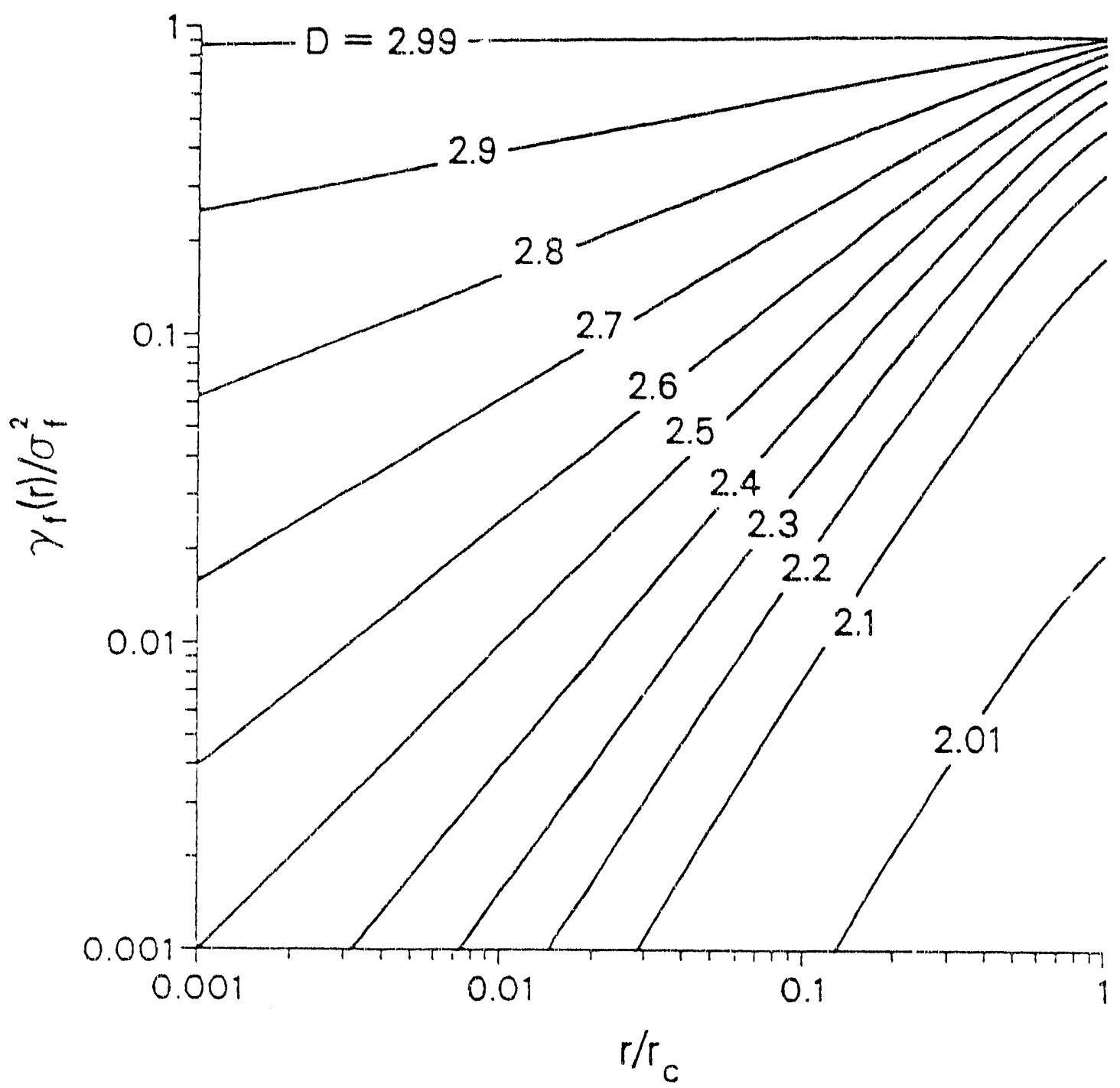

XBL $8711-4652$

Figure 4-22 Relation between variograms and fractal dimensions. 


\subsection{REFERENCES}

Bauer, S. J., 1986. "Preliminary Report on Yucca Mountain Fracture Characteristics," Memorandum to J. R. Tillerson dated March 27, 1986, Appendix D, Attachment 1 of the Site Characterization Plan Conceptual Design Report, SAND84-2641, compiled by H. R. MacDougall, L. W. Scully and J. R. Tillerson, Sandia National Laboratories, Albuquerque, NM (NN1.880902.0014-.0019).

Brooks, R. H. and A. T. Corey, 1966. "Properties of Porous Media Affecting Fluid Flow," J. Irrigation and Drainage Div., Proc. Am. Soc. Civ. Eng., 92, IR2, 61-88 (NNA.870407.0356).

Brown, S. R., 1987. "Fluid Flow Through Rock Joints: the Effect of Surface Roughness," J. Geophys. Res., 92, 1337-1347 (NNA..891106.0181).

Claassen, H. C. and A. F. White, 1979. "Application of Geochemical Kinetic Data to Groundwater Systems: A Tuffaceous-Rock System in Southern Nevada," in Chemical Modeling in Aqueous Systems, ACS Symposium Series No. 93, edited by E. A. Jenne, American Chemical Society, 771-793 (HQS.880517.174.2).

Davis, S. N., 1982. "Hydrogeology of Radioactive Waste Isolation: The Challenge of a Rational Assessment," in Recent Trends in Hydrogeology, edited by T. N. Narasimhan, Special Paper 189, Geological Society of America (NNA.900420.0081).

Davis, S. N. and E. Murphy, 1987. Dating Ground Water and the Evaluation of Repositories for Radioactive Waste, NUREG/CR-4912, U. S. Nuclear Regulatory Commission (NNA.900522.0260),

DOE, 1989. Site Characterization Plan Public Handbook, DOE/RW-0206, Office of Civilian Radioactive Waste Management Program, U. S. Department of Energy, Washington, DC (HQO.890103.0001).

Foumier, A., D. Fussell and L. Carpenter, 1982. "Computer Rendering of Stochastic Models," Comm. Asso. Computing Machinery, 25, 371-384 (NNA.891109.0129).

Genticr, S., 1986. Morphologie et Comportement Hydromecanique d'une Fracture Naturalle dans un Granite Sours Contrainte Normale, Doctoral Thesis, Univ. d'Orl'eans, France (NNA.900919.0188).

Harrold, P., C. C. Barton and P. Montazer, 1985. "Statistical Analysis of Fracture Roughness Profiles in Volcanic Tuffs, Yucca Mountain, Nevada," EOS, Trans. Amer. Geophys. Union. 66 (46), p. 883 (NNA.890315.0015). 
Holtan, H. N., C. B. England, G. P. Lawless and G. A. Schumaker, 1968. Moisture-Tension Data for Selected Soils on Experimental Watersheds, ARS 41-144, Agricultural Research Service, U. S. Department of Agriculture, Belisville, MD, 609 p. (NNA.901128.0167).

Klavetter, E. A. and R. R. Peters, 1907. An Evaluation of the Use of Mercury Porosimetry in Calculating Hydrologic Properties of Tuffs from Yucca Mountain, Nevada, SAND86-0286, Sandia National Laboratories, Albuquerque, NM (NNA.890327.0056).

Montazer, P. and W. E. Wilson, 1984. Conceptual Hydrologic Model of Flow in the Unsaturated Zone, Yucca Mountain, Nevada, USGS-WRI-84-4345. U. S. Geological Survey, Lakewood, CO, 55 p. (HQS.880517.1675).

Narasimhan, T N., 1987. "Some Thoughts on Model Verification," in Flow and Transport through Unsaturated Fractured Rock, Geophysical Monograph 42, American Geophysical Union, edited by D. D. Evans and T. J. Nicholson, 185-187 (NNA.900403.0386).

Narasimhan, T. N., 1985. "Geometry-Imbedded Darcy's Law and Transicnt Subsurface Flow," Water Resour. Res., 21, 1285-1292 (NNA.890315.0008)

Narasimhan, T. N. and P. A. Witherspoon?. 1976. "An Integrated Finite Difference Method for Analyzing Fluid Flow in Porous Media," Water Resour. Res., 12, 57-64 (NNA.890522.0253)

Narasimhan, T. N., P. A. Witherspoon and A. L. Edwards, 1978. "Numerical Model fc" Saturated-Unsaturated Flow in Deformable Media, 2. The Algorithm," Water Resour. Res., $14,255-261$ (NNA.890522.0216).

Oriz, T. S., R. L. Williams, F. B. Nimick, B. C. Whittet and D. L. South, 1984. A ThreeDimensional Model of Reference ThermallMechanical and Hydrological Stratigraphy at Yucca Mountain, Southern Nevada, SAND84-1076, Sandia National Laboratories, Albuquerque, NM (NNA.890315.(0)13).

Panian, T. F., 1987. Unsaturated Flow Properties Data Catalog. Volume II, DOE/NV/10384-20, Deser Research Institute, University of Nevada, 493 p. (NNA.890327.0061).

Peters, R. R. and E. A. Klavetter, 1988. "A Continuum Model for Water Movement in an Unsaturated Fractured Rock Mass," Water Resour. Res., 24, 416-430 (NNA.890523.0139).

Peters, R. R., E. A. KJavetter, I. J. Hall, S. C. Blair, P. R. Heller and G. W. Gee, 1984. Fracture and Matrix Hydrologic Characteristics of Tuffaceous Materials from Yucca Mountain, Nye County, Nevade SANu84-1471, Sandia National Laboratories, Albuquerque, NM, 188 p. (NNA.87(1)407.0036) 
Peters, R. R., E. A. Klavetter, J. T. George and J. H. Gauthier, 1987. "Measuring and Modeling Water Imbibition into Tuff," Proc. Symp. Flow and Transport through Unsaturated Fractured Rock, Geophysical Monograph 42, Am. Geophys. Union, edited by D. D. Evans and T. J. Nicholson, pp. 99-106 (NNA.900308.0324).

Pruess, K. and T. N. Narasimhan, 1985. "A Practical Method for Modeling Fluid and Heat Flow in Fractured Porous Media," Soc. Pet. Eng. J., 25, 14-26 (NNA.890522.0235).

Pruess, K., Y. W. Tsang and J. S. Y. Wang, 1985. "Modeling of Strongly Heat-Driven Flow in Partially Saturated Fractured Porous Media," Proc. Hydrogeology of Rocks of Low Permeability, Memoires 17, Int. Asso. Hydrogeologists, pp. 486-497 (NNA.890522.0270).

Roseboom Jr., E. H., 1983. Disposal of High-Level Nuclear Waste Above the Water Table in Arid Regions, Circular Number 903, U. S. Geological Survey (HQS.880517.2530).

Rulon, J., G. S. Bodvarsson and P. Montazer, 1986. Preliminary Numerica! Simulations of Groundwater Flow in the Unsaturated Zone, Yucca Mountain, Nevada, LBL-20553, Lawrence Berkeley Laboratory, Berkeley, CA, 91 p. (NNA.870908.0027).

Scott, R. B. and J. Bonk, 1984. Preliminary Geologic Map of Yucca Mountain, Nye County, Nevada, with Geologic Sections, USGS-OFR-84-494, U. S. Geological Survey, Denver, $\mathrm{CO}$ (HQS.880517.1443).

Scon, R. B., R. W. Spengler, S. Diehl, A. R. Lappin and M. P. Chornack, 1983. "Geologic Char. acter of Tuffs in the Unsaturated Zone at Yucca Mountain, Southern Nevada," in Role of the Unsaturated Zone in Radioactive and Hazardous Waste Disposal, edited by J. Mercer, P. S. Rao and I. W. Marine, Ann Arbor Science Publisher, Ann Arbor, MI, pp. 209-335 (NNA.870406.0034).

Sinnock, S., Y. T. Lin and J. T. Brannen, 1984. Preliminary Bounds on the Expected Postclosure Performance of the Yucca Mountain Repository Site, SAND84-1492. Sandia National Laboratories, Albuqueque, NM (HQS.880517.1460).

Spengler, R. W. and M. P. Chornack, 1984. Stratigraphic and Structural Characteristics of Volcanic Rocks in Core Hole USW-G4, Yucca Mountain, Nye County, Nevada, with a Section of Geophysical Logs by D. C. Muiler and J. E. Kibler, USGS-OFR-84-789, U. S. Geological Survey, Denver, CO (NNA.870519.0076).

Thordarson, W., 1983. Geohydrologic Data and Test Results from Well J-13, Nevada Test Site, Nye County, Nevada, USGS-WRI-83-4171, U. S. Geological Survey, Denver, CO (HQS.880517.1849). 
Travis, B. J., S. W. Hodson, H. E. Nuttall, T. L. Cook and R. S. Runuberg, 1984. "Numerical Simulation of Flow and Transport in Fractured ruff," Proc. Scientific Basis for Nuclear Waste Management VII, Mat. Res. Soc. Sym. Proc., 26, 1039-1046 ( NA.890522.0239).

"Tsang, Y. W., 1984. "The Effect of Tortuosity on Fluid Flow Through a Single Fracture," Water Resour. Res., 20, 1209-1215 (NNA.900420.0082).

van Genuchten, M. Th. 1980. "A Closed-Form Equation for Predicting Hydraulic Conductivity of Unsaturated Soils," Soil Sci. Soc. Am. J., 44, 892-898 (NNA.890522.0287).

Wang, J. S. Y. and T. N. Narasimhan, 1988. Hydrologic Modeling of Vertical and Laternl Movement of Partially Saturated Fluid" Flow near a Fault Zone at Yucca Mountain, SAND87. 7070, Sandia National Laboratories, Albuquerque, NM, LBL-23510, Lawrence Berkeley Laboratory, Berkeley, CA, 122 p. (NNA.871109.0057).

Wang, J. S. Y. and T. N. Narasimhan, 1980. Hydrologic Mechanisms Governing Partially Saturated Fluid Flow in Fracured Welded Units and Porous Non-welded Units at Yucca Mountain, SAND85-1114, Sandia National Laboratories, Albuquerque, NM, LBL-21022, Lawrence Berkeley Laboratory, Berkeley, CA, 126 p. (NNA.870806.0363).

Wang. J. S. Y. and T. N. Narasimhan, 1985. "Hydrologic Mechanisms Governing Fluid Flow in a Partially Saturated, Fractured, Porous Medium," Water Resour. Res., 21, 1861-1874 (NNA.891109.0050).

Wang. J. S. Y, and T. N. Narasimhan, 1984. Hydrologic Mechanisms Governing Fluid Flow in a Partially Saturated, Fractured, Porous Tuff at Yucca Mountain, SAND84.7202, Sandiat National Laboratories, Albuquerque, NM, LBL-18473, Lawrence Berkeley Laboratory', Berkeley, CA (NNA.870407.0209).

Wang, J. S. Y., T. N. Narasimhan and C. H. Scholz, 1988. "Aperture Correlation of a Fractal Fracture," J. Geophy's. Res. 93, 2216-2224 (NNA.891222.0005).

Wecks, E. P., 1987. "Effect of Topography on Gas Flow in Unsaturated Fractured Rock: Con. cepts and Observations." in Flow and Transport through Ursaturated Fractured Rock. Geophysical Monograph 42, American Geophysical Union, edited by D. D. Evans and T. J. Nicholson, 165-170 (HQS.880517.3214).

White, A. F. and A. Y'ee, 1986. "Near-Surface Alkali Diffusion into Glassy and Crystalline Siticates at $25^{\circ} \mathrm{C}$ to $1000^{\circ} \mathrm{C}$." Geochemical Processes at Mineral Surfaces, American Chemical Saciety Symposium Series 323, edited by J. A. Davis, 587-598 (NNA.900917.0130). 
Winograd, I. J., 1986. Archaeology and Public Perception of a Transscienific ProblemDisposal of Toxic Wastes in the Unsaturated Zune, USGS-OFR-86-136, U. S. Geological Survey (NNA.900228.0117).

Winograd. I. J., 1981 "Radioactive Waste Disposal in Thick Unsaturated Zones," Science, 212 (4502), 1457-1464 (HQS.880517.2584).

Winograd, 1. J., 1976. "Reply to Commentary on 'Radioactive Waste Storage in the Arid Zone'," EOS, Trans. Amer. Geophys. Uniom, 57, 215-216 (NNA.900420.0084).

Winograd, 1. J., 1974. "Radioactive Waste Storage in the Arid Zone," EOS, Trans. Amer. Geophys. Union, 55, 884-894 (NNA.900420.0083).

Winograd, I. J., 1972. "Near Surface Storage of Solidifi.d High-Level Radioactive Wastes in Thick (400-2,000 foot) Unsaturated Zones in the Southwest," Abstracts with Progrcums, 4, Geological Society of Amer., p. 708 (NNA.900522.0262). 


\title{
6.0 APPENDIX
}

\section{Information from the Reference Information Base}

\section{Used in this Report}

This report contains no information from the Reference Information Base.

Candidate Information for the

Refe:"ence Information Base

This report contains no candidate information for the Reference Information Base.

\author{
Candidate Information \\ for the
}

Site and Engineering Properties Data Base

This report contains no candidate information for the Site and Engineering Properties Data Base. 


\section{DISTRIBUTION LIST}

1 J. W. Bartlett, Director (RW-1)

Office of Civilian Radioactive

Waste Management

U.S. Department of Energy

1000 Independence Avenue, S.W.

Washington, DC 20505

1 F. G. Peters, Deputy Director (RW-2) 1

Office of Civilian Radioactive

Waste Management

U.S. Department of Energy

1000 Independence Avenue, S.W

Washington, DC. 20585

1 T. H. Isaacs (RW-4)

Office of Strategic Planning and International Programs

Office of Civilian Radioactive Waste Management

U.S. Department of Energy

1000 Independence Avenue, S.W.

Washington, DC 20585

1 J. D. Saltzman (RW-5)

Office of External Relations

Office of Civilian Radioactive Waste Management

U.S. Department of Energy

1000 Independence Avenue, S.W.

Washington, DC 20585

1 Samuel Rousso (RW-10)

Office of Program and Resources Management

Office of Civilian Radioactive Waste Management

U.S. Department of Energy

1000 Independence Avenue, S.W.

Washington, DC 20585
1 J. C. Bresee (RW-10)

Office of Civilian Radioactive

Waste Management

U.S. Department of Energy

1000 Independence Avenue, S.W.

Washington, DC 20585

C. P. Gertz (RW-20)

Office of Geologic Disposal

Office of Civilian Radioactive

Waste Management

U.S. Department of Energy 1000 Independence Avenue, S.W.

Washington, DC 20585

1 S. J. Brocoum (RW-22)

Analysis and Verification Division

Office of Civilian Radioactive

Waste Management

U.S. Department of Energy

1000 Independence Avenue, S.W.

Washington, DC 20585

1 J. Roberts, Acting Assoc. Dir. (RW.30)

Office of Systems and Compliance

Office of Civilian Radioactive Waste Management

U.S. Department of Energy

1000 Independence Avenue, S.W.

Washington, DC 20585

1 J. Roberts (RW-33)

Director, Regulatory Compliance Division

Office of Civilian Radioactive Waste Management

U.S. Department of Energy

1000 Independence Avenue, S.W.

Washington, DC 20585 
1 G. J. Parker (RW-332)

Office of Civilian Radioactive Waste Management

U.S. Department of Energy

1000 Independence Avenue, S.W.

Washington, DC 20585

1 R. A. Milner (RW-40)

Office of Storage \& Transportation

Office of Civilian Radioactive Waste Management

U.S. Department of Energy

1000 Independence Avenue, S.W.

Washington, DC 20585

1 S. Rousso, Acting Assoc. Director (RW-50)

Office of Contract Business Management

Office of Civilian Radioactive Waste Management

U.S. Department of Energy 1000 Independence Avenue, S.W. Washington, DC 20585

1 Trudy Wood (RW-52)

Director, M\&O Management Div. Office of Civilian Radioactive Waste Management U.S. Department of Energy 1000 Independence Avenue, S.W. Washington, DC 20585

1 Clarence R. Allen

Nuclear Waste Technical Review Board

1000 E. California Blvd.

Pasadena, CA 91106

1 John E. Cantlo., Chairman

Nuclear Waste Technical Review Board

1795 Bramble Dr.

East Lansing, MI 48823
1 Garry D. Brewer

Nuclear Waste Technical Review Board

Univ. of Michigan

Dana Bldg., Rm 3516

Ann Arbor, Michigan 48109-1115

1 Pat Domenico

Nuclear Waste Technical Review Board

1100 Wilson Boulevard, Suite 910

Arlington, VA 22209

1 Donald Langmuir

Nuclear Waste Technical

Review Board

109 So. Lookout Mountain Cr.

Golden, CO 80401

1 John J. McKetta

Nuclear Waste Technical

Review Board

Dept. of Chemical Engineering

CRE Bldg. 1.450

Austin, TX 78712-1062

1 D. Warner North

Nuclear Waste Technical

Review Board

Decision Focus, Inc.

4984. El Camino Real

Los Altos, CA 94062

1 Dennis L. Price

Nuclear Wast Technical

Review Board

1011 Evergreen Way

Blacksburg, VA 24060

1 Ellis D. Verink

Nuclear Waste Technical Review Board

4401. N.W. 18th Place

Gainesville, FL 32605 
5 C. P. Gertz, Project Manager Yucca Mountain Site Characterization Project Office

U.S. Department of Energy

P.O. Box 98608--MS 523

Las Vegas, NV 89193-8608

1 C. L. West, Director Office of External Affairs DOE Field Office, Nevada U.S. Department of Energy P.O. Box 98518 Las Vegas, NV 89193-8518

12 Technical Information Officer DOE Nevada Field Office U.S. Department of Energy P.O. Box 98518 Las Vegas, NV 89193-8518

1 P. K. Fitzsimmons, Technical Advisor

Office of Assistant Manager for Environmental Safety and Health DOE Field Office, Nevada U.S. Department of Energy P.O. Box 98518

Las Vegas, NV 89193-8518

1 D. R. Elle, Director Environmental Protection Division DOE Nevada Field Office U.S. Department of Energy P.O. Box 98518 Las Vegas, NV 89193-8518

1 Repository Licensing \& Quality Assurance Project Directorate Division of Waste Management U.S. Nuclear Regulatory Commission Washington, DC 20555
1 Senior Project Manager for Yucca Mountain

Repository Project Branch

Division of Waste Management

U.S. Nuclear Regulatory Commission

Washington, DC 20555

1 NRC Document Control Desk

Division of Waste Management

U.S. Nuclear Regulatory Commission

Washington, DC 20555

1 P. T. Prestholt

NRC Site Representative

301 E. Stewart Ave., Room 203

Las Vegas, NV 89101

1 E. P. Binnall

Field Systems Group Leader

Building 50B/4235

Lawrence Berkeley Laboratory

Berkeley, CA 94720

1 Center for Nuclear Waste

Regulatory Analyses

6220 Culebra Road

Drawer 28510

San Antonio, TX 78284

3 W. L. Clarke

Technical Project Officer for YMP

Attn: YMP/LRC

Lawrence Livermore National Laboratory

P.O. Box 5514

Livermore, CA 94551 
1 J. A. Blink

Deputy Project Leader

Lawrence Livermore National Laboratory

Mail Stop 527

101 Convention Center Drive

Suite 280

Las Vegas, NV 89109

4 J. Canepa

Technical Project Officer for YMP

N-5, Mail Stop J521

Los Alamos National Laboratory

P.O. Box 1663

Los Alamos, NM 87545

1 H. N. Kalia

Exploratory Shaft Test Manager

Los Alamos National Laboratory

Mail Stop 527

101 Convention Center Dr

Suite 820

Las Vegas, NV 89109

1 J. F. Divine

Assistant Director for

Engineering Geology

U.S. Geological Survey

106 National Center

12201 Sunrise Valley Dr.

Reston, VA 22092

6 L.. R. Hayes

Technical Project Officer

Yucca Mountain Project Branch

MS 425

U.S. Geological Survey

P.O. Box 25046

Denver, CO 80225
1 V. R. Schneider

Asst. Chief Hydrologist--MS 414

Office of Program Coordination \&

Technical Support

U.S. Geological Survey

12201 Sunrise Valley Drive

Reston, VA 22092

1 J. S. Stuckless

Geological Division Coordinator

MS 913

Yucca Mountain Project

U.S. Geological Survey

P.O. Box 25046

Denver, CO 80225

1 D. H. Appel, Chief

Hydrologic Investigations Program

MS 421

U.S. Geological Survey

P.O. Box 25046

Denver, CO 80225

1 E. J. Helley

Branch of Western Regional Geology

MS 427

U.S. Geological Survey

345 Middlefield Road

Menlo Park, CA 94025

1 R. W. Craig, Chief

Nevada Operations Office

U.S. Geological Survey

101 Convention Center Drive

Suite 860, MS 509

Las Vegas, NV 89109

1 D. Zesiger

U.S. Geological Survey

101 Convention Center Dr.

Suite 860 - MS 509

Las Vegas, NV 89109 
1 G. L. Ducret, Associate Chief Yucca Mountain Project Division U.S. Geological Survey P.O. Box 25046 421 Federal Center Denver, CO 80225

1 A. L. Flint U.S. Geological Survey MS 721

P.O. Box 327

Mercury, NV 89023

1 D. A. Beck U.S. Geological Survey 1500 E. Tropicana, Suite 201 Las Vegas, NV 89119

1 P. A. Glancy

U.S. Geological Survey Federal Building, Room 224

Carson City, NV 89701

1 Sherman S. C. Wu Branch of Astrogeology U.S. Geological Survey 2255 N. Gemini Dr. Flagstaff, AZ 86.001

1 J. H. Sass

Branch of Tectonophysics U.S. Geological Survey 2255 N. Gemini Dr.

Flagstaff, AZ 86001

1 DeWayne A. Campbell Technical Project Officer for YMP U.S. Bureau of Reclamation Code D-3790 P.O. Box 25007 Denver, CO 80225
1 J. M. LaMonaca

Records Specialist

U.S. Geological Survey

421 Federal Center

P.O. Box 25046

Denver, CO 80225

1 W. R. Keefer

U.S. Geological Survey

913 Federal Center

P.O. Box 25046

Denver, CO 80225

1 M. D. Voegele

Technical Project Officer for YMP

Science Applications International Corp.

101 Convention Center Dr.

Suite 407

Las Vegas, NV 89109

2 L. D. Foust

Nevada Site Manager

TRW Environmental Safety Systems 101 Convention Center Drive Suite 540, MS 423

Las Vegas, NV 89109

1 C. E. Ezra

YMP Support Project Manager

EG\&G Energy Measurements, Inc.

MS V-02

P.O. Box 1912

Las Vegas, NV 89125

1 E. L. Snow, Program Manager Roy F. Weston, Inc.

955 L'Enfant Plaza, Southwest Washington, DC 20024

1 Technical Information Center Roy F. Weston, Inc. 955 L'Enfant Plaza, Southwest Washington, DC 20024 
1 D. Hedges, Vice President, Quality Assurance Roy F. Weston, Inc. 4425 Spring Mountain Road Suite 300

Las Vegas, NV 89102

1 D. L. Fraser, General Manager

Reynolds Electrical \& Engineering Company

Mail Stop 555

P.O. Box 98521

Las Vegas, NV 89193-8521

1 R. F. Pritchett

Technical Project Officer for YMP Reynolds Electrical \& Engineering Company

MS 408

P.(). Box 98521

Las Vegas, NV 89193-8521

1 B. W. Colston

President/General Manager

Las Vegas Branch

Raytheon Services Nevada

MS 416

P.O. Box 95487

Las Vegas, NV 89193-5487

1 R. L. Bullock

Technical Project Officer for YMP

Raytheon Services Nevada

Suite P250, MS 403

101 Convention Center Dr.

Las Vegas, NV 84109

1 Paul Eslinger, Manager

PASS Program

Pacific Northwest Laboratory

P.O. Box 999

Richland, WA 99352
1 A. T. Tamura

Science and Technology Division

Office of Scientific and Technical

Information

U.S. Department of Energy

P.O. Box 62

Oak Ridge, TN 37831

1 Carlos G. Bell, Jr.

Professor of Civil Engineering Civil and Mechanical Engineering Department

University of Nevada, Las Vegas

4505 South Maryland Parkway

Las Vegas, NV 89193-3478

1 P. J. Weecien, Acting Director

Nuclear Radiation Assessment Division

U.S. Environmental Protection Agency

Environmental Monitoring Systems Laboratory

P.O. Box 93478

Las Vegas, NV 89193-3478

1 ONWI Library

Battelle Columbus Laboratory

Office of Nuclear Waste Isolation

505 King: Avenue

Columbus, $\mathrm{OH} 43201$

1 T. Hay, Executive Assistant

Office of the Governor

State of Nevada

Capitol Complex

Carson C.ity, NV 89710 
3 R. R. Loux, Jr.

Executive Director

Nuclear Waste Project Office

State of Nevada

Evergreen Center, Suite 252

1802 North Carson Street

Carson City, NV 89710

1 C. H. Johnson

Technical Program Manager

Nuclear Waste Project Office

State of Nevada

Evergreen Center, Suite 252

1802 North Carson Street

Carson City, NV 89710

1 John Fordham

Water Resources Center

Desert Research Institute

P.O. Box 60220

Reno, NV 89506

1 David Rhode

Desert Research Institute

P.O. Box 60220

Reno, NV 89506

1. Eric Anderson

Mountain West Research-

Southwest Inc.

2901 N. Central Ave. \#1000

Phoenix, AZ 85012-2730

1 Department of Comprehensive Planning

Clark County

225 Bridger Avenue, 7th Floor

Las Vegas, NV 89155

1 Planning Department

Nye County

P.O. Box 153

Tonopah, NV 89049
1 Lincoln County Commission

Lincoln County

P.O. Box 90

Pioche, NV 89043

5 Judy Foremaster

City of Caliente

P.O. Box 158

Caliente, NV 89008

1 Economic Development

Department

City of Las Vegas

400 East Stewart Avenue

Las Vegas, NV 89101

1 Community Planning \&

Development

City of North Las Vegas

P.O. Box 4086

North Las Vegas, NV 89030

1 Director of Community Planning

City of Boulder City

P.O. Box 367

Boulder City, NV 89005

1 Commission of the European Communities

200 Rue de la Loi

B-1049 Brussels

BELGIUM

2 M. J. Dorsey, Librarian

YMP Research and Study Center

Reynolds Electrical \& Engineering

Co., Inc.

MS 407

P.O. Box 98521

Las Vegas, NV 89193-8521 
1 Amy Anderson

Argonne National Laboratory

Building 362

9700 So. Cass Ave.

Argonne, IL 60439

1 Steve Bradhurst

P.O. Box 1510

Reno, NV 89505

1 Vernon Poe

P.O. Box 1026

Hawthorne, NV 89415

1 Jason Pitts

Lincoln County Courthouse

Pioche, NV 89043

1 Michael L. Baughman

35 Clark Road

Fiskdale, MA 01518

1 Glenn Van Roekel

Director of Community

Development

City of Caliente

P.O. Box 158

Caliente, NV 89008

1 Ray Williams, Jr.

P.O. Box 10

Austin, NV 89310

1 Leonard J. Fiorenzi

P.O. Box 257

Eureka, NV 89316

1 Juanita Hayes

P.O. Box 490

Goldfield, NV 89013

1 Bjorn Selinder

190 W. First St.

Fallon, NV 89406
1 Charles Thistlethwaite, AICP

Associate Planner

Planning Department

Drawer L

Independence, CA 93526

1 Richard Codell

U.S. Nuclear Regulatory

Commission

M/S 4-H-3

Washington, DC 20555

1 Les Bradshaw

Nye County District Attorney

P.O. Box 593

Tonopah, NV 89049

1 Seth M. Coplan

U.S. Nuclear Regulatory

Commission

$\mathrm{M} / \mathrm{S} 4-\mathrm{H}-3$

Washington, DC 20555

1 Norman A. Eisenberg

U.S. Nuclear Regulatory

Commission

$\mathrm{M} / \mathrm{S} 4-\mathrm{H}-3$

Washington, DC 20555

1 A. B. Gureghian

CNWRA

6220 Culebra Road

San Antonio, TX 78228-0510

1 Phil Justus

U. S. Nuclear Regulatory

Commission

M/S 4-H-3

Washington, DC 20555 
1 Johan Andersson

SKI

Division of Nuclear Waste

Sehlstedtgatan II

Box 27106

S-102 52 Stockholm

SWEDEN

1 Michael J. Apted

Intera Sciences

3609 S. Wadsworth Blvd., 5th Fl.

Denver, CO 80235

1 Daniel B. Bullen

Iowa State University

P.O. Box 1768

Ames, IA 50010

1 Kevin Coppersmith

Geomatrix Consutants

100 Pine Street, 10th Floor

San Francisco, CA 94111

1 Allen G. Croff

Associate Director of Waste \&

Environmental Programs

Chemical Technology Division

Oak Ridge National Laboratory

Oak Ridge, T'N 37831

2 A. L. Dudley

SPECTRA Research Institute

1603 University NE

Albuquerque, NM 87102

1 M.E. Harr

School of Civil Engineering

Pudue University

1284 Civing Engineering Building

West Lafayette, IN 47907-1284
1. Edwin E. Kinter

Bradley Hill Road

P.O. Box 682

Norwich, VT 05055

1 Robert Luce

Nuclear Waste Technical

Review Board

1100 Wilson Blvd., Suite 910

Arlington, VA 22209

1 Edward A. Mason

Amoco Resource Center

P.O. Box 451

46 Admirals Lane

Osterville, MA 02655-0451

1 Russell McFarland

Nuclear 'Waste Technical

Review Board

1100 Wilson Blvdi, Suite 910

Arlington, VA 22209

1 Robin McGuire

Risk Engineering, Inc.

5255 Pine Ridge Road

Golden, CO 80403

1 Ian McKinley

NAGRA

Hardstrasse 73

Wettingen, $\mathrm{CH}-5430$

SWITZERLAND

1 Fred W. McLafferty

Department of Chemistry

Cornell University

2600 Baker Laboratory

Ithaca, NY 14853-1301 
1 Brad R. Mettam

Project Coordinator

County of Inyo

Yucca Mountain Repository

Assessment Office

P.O. Drawer L

Independence, CA 93526

1 Ian Miller

Golder Associates, Inc.

4104 148th Avenue, N.E.

Redmond, WA 98052

1 F. Joseph Pearson, Jr.

1304 Walnut Hill La.

Suite 210

Irving, TX 75038

1 T. H. Pigford

University of California

Department of Nuclear

Engineering

Berkeley, C.A 94720

1 Norman C. Rasmussen

Massachusetts Institute of Technologry

Department of Nuclear

Engineering

Bldg. 24-205

Cambridge, MA 02139

1 Leon Reiter

Nuclear Waste Technical Review Board

1100 Wilson Blvd., Suite 910

Arlington, VA 22209-2297

1 Larry Rickertsen

TRW Envirommental Safety Systems

2650 Park Tower Drive

Fairfax, VA 22033
1 Benjamin Ross

Disposal Safety, Inc.

1660 L Street, N.W., Suite 314

Washington, DC 20036

1 Frank W. Schwartz

195 Thornbury La.

Powell, OH 43065

1 Robert Shaw

Electric Power Research Institute 3412 Hillview Avenue

Palo Alto, CA 94304

1 Roger Staehle

University of Minnesota

Dept. of Chemical Engineering and Materials Science

22. Red Fox Road

North Oaks, IMN 55127

1 Jane Stockey

U.S. Department of Energy

Forrestal RW-22, 7F-091/FORS

Washington, DC 20555

1 K. T. Thomas

National Academy of Science

2001 Wisconsin Ave., N.W.

Harris Bldg., Room 456

Washington, DC 20007

1 Robert F. Williams

EPRI, Nuclear Power Div.

P.O. Box 10412

3412 Hillview Ave.

Palo Alto, CA 94303

1 Tom Bjerstedt

Yucca Mountain Project Office

U.S. Department of Energy

P.O. Box 98608

$\mathrm{M} / \mathrm{S} 523$

Las Vegas, NV 89193-8518 
1 Maxwell Blanchard

Yucca Mountain Project Office

P.O. Box 98608

$\mathrm{M} / \mathrm{S} 523$

Las Vegas, NV 891.93-8518

5 Jeremy Boak

Yucca Mountain Project Office

U.S. Department of Energy

P.O. Box 98608

M/S 523

Las Vegas, NV 89193-8518

1 Scott Borg

Yucca Mountain Project Office

U.S. Department of Energy

P.O. Box 98608

$\mathrm{M} / \mathrm{S} 523$

Las Vegas, NV 89193-8518

1 J. Russell Dyer

Yucca Mountain Project Office

U.S. Department of Energy

P.O. Box 98608

$\mathrm{M} / \mathrm{S} 523$

Las Vegas, NV 89193-8518

1 Claudia Newbury

Yucca Mountain Project Office

U.S. Department of Energy

P.O. Box 98608

$\mathrm{M} / \mathrm{S} 523$

Las Vegas, NV 89193-8518

1 Ardyth Simmons

Yucca Mountain Project Office

U.S. Department of Energy

P.O. Box 98608

$\mathrm{M} / \mathrm{S} 523$

Las Vegas, NV 89193-8518
1 Scott Sinnock

TRW Environmental Safety

Sy'stems

101 Convention Center Drive

Suite P110

Las Vegas, NV 89109

1 Jean Younker

TRW Environmental Safety

Systems

101 Convention Center Drive Suite P110

Las Vegas, NV 89109

1 R. W. Nelson

IN'TERA-M\&O

101 Convention Center Drive Suite P110

Las Vegas, NV 89109

1 Suresh Pahwa

INTERA

6850 Austin Center Blvd.

Suite 300

Austin, TX 78731

1 Abraham Van Luik

INTERA-M\&O

101 Convention C'enter Drive Suite \# P110

Las Vegas, NV 89109

1 W. W.-L.. Lee

Lawrence Berkeley Laboratory

Earth Sciences Division

1 Cyclotron Road

Berkeley, CA 94720

10 T. N. Narasimhan

Lawrence Berkeley Laboratory

Earth Sciences Division

1 Cyclotron Road

Berkeley, C.A 94720 
1 Karsten Pruess

Lawrence Berkeley Laboratory

Earth Sciences Division

1 Cyclotron Road

Berkeley, CA 94720

1 C. F. Tsang

Lawrence Berkeley Laboratory

Earth Sciences Division

1 Cyclotron Road

Berkeley, CA 94720

10 Joseph Wang

Law'rence Berkeley Laboratory

Earth Sciences Division

1 Cyclotron Road

Berkeley, CA 94'720

1 Thomas A. Buscileck

Lawrence Livermore National Laboratory

P.O. Box 308

$\mathrm{M} / \mathrm{SL} 206$

Livermore, CA 94550

1 Dwayne Chesnut

Lawrence Livermore National Laboratory

P.O. Box 808

$\mathrm{M} / \mathrm{SL202}$

Livermore, CA 94551

i William G. Halsey

Lawrence Livermore National

Laboratory

P.O. Box 808

M/S L-204

Livermore, $C_{4} 44551$
1 Arnette Macintyre

Lawrence Livermore National

Laboratory

P.O. Box 808

$\mathrm{M} / \mathrm{S} \mathrm{L}-194$

Livermore, CA 94551

1 Williarn J. O'Connell

Lawrence Livermore National

Laboratory

P.O. Box 808

M/S L-195

Livermore, CA 94551

1 Michael Revelli

Lawrence Livermore National

Laboratory

P.O. Box 808

M/S L-206

Livermore, CA 94551

1 Richard Van Konynenburg

Lawrence Livermore National Laboratory

P.O. Box 808

Livermore, CA 94551

1 Bruce M. Crowe

Los Alamos National

Laboratory/LV

101 Convention Center Drive

Suite 820

Las Vegas, NV 89109

1 Arend Meijer

Los Alamos National Laboratory

P.O. Box 1663

M/S J514

Los Alamos, NM 87545 
1 Everett Springer

Los Alamos National Laboratory

P.O. Box 1663

$\mathrm{M} / \mathrm{S} \mathrm{J495}$

Los Alamos, NM 87545

1 Aaron Thode

Los Alamos National Laboratory

$\mathrm{M} / \mathrm{S}$ F 607

P.O. Box 1663

Los Alamos, NM 87545

1 Greg Valentine

Los Alamos National Laboratory

P.O. Box 1663

$\mathrm{M} / \mathrm{S}$ F665

Los Alamos, NM 87545

1 L. A. Doremus

Pacific Northwest Laboratory

P.O. Box 999

M/S K6-96

Richland, WA 99352

1 D. W. Engel

Pacific Northwest Laboratory

P.O. Box 999

M/S K7-34

Richland, WA 99352

1 T. B. Miley

Pacific Northwest Laboratory

P.O. Box 999

$\mathrm{M} / \mathrm{S} \mathrm{K7}$.34

Richland, WA 99,352

1 M. T. Murphy

Pacific Northwest Laboratory

P.O. Box 999

$\mathrm{M} / \mathrm{S}$ K6-84

Richland, WA 99352
1 Will Nichols

Pacific Northwest Laboratory

P.O. Bex 999

M/S K6-77

Richland, WA 99352

1 M. D. White

Pacific Northwest Laboratory

P.O. Box 999

M/S KC 77

Richland, WA 99352

1 U-S. Park

Science Applications International

Corporation

101 Convention Center Drive

Las Vegas, NV 89109

1 Chris Pflum

Science Applications International

Corporation

101 Convention Center Drive

Las Vegas, NV 89109

1 William Dudley

U.S. Geological Survey

P.O. Box 25046

$\mathrm{M} / \mathrm{S} 425$

Denver, CO 80225

1 Dwight Hoxie

U.S. Geologi al Survey

101 Convention Center Drive

Suite 860

Las Vegas, NV 89109

1 Ed Weeks

U. S. Geolopical Survey

P.O. Box 25046

Federal Center, M/S 413

Lakewood, CO 80225 


$\begin{array}{lcl}1 & 6300 & \text { D. E. Miller } \\ 1 & 6302 & \text { T. E. Blejwas } \\ 2 & 6318 & \text { R. J. Macer for } \\ & 100 / 121441 / \text { SAND89-7018/QA } \\ 1 & 6319 & \text { R. R. Richards } \\ 1 & 1502 & \text { J. C. Cummings } \\ 1 & 1511 & \text { J. S. Rottler } \\ 1 & 1511 & \text { P. L. Hopkins } \\ 1 & 1511 & \text { M. J. Martinez } \\ 1 & 1513 & \text { R. C. Dykhuizen } \\ 1 & 1513 & \text { R. R. Eaton } \\ 1 & 1513 & \text { R. D. Skocypec } \\ 1 & 6115 & \text { P. J. Hommert, Acting } \\ 1 & 6115 & \text { J. A. Fernandez } \\ 1 & 6115 & \text { R. J. Glass } \\ 1 & 6115 & \text { C. A. Rautman } \\ 1 & 6115 & \text { M. D. Siegel } \\ 1 & 6115 & \text { V.C. Tidwell } \\ 1 & 6304 & \text { J. Holmes } \\ 1 & 6312 & \text { F. W. Bingham } \\ 1 & 6312 & \text { R. W. Barnard } \\ 1 & 6312 & \text { G. E. Barr } \\ 1 & 6312 & \text { H. A. Dockery } \\ 1 & 6312 & \text { E. Dunn } \\ 1 & 6312 & \text { J. H. Gauthier } \\ 2 & 6312 & \text { P. G. Kaplan } \\ 1 & 6312 & \text { T. H. Robey } \\ 1 & 6312 & \text { L. H. Skinner } \\ 1 & 6312 & \text { M. L. Wilson } \\ 1 & 6313 & \text { L. S. Costin } \\ 1 & 6313 & \text { M. E. Fewell } \\ 1 & 6313 & \text { E. E. Ryder } \\ 1 & 6313 & \text { S. R. Sobolik } \\ 1 & 6513 & \text { A. H. Treadway } \\ 1 & 6900 & \text { T. O. Hunter } \\ 5 & 7141 & \text { S. A. Landenberger } \\ 3 & 7151 & \text { G. C. Claycomb } \\ 8 & 7613-2 & \text { Document Processing } \\ 1 & 65323-2 & \text { Central Technical Files } \\ 1 & 7723 & \text { F. C. Lauffer } \\ & 6341 & \text { WMT Library } \\ & 6410 & \text { D. A. Dahlgren } \\ & & \end{array}$



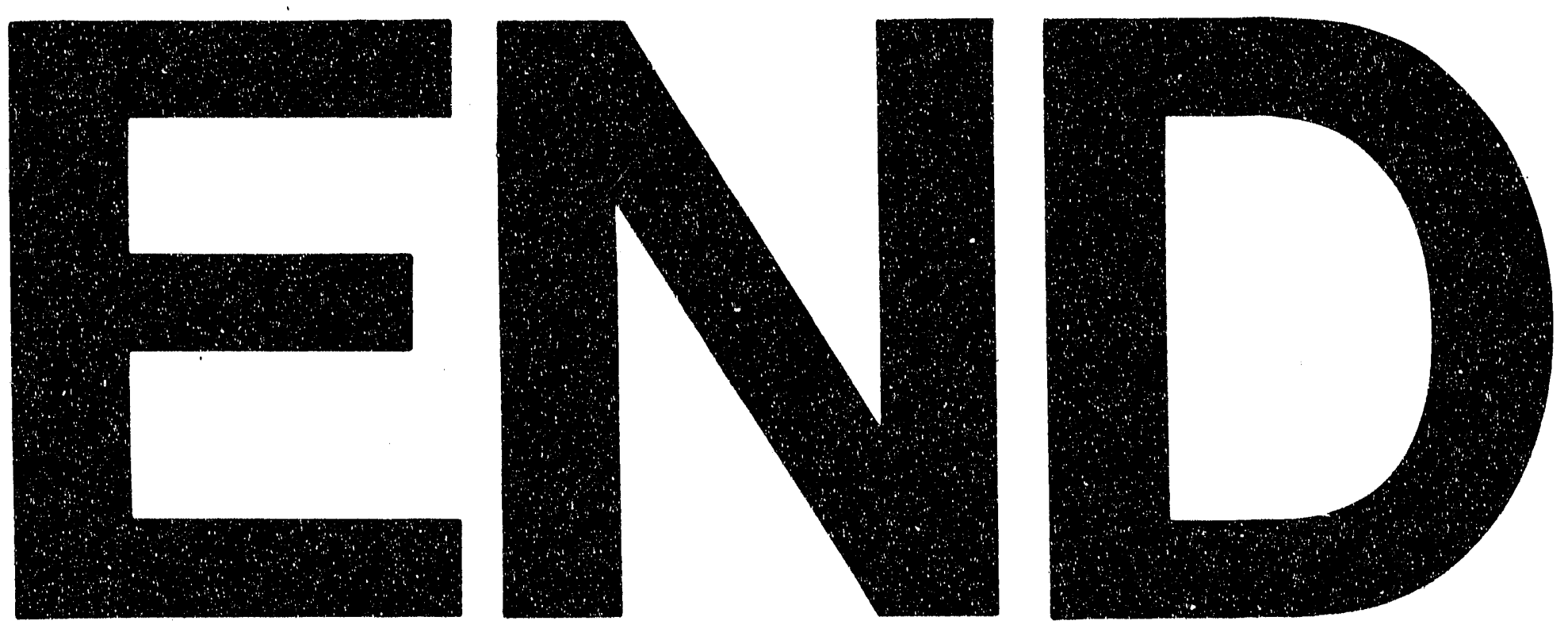

$\Rightarrow$
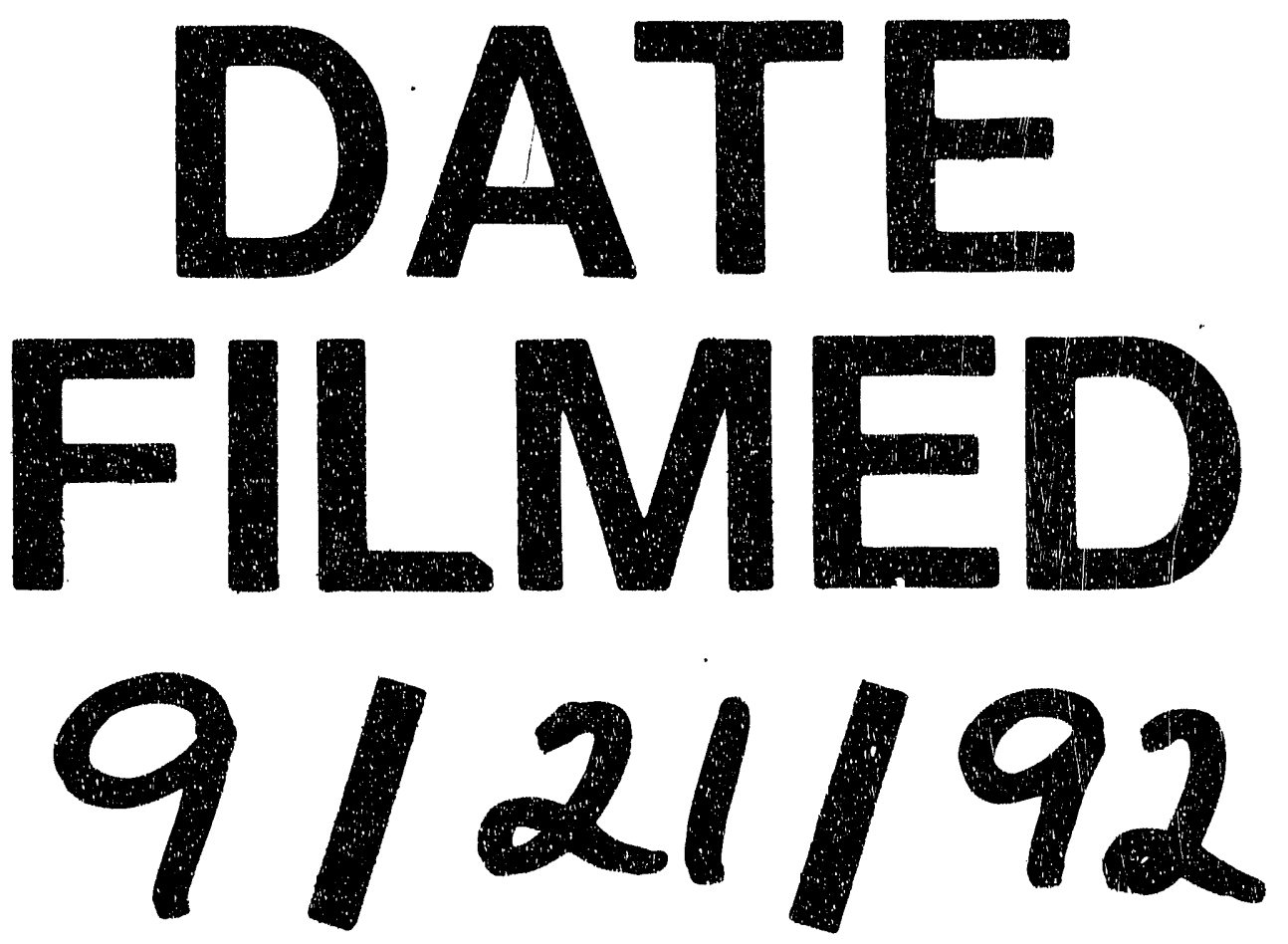
動 